\title{
Circuitos Hamiltonianos em Hipergrafos e Densidades de Subpermutações
}

\author{
Antonio Josefran de Oliveira Bastos
}

\author{
TESE APRESENTADA \\ AO \\ Instituto De Matemática e EstatísticA \\ DA \\ Universidade de São Paulo \\ PARA \\ OBTENÇÃ̃ DO TÍTULO \\ $\mathrm{DE}$ \\ DOUTOR EM CIÊNCIAS \\ Programa: Ciência da Computação \\ Orientador: Prof. Dr. Guilherme Oliveira Mota
}

Durante o desenvolvimento deste trabalho o autor recebeu auxílio financeiro da CAPES

São Paulo, agosto de 2016 


\section{Circuitos Hamiltonianos em Hipergrafos e Densidades de Subpermutações}

Esta versão da dissertação/tese contém as correções e alterações sugeridas pela Comissão Julgadora durante a defesa da versão original do trabalho, realizada em 26/08/2016. Uma cópia da versão original está disponível no Instituto de Matemática e Estatística da Universidade de São Paulo.

Comissão Julgadora:

- Prof. Dr. Guilherme Oliveira Mota (orientador) - IME-USP

- Prof. Dr. Yoshiharu Kohayakawa - USP

- Prof. Dr. Carlos Hoppen - UFRGS

- Prof. Dr. Fabrício Siqueira - UFC

- Prof. Dr. Daniel Morgato Martin - UFABC 


\section{Agradecimentos}

Inicialmente, gostaria de agradecer à minha família por todo apoio que me forneceu ao longo desse caminho, por sempre estar ao meu lado e nunca me deixar desanimar. Em especial, gostaria de agradecer profundamente à "Dona Neide" (minha mãe) e ao "Seu Bastos" (meu pai), pois eles possuem uma história inspiradora de superação e de vida sem igual. Mesmo vindos de uma terra maltratada pela seca e miséria, nunca permitiram que nos faltasse nada e sempre priorizaram nossos estudos acima de tudo.

Gostaria também de agradecer a todos os amigos com quem tive o prazer de conviver ao longo desses quatro anos que passei em São Paulo. Assim, a seguir ressalto alguns deles.

- Guilherme Mota, que sempre se preocupou comigo como um irmão e que se tornou meu orientador. A ele eu dedico um agradecimento especial, pois sempre esteve ao meu lado, me direcionando a me tornar um pesquisador e uma pessoa cada vez melhor.

- Roberto Parente, outro irmão que tive em São Paulo que me ajudou a manter a cabeça no lugar em diversos momentos e a amadurecer em diversos aspectos.

- Suelen Pereira, minha amiga, confidente e conselheira, não seria possível listar aqui todas as razões pelas quais sou grato, mas gostaria de agradecer aqui por todas as vezes em que me ajudou a superar momentos nos quais a ansiedade me cegava e por me incentivar a sempre continuar em frente. E também por ter tido a infinita paciência de revisar a minha tese.

- Nathália Yamazaki, pelas lágrimas de tristeza derramadas quando as coisas não deram certo, pelas lágrimas de felicidades derramadas quando as coisas deram certo. Por sempre se preocupar comigo, estar ao meu lado e fazer o possível para que tudo esteja bem.

- Leonardo Nagami, um amigo para a vida e um parceiro de pesquisa que participou do desenvolvimento de parte deste trabalho.

- Maria Angela, mãe da Nathália e minha segunda mãe, que, como toda mãe, sempre esteve ali. Não possuo palavras para descrever o carinho e apoio que recebi.

- Patrícia Luzmarina, uma das primeiras grandes amigas que fiz aqui em São Paulo e por quem possuo um carinho sem tamanho.

Infelizmente, os nomes de algumas grandes pessoas que apareceram ao longo dessa jornada pode me fugir à mente neste momento, mas gostaria de agradecer ainda outros grandes amigos: Rosânia Pereira, Mônica Carranza, Luiz Zambrano e Marcio Oshiro, aos meus companheiros do LOCA, Rafael Coelho, Fábio Botler e Aritanan, aos meus grandes amigos de Hamburgo, Jackob Schnitzer, Fabian Schulenbug, Mathias Schacht e Luisa Valle, aos meus amigos do grupo ParGO em Fortaleza, Rudini Menezes, Fabrício Benevides, Manoel Câmpelo e todos os outros, e a Lucileide Rodrigues pela paciência em me receber diversas vezes na secretaria e sempre fazer o possível para me ajudar.

Para finalizar, gostaria de dedicar um agradecimento especial ao Professor Yoshiharu Kohayakawa pela orientação realizada nos primeiros anos de doutorado. Além de ser uma pessoa inspiradora, graças a sua orientação pude usufruir de diversas oportunidades e absorver experiências 
diversificadas, que foram muito importantes para meu desenvolvimento como pesquisador (tais como a escola de verão no Chile e a oportunidade de Doutorado Sanduíche na Alemanha). 


\section{Resumo}

BASTOS, A. J. de O. Circuitos Hamiltonianos em Hipergrafos e Densidades de Subpermutações. 2016. Tese Doutorado - Instituto de Matemática e Estatística, Universidade de São Paulo, São Paulo, 2016.

O estudo do comportamento assintótico de densidades de algumas subestruturas é uma das principais áreas de estudos em combinatória. Na Teoria das Permutações, fixadas permutações $\tau_{1}$ e $\tau_{2}$ e um inteiro $n>0$, estamos interessados em estudar o comportamento das densidades de $\tau_{1}$ e $\tau_{2}$ na família de permutações de tamanho $n$. Assim, existem duas direções naturais que podemos seguir. Na primeira direção, estamos interessados em achar a permutação de tamanho $n$ que maximiza a densidade das permutações $\tau_{1}$ e $\tau_{2}$ simultaneamente. Para $n$ suficientemente grande, explicitamos a densidade máxima que uma família de permutações podem assumir dentre todas as permutações de tamanho $n$. Na segunda direção, estamos interessados em achar a permutação de tamanho $n$ que minimiza a densidade de $\tau_{1}$ e $\tau_{2}$ simultaneamente. Quando $\tau_{1}$ é a permutação identidade com $k$ elementos e $\tau_{2}$ é a permutação reversa com $\ell$ elementos, Myers conjecturou que o mínimo é atingido quando tomamos o mínimo dentre as permutações que não possuem a ocorrência de $\tau_{1}$ ou $\tau_{2}$. Mostramos que se restringirmos o espaço de busca somente ao conjunto de permutações em camadas, então a Conjectura de Myers é verdadeira. Por outro lado, na Teoria dos Grafos, o problema de encontrar um circuito Hamiltoniano é um problema NP-completo clássico e está entre os 21 problemas Karp. Dessa forma, uma abordagem comum na literatura para atacar esse problema é encontrar condições que um grafo deve satisfazer e que garantem a existência de um circuito Hamiltoniano em tal grafo. O célebre resultado de Dirac afirma que se um grafo $G$ de ordem $n$ possui grau mínimo pelo menos $n / 2$, então $G$ possui um circuito Hamiltoniano. Seguindo a linha de Dirac, mostramos que, dados inteiros $1 \leqslant \ell \leqslant k / 2$ e $\gamma>0$ existe um inteiro $n_{0}>0$ tal que, se um hipergrafo $k$-uniforme $\mathcal{H}$ de ordem $n$ satisfaz $\delta_{k-2}(\mathcal{H}) \geqslant\left((4(k-\ell)-1) /\left(4(k-\ell)^{2}\right)+\gamma\right)\left(\begin{array}{l}n \\ 2\end{array}\right)$, então $\mathcal{H}$ possui um $\ell$-circuito Hamiltoniano.

Palavras-chave: Circuitos Hamiltonianos, permutações, hipergrafos, combinatória assintótica, combinatória extremal. 


\section{Abstract}

BASTOS, A. J. de O. Hamiltonian Cycles in Hypergraphs and Subpermutation Densities. 2016. PhD's Thesis - Instituto de Matemática e Estatística, Universidade de São Paulo, São Paulo, 2016.

The study of asymptotic behavior of densities of some substructures is one of the main areas in combinatorics. In Permutation Theory, fixed permutations $\tau_{1}$ and $\tau_{2}$ and an integer $n>0$, we are interested in the behavior of densities of $\tau_{1}$ and $\tau_{2}$ among the permutations of size $n$. Thus, there are two natural directions we can follow. In the first direction, we are interested in finding the permutation of size $n$ that maximizes the density of the permutations $\tau_{1}$ and $\tau_{2}$ simultaneously. We explicit the maximum density of a family of permutations between all the permutations of size $n$. In the second direction, we are interested in finding the permutation of size $n$ that minimizes the density of $\tau_{1}$ and $\tau_{2}$ simultaneously. When $\tau_{1}$ is the identity permutation with $\ell$ elements and $\tau_{2}$ is the reverse permutation with $k$ elements, Myers conjectured that the minimum is achieved when we take the minimum among the permutations which do not have the occurrence of $\tau_{1}$ or $\tau_{2}$. We show that if we restrict the search space only to set of layered permutations and $k \geqslant \ell$, then the Myers' Conjecture is true. On the other hand, in Graph Theory, the problem of finding a Hamiltonian cycle is a NP-complete problem and it is among the 21 Karp problems. Thus, one approach to attack this problem is to find conditions that a graph must meet to ensure the existence of a Hamiltonian cycle on it. The celebrated result of Dirac shows that a graph $G$ of order $n$ that has minimum degree at least $n / 2$ has a Hamiltonian cycle. Following the line of Dirac, we show that give integers $1 \leqslant \ell \leqslant k / 2$ and gamma $>0$ there is an integer $n_{0}>0$ such that if a hypergraph $k$-Uniform $\mathcal{H}$ of order $n$ satisfies $\delta_{k-2}(\mathcal{H}) \geqslant\left((4(k-\ell)-1) /\left(4(k-\ell)^{2}\right)+\gamma\right)\left(\begin{array}{l}n \\ 2\end{array}\right)$, then $\mathcal{H}$ has a Hamiltonian $\ell$-cycle.

Keywords: Hamiltonian Cycle, permutation, hypergraphs, asymptotic combinatorial, extremal combinatorial. 


\section{Sumário}

1 Introdução $\quad 1$

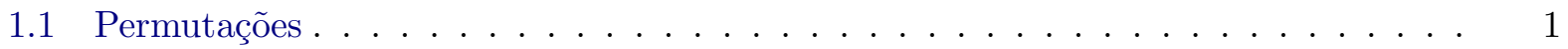

1.2 Circuitos Hamiltonianos . . . . . . . . . . . . . . . . . . 3

2 Conceitos Preliminares $\quad 5$

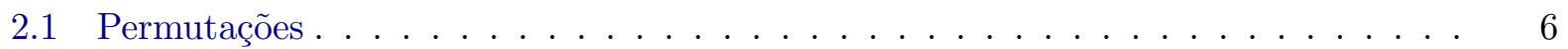

2.1 .1 Definições Básicas . . . . . . . . . . . . . . . . . 6

2.1.2 Algoritmo de Price . . . . . . . . . . . . . . . . . . . . . . 9

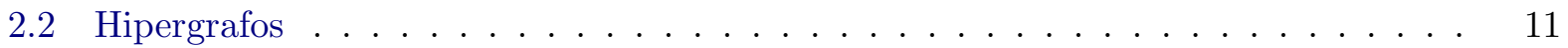

2.2 .1 Definições Básicas . . . . . . . . . . . . . . . . . . . 11

2.2.2 Regularidade Fraca em Hipergrafos . . . . . . . . . . . . . . . . . . . . . 12

3 Resultados em Permutações $\quad 15$

3.1 Problema de Empacotamento em Permutações . . . . . . . . . . . . . . . . . 17

3.1 .1 Adaptação do Algoritmo de Price . . . . . . . . . . . . . . . . . . 17

3.1.2 Resultado sobre o Problema de Empacotamento . . . . . . . . . . . . . 19

3.2 Problema de Minimização de Subsequências Monótonas . . . . . . . . . . . . . . . 28

4 Circuitos Hamiltonianos $\quad 33$

4.1 Ideia da Prova do Teorema Principal de Hipergrafos e Lemas Principais . . . . . . 35

4.1 .1 Conexão e Reservatório . . . . . . . . . . . . . . . . . . . . . 36

$4.1 .2 \quad$ Absorção . . . . . . . . . . . . . . . . . . . . . 40

4.1.3 Empacotamento dos Vértices de $\mathcal{H} \ldots \ldots \ldots$. . . . . . . . . . . 44

4.2 Prova do Teorema Principal de Hipergrafos . . . . . . . . . . . . . . . . . . . 53

5 Considerações Finais $\quad 55$

$\begin{array}{ll}\text { Referências Bibliográficas } & 57\end{array}$ 


\section{Capítulo 1}

\section{Introdução}

Abordamos três problemas clássicos em combinatória. Os dois primeiros fazem parte da Teoria das Permutações e dizem respeito ao comportamento assintótico da função densidade de uma família de permutações. Em particular, dado um inteiro positivo $n$, o primeiro problema estuda a densidade máxima que uma família de permutações pode assumir dentre todas as permutações que possuem tamanho $n$, enquanto o segundo problema estuda a densidade mínima que uma família de permutações pode assumir dentre todas as permutações de tamanho $n$. O terceiro problema abordado é um problema da Teoria dos Hipergrafos e consiste em encontrar condições que um hipergrafo deve satisfazer e que garantam a existência de um circuito Hamiltoniano.

\subsection{Permutações}

Imagine o seguinte jogo: considere um baralho de um único naipe com 13 cartas. Inicialmente escolhemos ordenadamente três cartas arbitrárias, digamos 3, 4 e 2, e as recolocamos no baralho. Agora temos que ordenar as 13 cartas do baralho de forma a maximizar o número de ocorrências do padrão (342), i.e., gostaríamos de maximizar o número de triplas de cartas do baralho que, ao considerarmos a ordem escolhida para o baralho, a primeira carta seja menor que a segunda e maior que a terceira, e que a segunda carta seja maior que a terceira. Nossa pontuação seria calculada como sendo a quantidade de repetições do padrão (342) na ordem escolhida para o baralho.

Suponha que o baralho tenha $n>0$ cartas. Analisando o jogo descrito acima, algumas das questões naturais que surgem são: dado um padrão inicial $\tau$ fixo, qual a maior pontuação possível que podemos obter? Dada uma coleção de padrões iniciais, qual a menor pontuação possível que podemos obter? Note que a segunda questão se torna trivial se possuirmos somente um padrão, pois sempre podemos ordenar as cartas de forma crescente ou decrescente e o padrão não acontecerá nenhuma vez em pelo menos uma dessas ordenações. Estudamos neste trabalho as duas questões acima voltadas para a Teoria das Permutações, i.e., estudamos o problema de maximizar e minimizar o número de ocorrências de permutações pequenas (ou padrões) em permutações maiores.

Permutações podem ser descritas como uma ordem que impomos a uma coleção de objetos utilizando algumas de suas características. Por exemplo, podemos ordenar uma caixa de lápis de cor utilizando suas tonalidades, i.e., podemos ordenar da mais clara para mais escura ou da mais escura para a mais clara. Note que, se tivermos duas caixas de lápis de cor com cores diferentes em cada caixa, podemos utilizar a mesma permutação para organizar as duas caixas (i.e., podemos 
ordenar cada uma das duas caixas colocando primeiro as cores mais claras e então as cores mais escuras). Assim, dizemos que duas permutações são equivalentes se elas definem o mesmo padrão. Por exemplo, a permutação (324) é equivalente à permutação (213). Definimos a densidade de uma permutação $\tau$, com $k$ elementos, em uma permutação $\sigma$, com $n \geqslant k$ elementos, como sendo a probabilidade de ao sortearmos uniformemente ao acaso $k$ elementos em $\sigma$ e tais elementos induzam uma permutação equivalente à permutação $\tau$. Denotamos por $p(\tau, \sigma)$ essa probabilidade. Dessa forma, as questões feitas anteriormente podem ser reformuladas como segue: dadas permutações $\tau_{1}$ e $\tau_{2}$, qual a permutação $\sigma$ de tamanho $n>0$ tal que $p\left(\tau_{1}, \sigma\right)$ é máximo? E qual a permutação $\sigma$ de tamanho $n>0$ que minimiza $p\left(\tau_{1}, \sigma\right)+p\left(\tau_{2}, \sigma\right)$ ?

A primeira questão acima é conhecida como o problema de empacotamento. Em particular, dado um inteiro $n>0$, o problema de empacotamento estuda os valores de

$$
p_{n}(\tau)=\max \{p(\tau, \sigma): \sigma \text { é uma permutação com } n \text { elementos }\}
$$

e $p(\tau)=\lim _{n \rightarrow \infty} p_{n}(\tau)$. Apesar da questão parecer simples, esse problema se mostrou extremamente desafiador. Por exemplo, existem permutações $\tau$ de tamanho 4, como a permutação (1324), para as quais $p(\tau)$ ainda é desconhecido (ver Tabela 1.1). Uma forma comum de atacar problemas em combinatória é encontrar famílias de subestruturas que nos forneçam uma intuição melhor do problema.

\begin{tabular}{|c|c|c|}
\hline Permutações $(\tau)$ & $p(\tau)$ & Referências \\
\hline$(1234),(4321)$ & 1 & trivial \\
\hline$(1432),(2341),(3214),(4123)$ & $\left(\right.$ raiz de $\left.x^{3}-12 x^{2}+156 x-64\right) \approx 0.42357$ & {$[21]$} \\
\hline$(2143),(3142)$ & $3 / 8$ & {$[21]$} \\
\hline$(1243),(2134),(3421),(4312)$ & $3 / 8$ & {$[1]$} \\
\hline$(1324),(4231)$ & $0.245 \geqslant p(\cdot) \geqslant 0.244$ & {$[21]$} \\
\hline $\begin{array}{c}(1342),(1423),(2314),(2431), \\
(3124),(3241),(4132),(4213)\end{array}$ & $0.19657 \leqslant p(1342) \leqslant 2 / 9$ & {$[1]$} \\
\hline$(2413),(3412)$ & $51 / 511 \leqslant p(2413) \leqslant 2 / 9$ & {$[1]$} \\
\hline
\end{tabular}

Tabela 1.1: Valor de Empacotamento para permutações de tamanho 4 considerando o conjunto de simetrias.

Uma permutação em camadas é uma permutação composta por uma sequência crescente de sequências decrescentes, i.e., é um permutação composta por sequências decrescentes $\left(S_{1} \cdots S_{n}\right)$ tal que para todos $1 \leqslant i<j \leqslant n$ temos que se $x \in S_{i}$ e $y \in S_{j}$ então $x<y$. Por exemplo, a permutação (1324) é uma permutação em camadas onde a sequência crescente é composta pelas subsequências decrescentes 1, 32 e 4. Albert, Atkinson, Handley, Holton e Stromquist [1] mostraram que, dada uma permutação em camadas $\tau$, para todo inteiro positivo $n$, existe uma permutação em camadas $\sigma$ de tamanho $n$ tal que $p_{n}(\tau)=p(\tau, \sigma)$. Utilizando esse resultado, Price [21] desenvolveu um ferramental para abordar o problema de empacotamento restrito às permutações em camadas. Vários trabalhos foram realizados utilizando essas ferramentas e foi possível calcular $p(\cdot)$ para diversas classes de permutações em camadas (ver $[1,12,21,27]$ ).

Uma das principais características da técnica desenvolvida por Price [21] é que ele considera apenas as subsequências decrescentes de uma permutação em camadas $\tau$ para calcular cotas para o problema de empacotamento relacionado a $\tau$. Se a permutação possuir uma sequência crescente, a técnica irá considerar isso como várias sequências decrescentes e isso irá gerar algumas complicações 
quanto às manipulações dos elementos utilizados pela técnica. Adaptamos as técnicas desenvolvidas por Price para que seja mais simples trabalhar com permutações em camadas que começam com várias sequências decrescentes de tamanho 1 consecutivas, i.e., uma sequência crescente contínua. Mais ainda, calculamos o valor de $p(\tau)$ para o caso em que $\tau$ é uma permutação em camadas que possui uma única sequência crescente (i.e., uma sequência contínua de subsequências de tamanho 1) e todas as demais subsequências decrescentes possuem pelo menos 2 elementos.

Sejam $\operatorname{Id}_{k}=12 \cdots k$ a permutação identidade e $\operatorname{Rev}_{\ell}=\ell(\ell-1) \cdots 21$ a permutação reversa. $\mathrm{O}$ problema de minimizar o número de ocorrências de certas permutações, foi amplamente estudado para minimizar $\operatorname{Id}_{k}$ e $\operatorname{Rev}_{\ell}$ (ver [2, 25]). Myers [19] apresentou a seguinte conjectura.

Conjectura 1.1. Sejam $k$ e $\ell$ inteiros positivos. Para todo inteiro $n>0$ existe uma permutação $\sigma$ de tamanho $n$ que minimiza a quantidade de ocorrências de $\operatorname{Id}_{k}$ e $\operatorname{Rev}_{\ell}$ e que possui a propriedade de que $p\left(\operatorname{Id}_{k}, \sigma\right)=0$ ou $p\left(\operatorname{Rev}_{\ell}, \sigma\right)=0$.

No resultado apresentado nesta tese no Capítulo 3, mostramos que se $k \leqslant \ell$ e o espaço de busca é restrito ao conjunto de permutações em camadas, então a conjectura proposta por Myers para $\mathrm{Id}_{k}$ e $\operatorname{Rev}_{\ell}$ é verdadeira.

\subsection{Circuitos Hamiltonianos}

Suponha que temos uma rede de computadores, onde cada computador não está necessariamente conectado a todos os outros computadores da rede, e queremos saber se é possível enviar um pacote a partir de algum computador, de tal forma que o pacote percorra toda a rede e volte ao computador inicial sem passar duas vezes pelo mesmo computador. Uma abordagem desse problema consiste em modelar a rede como um grafo $G=(V, E)$, onde cada vértice representa um computador e dois vértices estão conectados por uma aresta se e somente se os dois computadores correspondentes estão conectados. Dessa forma, o problema acima corresponde a encontrar um circuito em $G$ que passa por todos os vértices de $G$ uma única vez. Tal circuito é chamado de circuito Hamiltoniano.

O problema de encontrar um circuito Hamiltoniano em um grafo é NP-completo e faz parte dos 21 problemas de Karp [13]. Grande parte dos esforços da comunidade foram direcionados a encontrar condições e suficientes que um grafo $G=(V, E)$ deve satisfazer para que garanta a existência um circuito Hamiltoniano. Em particular, quais condições os graus dos vértices de $G$ devem satisfazer para garantir a existência de um circuito Hamiltoniano em $G$ ? Dirac [7] mostrou que se $G$ possui grau mínimo pelo menos $|V| / 2$ então $G$ possui um circuito Hamiltoniano. Ore [20] mostrou que se a soma dos graus de quaisquer dois vértices não adjacentes em $G$ é pelo menos $|V|$ então $G$ possui um circuito Hamiltoniano.

O conceito de hipergrafo $\mathcal{H}$ é a generalização de um grafo no sentido que é permitido que as arestas conectem mais de dois vértices. Um hipergrafo é dito $k$-uniforme se todas as suas arestas conectam exatamente $k$ vértices. Rödl, Ruciński e Szemerédi [23] apresentaram a seguinte generalização de circuitos para hipergrafos: dados inteiros $k \geqslant \ell>0$, um $\ell$-circuito $C_{\ell, k}(m)$ é um hipergrafo $k$-uniforme com $m$ arestas tal que existe uma ordenação cíclica dos vértices de $C_{\ell, k}(m)$ na qual toda aresta de $C_{\ell, k}(m)$ é composta por $k$ vértices consecutivos e todo par de arestas consecutivas possui exatamente $\ell$ vértices em comum (ver Figura 1.1). Em particular, um $\ell$-circuito é dito Hamiltoniano se ele contém todos os vértices do hipergrafo. Seja $A$ um subconjunto de vértices de $\mathcal{H}$. Denotamos por $\operatorname{deg}(A)$ a quantidade de arestas de $\mathcal{H}$ que contêm $A$, e.g., quando 
$\mathcal{H}$ é um hipergrafo 2-uniforme e $|A|=1$, então $\operatorname{deg}(A)$ representa a noção usual de grau em grafos.

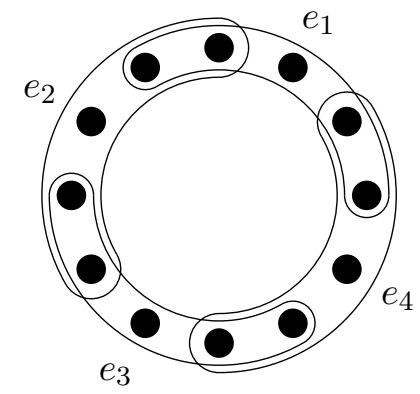

Figura 1.1: 2-circuito $C_{2,5}(4)$

Vários estudos foram realizados para tentar encontrar condições sobre o hipergrafo $\mathcal{H}$ de tal forma que $\mathcal{H}$ contenha um $\ell$-circuito Hamiltoniano (ver [5, 9, 23]). Utilizamos a técnica de absorção, desenvolvida por Rödl, Ruciński e Szemerédi [23], para mostrar que, para todo $\gamma>0$ e inteiros $k \geqslant \ell \geqslant 1$, com $k \geqslant 4$, existe um inteiro $n_{0}$ tal que todo hipergrafo $k$-uniforme $\mathcal{H}$ de ordem $n$, onde $n \geqslant n_{0}, n$ é múltiplo de $(k-\ell)$ e

$$
\operatorname{deg}(A) \geqslant\left(\frac{4(k-\ell)-1}{4(k-\ell)^{2}}+\gamma\right)\left(\begin{array}{l}
n \\
2
\end{array}\right)
$$

para todo $A \subseteq V \operatorname{com}|A|=k-2$, possui um $\ell$-circuito Hamiltoniano.

Esta tese está organizada da seguinte forma: no Capítulo 2, introduzimos os conceitos e notações que serão utilizadas ao longo deste trabalho. No Capítulo 3, apresentamos os resultados sobre permutações. O resultado obtido sobre hipergrafos é discutido no Capítulo 4. Finalmente, no Capítulo 5, fazemos alguns comentários finais. 


\section{Capítulo 2}

\section{Conceitos Preliminares}

Neste capítulo apresentamos as notações e conceitos básicos que serão utilizados ao longo desta tese. Denotamos por $[n]$ o conjunto $\{1, \ldots, n\}$. Dados um conjunto $A$ e um inteiro $k \leqslant|A|$, denotamos por $\left(\begin{array}{l}A \\ k\end{array}\right)$ o conjunto de todos os subconjuntos de $A$ que possuem exatamente $k$ elementos. Sejam $B \subseteq \mathbb{R}$ e $c \in \mathbb{R}$, representamos por $c B$ o conjunto de todos os elementos da forma $c x$, onde $x \in B$.

Sejam $k>0$ um inteiro positivo e $x, y \in \mathbb{R}_{+}^{k}$ vetores. Denotamos por $x^{y}$ o produtório $\prod_{i=1}^{k} x_{i}^{y_{i}}$. Utilizaremos ao longo deste texto o seguinte resultado, que pode ser provado utilizando indução simples em $k$.

Lema 2.1. Sejam $k>0$ um inteiro positivo e $x, y \in \mathbb{R}^{k}$ vetores com coordenadas não negativas e ordenadas de forma não decrescente. Para todo vetor $z$ gerado através de uma permutações das coordenadas de $y$, temos

$$
x^{z} \leqslant x^{y}
$$

Ao longo do Capítulo 4 será necessário utilizar o seguinte resultado.

Teorema 2.2 (Desigualdade de Chernoff). Sejam $n>0$ um inteiro positivo e $0<p \leq 1$ fixo. Sejam $X$ uma variável aleatória com distribuição binomial, $\operatorname{Bi}(n, p)$, e $\lambda=n p$. Assim, para todo $t \geq 0$, temos que

$$
\begin{gathered}
\mathbb{P}(X \geq \mathbb{E}(X)+t) \leqslant \exp \left(-\frac{t^{2}}{2(\lambda+t / 3)}\right), \\
\mathbb{P}(X \leq \mathbb{E}(X)-t) \leqslant \exp \left(-\frac{t^{2}}{2 \lambda}\right) .
\end{gathered}
$$

Em alguns momentos não precisaremos de toda a força da Desigualdade de Chernoff, de modo que a Desigualdade de Markov será suficiente.

Teorema 2.3 (Desigualdade de Markov). Seja $X$ uma variável aleatória não negativa. Para todo $t>0$, temos

$$
\mathbb{P}(X \geq t) \leqslant \frac{\mathbb{E}(X)}{t} .
$$

No Capítulo 3, calcularemos o ponto de máximo de alguns polinômios. Para isso, utilizaremos o método dos Multiplicadores de Lagrange, que é caracterizado pelo Teorema 2.4 abaixo.

Teorema 2.4. Sejam $U \subset \mathbb{R}^{n}$ um conjunto aberto e $F, G_{1}, \ldots, G_{k}: U \rightarrow \mathbb{R}$ funções com derivadas 
parciais contínuas. Defina

$$
D=\left\{x \in U: \forall i \in[k], G_{i}(x)=0\right\} .
$$

Se $y \in D$ é tal que $F(y) \geqslant F(x)$ para todo $x \in D$, então $\nabla F(y)$ pode ser escrito como um combinação linear de $\nabla G_{1}(y), \ldots, \nabla G_{k}(y)$.

A seguir apresentamos, respectivamente, alguns conceitos sobre permutações e grafos.

\subsection{Permutações}

Esta seção está dividida em duas partes. Na Subseção 2.1.1 discutimos algumas noções básicas e apresentamos algumas formas de representar permutações. Na Subseção 2.1.2 apresentamos o Algoritmo de Price.

\subsubsection{Definições Básicas}

Sejam $n>0$ um inteiro positivo e $P$ um conjunto de tamanho $n$. Uma permutação $\tau$ de $P$ é uma função bijetiva $\tau:[n] \rightarrow P$. Em outras palavras, podemos ver $\tau$ como uma ordenação dos elementos de $P$, onde o primeiro elemento é $\tau(1)$, o segundo é $\tau(2)$ e assim sucessivamente. Em particular, dizemos que $\tau$ tem tamanho $n$ e denotamos o seu tamanho por $|\tau|=n$. Por simplicidade, assumimos no restante deste texto que o conjunto $P$ é um subconjunto dos naturais.

Note que cada ordenação que definimos sobre o conjunto $P$ pode ser interpretada como uma permutação. Dessa forma, é comum representar a permutação $\tau$ apenas explicitando a ordenação definida em $P$ por $\tau$ na forma $(\tau(1) \tau(2) \ldots \tau(n))$. Por exemplo, seja $\tau$ uma permutação de $P=[4]$ tal que $\tau(1)=3, \tau(2)=1, \tau(3)=2$ e $\tau(4)=4$. Assim, temos $\tau=(3124)$.

Sejam $\tau_{1}$ uma permutação de um conjunto $P_{1}$ e $\tau_{2}$ uma permutação de um conjunto $P_{2}$, com $\left|P_{1}\right|=\left|P_{2}\right|>0$. Dizemos que $\tau_{1}$ e $\tau_{2}$ são equivalentes se ambas possuem o mesmo padrão, i.e., se para todos $i, j \in\left[\left|P_{1}\right|\right]$ temos que $\tau_{1}(i) \leqslant \tau_{1}(j)$ se e somente se $\tau_{2}(i) \leqslant \tau_{2}(j)$. Por exemplo, a permutação (423) é equivalente à permutação (312), pois ambas as permutações possuem o primeiro elemento maior que os dois elementos seguintes e o segundo elemento é menor que o terceiro elemento. Utilizaremos o sinal = para denotar quando duas permutações são equivalentes, i.e., $\sigma_{1}=\sigma_{2}$ significa que a permutação $\sigma_{1}$ é equivalente à permutação $\sigma_{2}$.

Dados um inteiro $k>0$ e $A \subseteq \mathbb{R}$, denotamos por $\mathcal{K}(A, k) \subseteq A^{k}$ o conjunto de todas as $k$-uplas ordenadas de $A$ tal que suas coordenadas estão ordenadas de forma crescente, i.e.,

$$
\mathcal{K}(A, k)=\left\{v \in A^{k}: \forall i, j \in[k],(v(i)<v(j) \Leftrightarrow i<j)\right\}
$$

Em particular, quando $A=[n]^{k}$ denotamos por $\mathcal{K}(n, k)$ o conjunto $\mathcal{K}([n], k)$ e $|\mathcal{K}(A, k)|=\left(\begin{array}{c}|A| \\ k\end{array}\right)$. Denotamos ainda por $\mathfrak{S}_{n}$ o conjunto de todas as possíveis permutações sobre $[n]$, por $\mathfrak{S}=\bigcup_{n \in \mathbb{N}} \mathfrak{S}_{n}$ o conjunto de todas as permutações finitas possíveis e por $\mathbb{R S}$ o conjunto de todas as combinações lineares formais com coeficientes reais dos elementos de $\mathfrak{S}$. Definimos $\operatorname{Id}_{k}=(12 \ldots k)$ a sequência monótona crescente de tamanho $k$ (ou permutação identidade) e $\operatorname{Rev}_{k}=(k(k-1) \ldots 1$ ) a sequência monótona decrescente de tamanho $k$ (ou permutação reversa).

Sejam $v \in \mathcal{K}(n, k)$ uma $k$-upla ordenada e $\sigma \in \mathfrak{S}_{n}$ uma permutação. A subpermutação induzida $\sigma[v]$ de $\sigma$ induzida por $v$ é a única permutação em $\mathfrak{S}_{k}$ tal que $\forall i, j \in[k]$ temos $\sigma[v](i)<\sigma[v](j)$ se 
e somente se $\sigma(v(i))<\sigma(v(j))$. Por exemplo, seja $\sigma=(4213576)$ e $v \in \mathcal{K}(7,4) \operatorname{com} v=(1,2,4,7)$. Dessa forma, temos $\sigma[v]=(4236)=(3124)$.

Estamos interessados em estudar o comportamento de subpadrões de uma permutação. Assim, dados $\sigma \in \mathfrak{S}_{n}$ e $\tau \in \mathfrak{S}_{k}$, seja $\Lambda(\tau, \sigma)$ a quantidade de ocorrências da permutação $\tau$ na permutação $\sigma$, i.e.,

$$
\Lambda(\tau, \sigma)=|\{v \in \mathcal{K}(n, k): \sigma[v]=\tau\}| .
$$

Definimos ainda a densidade de $\tau$ em $\sigma$ como

$$
p(\tau, \sigma)=\frac{\Lambda(\tau, \sigma)}{\left(\begin{array}{l}
n \\
k
\end{array}\right)} .
$$

A densidade de $\tau$ em $\sigma$ pode ser vista como a probabilidade de uma subpermutação de tamanho $k$ sorteada uniformemente ao acaso em $\sigma$ ser equivalente à permutação $\tau$. Estendemos as definições de $\Lambda$ e $p$ para o conjunto $\mathbb{R S}$ considerando-as lineares na primeira coordenada. Por exemplo, dados $c_{1}, c_{2} \in \mathbb{R}$ e $\tau_{1}, \tau_{2}, \sigma \in \mathfrak{S}$ temos

$$
p\left(c_{1} \tau_{1}+c_{2} \tau_{2}, \sigma\right)=c_{1} p\left(\tau_{1}, \sigma\right)+c_{2} p\left(\tau_{2}, \sigma\right) .
$$

A Definição 2.5 abaixo introduz os principais elementos que serão estudados no Capítulo 3.

Definição 2.5. Seja $f \in \mathbb{R S}$ uma combinação linear de permutações. Para todo $n \in \mathbb{N}$, definimos

$$
p_{n}(f)=\max \left\{p(f, \sigma): \sigma \in \mathfrak{S}_{n}\right\} \quad \text { e } \quad p(f)=\lim _{n \rightarrow \infty} p_{n}(f) .
$$

Similarmente, definimos

$$
z_{n}(f)=\min \left\{p(f, \sigma): \sigma \in \mathfrak{S}_{n}\right\} \quad \text { e } \quad z(f)=\lim _{n \rightarrow \infty} z_{n}(f) .
$$

Denotamos ainda por

$$
\operatorname{Ext}_{n}(f)=\left\{\sigma \in \mathfrak{S}_{n}: p_{n}(f)=p(f, \sigma)\right\}
$$

o conjunto das permutações que realizam o máximo e por

$$
\operatorname{Zext}_{n}(f)=\left\{\sigma \in \mathfrak{S}_{n}: z_{n}(f)=p(f, \sigma)\right\}
$$

o conjunto das permutações que realizam o mínimo. Em particular, dentro do contexto de cada problema, nos referenciamos aos elementos de $\operatorname{Ext}_{n}$ e Zext ${ }_{n}$ como permutações extremais.

Para mostrar que os limites acima estão bem definidos, note que para todos $n>0$ e $\tau \in \mathfrak{S}_{k}$ fixos e todo $\sigma \in \operatorname{Ext}_{n}(\tau)$, pela Lei das Probabilidades Totais, temos

$$
\begin{aligned}
p_{n}(\tau) & =p(\tau, \sigma) \\
& =\sum_{v \in \mathcal{K}(n, n-1)} \frac{p(\tau, \sigma[v])}{n} \\
& \leqslant \sum_{v \in \mathcal{K}(n, n-1)} \frac{p_{n-1}(\tau)}{n} \\
& =p_{n-1}(\tau) .
\end{aligned}
$$


Logo, a sequência $\left(p_{n}(\tau)\right)_{n \in \mathbb{N}}$ é monótona limitada e, portanto, convergente. Analogamente, o limite que define $z(\tau)$ está bem definido.

Uma permutação em camadas é uma permutação composta por uma sequência crescente de sequências decrescentes, i.e., uma permutação $\tau$ de tamanho $n$ é dita em camadas se existem um inteiro positivo $j \leqslant n$ e um vetor $v \in \mathcal{K}(n+1, j+1)$, com $v(j+1)=n+1$, tais que, para todo $i \in[j]$, temos $(\tau(v(i)) \tau(v(i)+1) \ldots \tau(v(i+1)-1))=\operatorname{Rev}_{v(i+1)-v(i)}$ e, para todo $m_{1}, m_{2} \in[n]$, temos $\tau\left(m_{1}\right) \leqslant \tau\left(m_{2}\right)$ se e somente se existe $i \in[j]$ tal que $m_{1} \leqslant v(i) \leqslant m_{2}$. Em particular, as sequências decrescentes maximais de uma permutação em camadas são chamadas de camadas. Por exemplo, a permutação (321654) é uma permutação em camadas, com camadas 321 e 654, a permutação $\operatorname{Rev}_{k}$ é uma permutação em camadas composta por uma única camada de tamanho $k$ e a permutação $\operatorname{Id}_{k}$ é uma permutação em camadas composta por $k$ camadas de tamanho 1 . A Afirmação 2.6 abaixo mostra que existem poucas permutações em camadas, se comparado com o total de permutações possíveis de $[n]$.

Afirmação 2.6. Para todo $n \in \mathbb{N}^{*}$ existem $2^{n-1}$ permutações em camadas de tamanho $n$.

Demonstração. Exclusivamente nessa prova adotamos uma representação particular para as permutações em camadas. Sejam $n$ um inteiro positivo e $v \in\{0,1\}^{n}$ um vetor binário com $v(1)=1$. Podemos obter uma permutação em camadas $\tau_{v}$ induzida por $v$ onde cada camada tem início na posição $i \in[n]$ se e somente se $v(i)=1$. Logo, cada vetor $v$ representa uma permutação em camadas. Por outro lado, dada uma permutação em camadas $\tau, \operatorname{com}|\tau|=n$, podemos construir um vetor binário $v \in\{0,1\}^{n}$, onde $v(i)=1$ se e somente se $\tau$ possui uma camada inciando exatamente da posição $i \in[n]$. Assim, existem exatamente $2^{n-1}$ permutações em camadas.

O fato das permutações em camadas serem compostas por uma sequência crescente de sequências decrescentes nos permite definir uma forma diferenciada de representar essas permutações. Para isso, basta observar que toda sequência de inteiros $\left(c_{i}\right)_{i=1}^{k}, \operatorname{com} c_{i}>0$, define uma única permutação em camadas de tamanho $\sum_{i=1}^{k} c_{i}$ e com $k$ camadas, onde a primeira camada tem tamanho $c_{1}$, a segunda tem tamanho $c_{2}$ e assim sucessivamente. Assim, podemos representar uma permutação em camadas exibindo uma sequência de inteiros que representam os tamanhos de suas respectivas camadas. Essa representação é chamada de decomposição em camadas de uma permutação em camadas. Por exemplo, a permutação (321654) se decompõe nas camadas 321 e 654 e representamos a decomposição como $(321654)=(3,3)$.

Uma anticamada é uma sequência contínua maximal de camadas de tamanho 1 e uma subanticamada de uma permutação em camadas é uma sequência contínua de camadas de tamanho 1. Note que não se pode ter duas anticamadas adjacentes, porém é permitido ter várias subanticamadas adjacentes. Assim, estendemos a noção de decomposição em camadas para encapsular a noção de anticamadas e subanticamadas. Para isso, denotamos por " " o elemento da sequência que representa uma anticamada ou subanticamada. Por exemplo, temos $\operatorname{Id}_{k}=(\widehat{k})$ e $(123465)=(\widehat{4}, 2)$.

Um bloco é ou uma camada de tamanho pelo menos 2 ou uma subanticamada. Dessa forma, definimos uma decomposição em blocos de uma permutação em camadas $\tau$ como uma sequência de pares ordenados $\left(\left(\ell_{i}, \xi_{i}\right)\right)_{i \in[k]}, \operatorname{com}\left(\ell_{i}, \xi_{i}\right) \in \mathbb{N} \times\{0,1\}$, onde $\ell_{i}$ é o tamanho do $i$-ésimo bloco e $\xi_{i}=1$ se e somente se o $i$-ésimo bloco é uma subanticamada, i.e., $\left(\ell_{i}, 1\right)=\widehat{\ell}_{i}$. Note que uma decomposição em blocos não é única, pois uma anticamada pode ser subdividida em várias subanticamadas. Assim, seja $\mathcal{Q}_{\tau}$ o conjunto de todas as possíveis decomposições em blocos de uma permutação em 
camadas $\tau$. Por exemplo, temos

$$
\mathcal{Q}_{12365}=\{(\widehat{3}, 2),(\widehat{2}, \widehat{1}, 2),(\widehat{1}, \widehat{2}, 2),(\widehat{1}, \widehat{1}, \widehat{1}, 2)\} .
$$

Sejam $\mathfrak{S}_{n}^{L}$ o conjunto das permutações em camadas de $[n]$ e $\mathfrak{S}^{L}=\cup_{i=1}^{\infty} \mathfrak{S}_{i}^{L}$ o conjunto de todas as possíveis permutações em camadas. O Teorema 2.7 abaixo, mostra que, para todo inteiro $n>0$ e toda permutação em camadas $\tau$ existe uma permutação em camadas $\sigma \in \mathfrak{S}_{n}^{L}$ tal que $p_{n}(\tau)=p(\tau, \sigma)$, i.e., podemos restringir nosso espaço de busca ao conjunto de permutações em camadas. Pela Afirmação 2.6, isso torna o problema muito mais simples, pois reduz consideravelmente o espaço de busca.

Teorema 2.7 (Albert, Atkinson, Handley, Holton e Stromquist [1]). Se $f \in \mathbb{R S}^{L}$ é uma combinação linear com coeficientes não-negativos de permutações em camadas então para todo $n \in \mathbb{N}$ temos $p_{n}(f)=\max \left\{p(f, \sigma): \sigma \in \mathfrak{S}_{n}^{L}\right\}$.

Fazendo uso do Teorema 2.7 acima, Price [21] construiu um algoritmo para calcular cotas inferiores para $p_{n}(f)$. Esse algoritmo será discutido na Seção 2.1.2.

\subsubsection{Algoritmo de Price}

Sejam $\tau=(2,2)$ e $\sigma_{1}=(3,3,4)$ permutações em camadas. Para calcular a quantidade $\Lambda\left(\tau, \sigma_{1}\right)$ de ocorrências de $\tau$ em $\sigma_{1}$, note que em toda ocorrência cada camada da permutação $\tau$ deve estar inteiramente contida em uma camada da permutação $\sigma_{1}$. Portanto,

$$
\Lambda\left(\tau, \sigma_{1}\right)=\left(\begin{array}{l}
3 \\
2
\end{array}\right)\left(\begin{array}{l}
3 \\
2
\end{array}\right)+\left(\begin{array}{l}
3 \\
2
\end{array}\right)\left(\begin{array}{l}
4 \\
2
\end{array}\right)+\left(\begin{array}{l}
3 \\
2
\end{array}\right)\left(\begin{array}{l}
4 \\
2
\end{array}\right)=45 .
$$

Por outro lado, para a permutação em camadas $\sigma_{2}=(5,5)$ temos

$$
\Lambda\left(\tau, \sigma_{2}\right)=\left(\begin{array}{l}
5 \\
2
\end{array}\right)\left(\begin{array}{l}
5 \\
2
\end{array}\right)=100
$$

Apesar de $\sigma_{1}$ ter mais camadas que $\sigma_{2}$, a permutação $\tau$ ocorre mais vezes na permutação $\sigma_{2}$. Esse comportamento nos leva a pensar se existe alguma permutação de tamanho 10 que tem 3 camadas não vazias e maximiza o número de ocorrências de $\tau$. Para verificar isso, considere $\left(c_{1}, c_{2}, c_{3}\right)$ uma permutação em camadas de tamanho 10. Assim, queremos maximizar a função

$$
f\left(c_{1}, c_{2}, c_{3}\right)=\left(\begin{array}{c}
c_{1} \\
2
\end{array}\right)\left(\left(\begin{array}{c}
c_{2} \\
2
\end{array}\right)+\left(\begin{array}{c}
c_{3} \\
2
\end{array}\right)\right)+\left(\begin{array}{c}
c_{2} \\
2
\end{array}\right)\left(\begin{array}{c}
c_{3} \\
2
\end{array}\right)
$$

restrita a $c_{1}+c_{2}+c_{3}=10$. Utilizando Multiplicadores de Lagrange (ver Teorema 2.4), verificamos que os pontos de máximo da função acima são os pontos $(5,5,0),(5,0,5)$ e $(0,5,5)$, i.e., quando uma das variáveis é nula e as restantes possuem o mesmo valor.

Price [21] observou que, para algumas permutações em camadas $\tau$, existe uma constante $c_{\tau}$ tal que para todo inteiro positivo $n$ existe uma permutação em camadas $\sigma \in \operatorname{Ext}_{n}(\tau)$ que possui no máximo $c_{\tau}$ camadas. Assim, ele desenvolveu um ferramental para obter cotas para $p(\cdot)$ através de permutações com o número limitado de camadas.

Seja $\tau=\left(\ell_{1}, \ldots, \ell_{k}\right)$ uma permutação em camadas. O Polinômio de Price de ordem $n>0$ 
relacionado à permutação $\tau$ é dado por

$$
q_{n, \tau}\left(x_{1}, \ldots, x_{n}\right)=|\tau| ! \sum_{v \in \mathcal{K}(n, k)} \prod_{j=1}^{k} x_{v(j)}^{\ell_{j}} / \ell_{j} !
$$

no qual um somatório vazio retorna 0 e um produtório vazio retorna 1 . Note que o Polinômio de Price pode ser interpretado como a densidade de $\tau$ em uma permutação em camadas de tamanho "infinito" e com $n$ camadas. Estendemos linearmente o Polinômio de Price para uma combinação $f \in \mathbb{R S}^{L}, \operatorname{com} f=\sum a_{i} \tau_{i}$, por

$$
q_{n, f}=\sum a_{i} q_{n, \tau_{i}}
$$

A Cota de Price de ordem $n>0$ para $f$ é

$$
\mathcal{L}_{n, f}=\max \left\{q_{n, f}\left(x_{1}, \ldots, x_{n}\right): \sum_{j=1}^{n} x_{j}=1 \text { e } \forall j \in[n], x_{j} \geqslant 0\right\} .
$$

Por argumentos de compacidade, ressaltamos que o máximo acima existe. Seja $f \in \mathbb{R} \mathfrak{S}$ com $f=$ $a_{1} \pi_{1}+\cdots+a_{n} \pi_{n}$. Dizemos que $f$ é uma combinação linear cônica de elementos de $\mathfrak{S}$ se $a_{i} \geqslant 0$ para todo $1 \leqslant i \leqslant n$. A Proposição 2.8 abaixo mostra que a sequência $\left(\mathcal{L}_{n, f}\right)_{n \in \mathbb{N}}$ é não decrescente e converge para $p(f)$.

Proposição 2.8 (Price [21]). Se $f \in \mathbb{R S}^{L}$ é uma combinação linear cônica de permutações em camadas então

$$
\mathcal{L}_{n, f} \leqslant \mathcal{L}_{n+1, f} \leqslant p(f) .
$$

Mais ainda, temos $\lim _{n \rightarrow \infty} \mathcal{L}_{n, f}=p(f)$.

A Proposição 2.8 sugere um algoritmo, conhecido por Algoritmo de Price, para se obter cotas inferiores para $p(f)$, o qual consiste em fixar um $n>0$ e encontrar a Cota de Price para aquele $n$ e então repetir o mesmo processo para $n+1$. Em outras palavras, o algoritmo consiste em ir aumentando iterativamente o número de camadas da permutação até que o valor ótimo seja atingido. Em alguns casos, o valor ótimo é atingido para um valor fixo de $n$, que depende de $\tau$. Porém, Albert, Atkinson, Handley, Holton e Stromquist [1], mostraram que, se uma permutação $\tau$ em camadas com 3 camadas possui a primeira ou a última camada de tamanho 1 então para todo $n>0$, temos $\mathcal{L}_{n, \tau}<p(\tau)$.

Sejam $n>0$ um inteiro positivo, $x=\left(x_{1}, \ldots, x_{n}\right) \in[0,1]^{n}$ uma $n$-upla ordenada e $\tau \in \mathfrak{S}^{L}$ uma permutação em camadas, com $|\tau|<n$. Suponha que $x \in \mathcal{K}([0,1], n)$ e que $q_{n, \tau}(x)=\mathcal{L}_{n, \tau}$. Uma abordagem para mostrar que o algoritmo para em algum momento, e que será amplamente utilizada no Capítulo 3 é a seguinte: seja $n_{0}$ o valor candidato a ponto de parada do algoritmo e considere $n>n_{0}$. Assim, temos que mostrar que

$$
q_{n, \tau}\left(x_{1}, \ldots, x_{n}\right) \geqslant q_{n-1, \tau}\left(x_{1}+x_{2}, x_{3}, \ldots, x_{n}\right),
$$

i.e., se juntarmos as duas menores camadas da permutação extremal (ver Definição 2.5) em um única camada então não aumentamos o valor do Polinômio de Price. 


\subsection{Hipergrafos}

Esta seção está dividida em duas partes. Na Seção 2.2.1, discutimos algumas notações de grafos e hipergrafos, bem como alguns resultados básicos que serão utilizados ao longo do Capítulo 4 . Na Seção 2.2.2, discutimos uma generalização do Lema da Regularidade de Szemerédi para hipergrafos.

\subsubsection{Definições Básicas}

Um hipergrafo $\mathcal{H}$ de ordem $n \in \mathbb{N}$ é um par ordenado $(V(\mathcal{H}), E(\mathcal{H}))$ onde $V(\mathcal{H})$ é um conjunto com $n$ elementos e $E(\mathcal{H})$ é um conjunto de subconjuntos de $V(\mathcal{H})$. Dizemos que $V(\mathcal{H})$ é o conjunto dos vértices de $\mathcal{H}$ e $E(\mathcal{H})$ é o conjunto das arestas de $\mathcal{H}$. Por simplicidade, quando não houver risco de ambiguidade, iremos denotar apenas por $(V, E)$ o conjunto de vértices e arestas do hipergrafo $\mathcal{H}$.

Seja $0 \leqslant k \leqslant n$ um inteiro positivo. Um hipergrafo $k$-uniforme é um hipergrafo $\mathcal{H}=(V, E)$ tal que $E \subseteq\left(\begin{array}{l}V \\ k\end{array}\right)$. Denotamos por $v(\mathcal{H})$ a quantidade de vértices do hipergrafo $\mathcal{H}$ e por $e(\mathcal{H})$ a quantidade de arestas do hipergrafo $\mathcal{H}$.

Considere hipergrafos $\mathcal{H}=(V, E)$ e $\mathcal{H}^{\prime}=\left(V^{\prime}, E^{\prime}\right)$. Dizemos que $\mathcal{H}^{\prime}$ é um subhipergrafo de $\mathcal{H}$ se $V^{\prime} \subseteq V$ e $E^{\prime} \subseteq E$. Em particular, dizemos que $\mathcal{H}^{\prime}$ é um subhipergrafo induzido de $\mathcal{H}$ se $V^{\prime} \subseteq V$ e para toda aresta $e \in E$, se $e \subseteq V^{\prime}$ então $e \in E^{\prime}$. Dado $A \subseteq V$, denotamos por $\mathcal{H}[A]$ o subhipergrafo de $\mathcal{H}$ induzido por $A$, i.e., um subhipergrafo induzido de $\mathcal{H}$ com conjunto de vértices $A$.

Sejam $\mathcal{P}$ um hipergrafo $k$-uniforme e $1 \leqslant \ell \leqslant k$ um inteiro fixo. Dizemos que $\mathcal{P}$ é um $\ell$-caminho se existe uma ordenação $v_{1}, \ldots, v_{|V(\mathcal{P})|}$ dos vértices de $\mathcal{P}$ tal que toda aresta de $\mathcal{P}$ é composta por $k$ vértices consecutivos nessa ordenação e quaisquer duas arestas consecutivas se intersectam-se em exatamente $\ell$ vértices. Em particular, dizemos que $\left\{v_{1}, \ldots, v_{\ell}\right\}$ e $\left\{v_{|\mathcal{P}|-\ell+1}, \ldots, v_{|\mathcal{P}|}\right\}$ são as extremidades do $\ell$-caminho $\mathcal{P}$. Se essa ordenação for cíclica e $\left\{v_{1}, \ldots, v_{\ell}\right\}=\left\{v_{|\mathcal{P}|-\ell+1}, \ldots, v_{|\mathcal{P}|}\right\}$ então dizemos que $\mathcal{P}$ é um $\ell$-circuito. Um subhipergrafo de $\mathcal{H}$ é um $\ell$-circuito Hamiltoniano se é um $\ell$-circuito que cobre todos os vértices de $\mathcal{H}$. Uma clique ou hipergrafo completo de ordem $n>0$ é um hipergrafo $K_{n} k$-uniforme com $n$ vértices que possui todas as arestas possíveis. Dizemos que $\mathcal{H}=(V, E)$ é $m$-partido, para algum $m>0$, se existe uma família de subconjuntos $A_{1}, \ldots, A_{m} \subseteq V$ tal que o seguinte vale:

i) $V=\bigcup_{i=1}^{m} A_{i}$

ii) $A_{i} \cap A_{j}=\emptyset$ para todos $i, j \in[m] \operatorname{com} i \neq j$;

iii) $e\left(\mathcal{H}\left[A_{i}\right]\right)=0$ para todo $1 \leqslant i \leqslant m$.

Os subconjuntos $A_{1}, \ldots, A_{m}$ são chamados de partes de $\mathcal{H}$.

Dados um conjunto $A \subseteq V$, com $|A| \leqslant k$, e um inteiro $j>0$ definimos a $j$-vizinhança de $A$ no hipergrafo $k$-uniforme $\mathcal{H}$ como

$$
N_{\mathcal{H}}^{j}(A)=\left\{B \in\left(\begin{array}{c}
V \backslash A \\
j
\end{array}\right): \exists e \in E \operatorname{com} A \cup B \subset e\right\} .
$$

Para simplificar a notação, omitiremos o $j$ na notação acima quando $j=k-|A|$. Denotamos por $\operatorname{deg}(A)=\left|N_{\mathcal{H}}(A)\right|$ o grau do conjunto $A$ e por

$$
\delta_{i}(\mathcal{H})=\min _{A \in\left(\begin{array}{c}
V \\
i
\end{array}\right)} \operatorname{deg}(A)
$$


o $i$-grau mínimo do hipergrafo $\mathcal{H}$.

Um emparelhamento $M \subseteq E$ de $\mathcal{H}$ é uma coleção de arestas de $\mathcal{H}$ duas a duas disjuntas, i.e., para todas arestas $e_{1}, e_{2} \in M$, com $e_{1} \neq e_{2}$, temos que $e_{1} \cap e_{2}=\emptyset$. Dizemos que um conjunto $A \subseteq V$ é uma cobertura de $\mathcal{H}$ se para toda aresta $e \in E$ temos $e \cap A \neq \emptyset$. O Teorema 2.9 abaixo é um resultado clássico da Teoria dos Grafos que relaciona o tamanho da cobertura mínima de um grafo bipartido com o tamanho de seu emparelhamento máximo.

Teorema 2.9 (Teorema de König [16]). Se $G$ é um grafo bipartido então o tamanho da cobertura mínima de $G$ é igual ao tamanho do emparelhamento máximo de $G$.

O hipergrafo link $\mathcal{H}_{A}$ de um conjunto $A \subseteq V$ de vértices, com $0<|A|<k$, é um hipergrafo $(k-|A|)$-uniforme com vértices em $V \backslash A$ e arestas

$$
E\left(\mathcal{H}_{A}\right)=\left\{e \in\left(\begin{array}{c}
V \backslash A \\
k-|A|
\end{array}\right): e \cup A \in E\right\}
$$

\subsubsection{Regularidade Fraca em Hipergrafos}

Nesta seção, vamos apresentar o Lema de Regularidade Fraca para hipergrafos, que é uma extensão direta do Lema da Regularidade de Szemerédi para grafos [26]. Seja $\mathcal{H}=(V, E)$ um hipergrafo $k$-uniforme e sejam $V_{1}, \ldots, V_{k}$ conjuntos não vazios e mutuamente disjuntos de $V$. Denotamos a quantidade de arestas com exatamente um vértice em cada $V_{i}$ como $e_{\mathcal{H}}\left(V_{1}, \ldots, V_{k}\right)$ e definimos a densidade de arestas de $\mathcal{H}$ em $\left(V_{1}, \ldots, V_{k}\right)$ por

$$
d_{\mathcal{H}}\left(V_{1}, \ldots, V_{k}\right)=\frac{e_{\mathcal{H}}\left(V_{1}, \ldots, V_{k}\right)}{\left|V_{1}\right| \cdots\left|V_{k}\right|} .
$$

Para $\varepsilon>0$ e $d>0$, uma $k$-upla $\left(V_{1}, \ldots, V_{k}\right)$ de subconjuntos mutuamente disjuntos de vértices é chamada $(\varepsilon, d)$-regular se, para todos conjuntos $A_{1}, \ldots, A_{k}$ com $A_{i} \subseteq V_{i}$ e $\left|A_{i}\right| \geqslant \varepsilon\left|V_{i}\right|$ para $1 \leqslant i \leqslant k$, temos

$$
\left|d_{\mathcal{H}}\left(A_{1}, \ldots, A_{k}\right)-d\right| \leqslant \varepsilon .
$$

Mais ainda, $\left(V_{1}, \ldots, V_{k}\right)$ é dito $\varepsilon$-regular se é $(\varepsilon, d)$-regular para algum $d>0$. O Lema 2.10 abaixo é uma consequência do Lema da Regularidade de Szemerédi para grafos (ver [6]).

Lema 2.10 (Lema da Regularidade Fraca). Para todos inteiros $k \geqslant 2$ e $t_{0} \geqslant 1$ e para todo $\varepsilon>0$, existe $T_{0}=T_{0}\left(k, t_{0}, \varepsilon\right)$ tal que, para todo hipergrafo $k$-uniforme $\mathcal{H}=(V, E)$ de ordem $n$, onde $n$ é suficientemente grande, existe uma partição $V=V_{0} \dot{\cup} V_{1} \dot{\cup} \ldots \dot{U} V_{t}$ satisfazendo

i) $t_{0} \leqslant t \leqslant T_{0}$,

ii) $\left|V_{1}\right|=\cdots=\left|V_{t}\right|$ e $\left|V_{0}\right| \leqslant \varepsilon n$,

iii) Para pelos menos $(1-\varepsilon)\left(\begin{array}{l}t \\ k\end{array}\right)$ subconjuntos $\left\{i_{1}, \ldots, i_{k}\right\} \subset[t]$, temos que $\left(V_{i_{1}}, \ldots, V_{i_{k}}\right)$ é $\varepsilon$-regular.

Uma partição dos vértices de um hipergrafo $\mathcal{H}$ satisfazendo os itens i-iii da conclusão do Lema 2.10 acima é chamada de partição $\varepsilon$-regular. Para $\varepsilon>0$ e $d>0$, definimos o hipergrafo reduzido $\mathcal{R}=\mathcal{R}(\varepsilon, d)$ de $\mathcal{H}$ com respeito à partição $\varepsilon$-regular $V_{0} \dot{\cup} V_{1} \dot{\cup} \cdots \dot{\cup} V_{t}$ como o hipergrafo $k$-uniforme com vértices em $[t]$ tal que

$$
\left\{i_{1}, \ldots, i_{k}\right\} \in E(\mathcal{R}) \Longleftrightarrow V_{i_{1}} \dot{\cup} V_{i_{2}} \dot{\cup} \ldots \dot{\cup} V_{i_{k}} \text { é }\left(\varepsilon, d^{\prime}\right) \text {-regular, para algum } d^{\prime} \geqslant d \text {. }
$$


Em uma aplicação típica do Lema da Regularidade, o hipergrafo reduzido herda algumas características chave do hipergrafo $\mathcal{H}$. De fato, o Lema 2.11 abaixo mostra que o hipergrafo reduzido herda aproximadamente a condição de grau mínimo do hipergrafo original.

Lema 2.11. Dados $c, \varepsilon, d>0$ e inteiros $k \geqslant 3$ e $t_{0} \geqslant 2 k / d$, seja $\mathcal{H}$ um hipergrafo $k$-uniforme de ordem $n \geqslant t \geqslant t_{0}$ tal que

$$
\delta_{k-2}(\mathcal{H}) \geqslant c\left(\begin{array}{l}
n \\
2
\end{array}\right)
$$

Se $\mathcal{H}$ possui uma partição $\varepsilon$-regular $V_{0} \dot{\cup} V_{1} \dot{\cup} \ldots \dot{\cup} V_{t}$ com hipergrafo reduzido $\mathcal{R}=\mathcal{R}(\varepsilon, d)$ então pelo menos $(1-\sqrt{\varepsilon})\left(\begin{array}{c}t \\ k-2\end{array}\right)$ conjuntos $K$ com $k-2$ elementos de $[t]$ satisfazem

$$
\operatorname{deg}_{\mathcal{R}}(K) \geqslant(c-2 d-\sqrt{\varepsilon})\left(\begin{array}{l}
t \\
2
\end{array}\right)
$$

Demonstração. Sejam $\mathcal{D}=\mathcal{D}(d)$ e $\mathcal{N}=\mathcal{N}(\varepsilon)$ hipergrafos com vértices em $[t]$ onde

- $E(\mathcal{D})$ consiste em todos os conjuntos $\left\{i_{1}, \ldots, i_{k}\right\}$ tais que $d\left(V_{i_{1}}, \ldots, V_{i_{k}}\right) \geqslant d \mathrm{e}$

- $E(\mathcal{N})$ consiste em todos os conjuntos $\left\{i_{1}, \ldots, i_{k}\right\}$ tais que $\left(V_{i_{1}}, \ldots, V_{i_{k}}\right)$ não é $\varepsilon$-regular.

Note que o hipergrafo reduzido $\mathcal{R}(\varepsilon, d)$ é o hipergrafo com vértices em $[t]$ e conjunto de arestas $E(\mathcal{D}) \backslash E(\mathcal{N})$. Para um conjunto arbitrário $K=\left\{i_{1}, \ldots, i_{k-2}\right\} \in\left(\begin{array}{c}{[t]} \\ k-2\end{array}\right)$, mostraremos que

$$
\operatorname{deg}_{\mathcal{D}}(K) \geqslant(c-2 d)\left(\begin{array}{l}
t \\
2
\end{array}\right)
$$

Seja $n / t \geqslant\left|V_{i_{j}}\right|=m \geqslant(1-\varepsilon) n / t$ o tamanho das classes da partição e seja $x$ a quantidade de arestas em $\mathcal{H}$ que intersectam o conjunto $V_{i_{j}}$ em exatamente um vértice para todo $j \in[k-2]$. Pelas restrições em $\delta_{k-2}(\mathcal{H})$ e $t \geqslant t_{0} \geqslant 2 k / d$, obtemos

$$
x \geqslant m^{k-2}\left(c\left(\begin{array}{l}
n \\
2
\end{array}\right)-(k-2) m n\right) \geqslant(c-d) m^{k-2}\left(\begin{array}{l}
n \\
2
\end{array}\right) .
$$

Se (2.3) não for verdadeira então podemos limitar $x$ por

$$
x<(c-2 d)\left(\begin{array}{l}
t \\
2
\end{array}\right) m^{k}+\left(\begin{array}{l}
t \\
2
\end{array}\right) d m^{k} \leqslant(c-d) m^{k-2}\left(\begin{array}{l}
n \\
2
\end{array}\right)
$$

contradizendo a cota inferior de $x$. Portanto, (2.3) é válida.

Observe que pelo menos $(1-\sqrt{\varepsilon})\left(\begin{array}{c}t \\ k-2\end{array}\right)$ conjuntos $K$, com $|K|=k-2$, satisfazem $\operatorname{deg}_{\mathcal{N}}(K) \leqslant$ $\sqrt{\varepsilon}\left(\begin{array}{l}t \\ 2\end{array}\right)$, pois o número de $k$-uplas que não são $\varepsilon$-regulares em $\mathcal{R}$ é no máximo $\varepsilon\left(\begin{array}{l}t \\ k\end{array}\right)$. Consequentemente, pela condição de grau mínimo em $\mathcal{D}$ e $\mathcal{N}$, no mínimo $(1-\sqrt{\varepsilon})\left(\begin{array}{c}t \\ k-2\end{array}\right)$ conjuntos $K$, com $|K|=k-2$, satisfazem

$$
\operatorname{deg}_{\mathcal{R}}(K) \geqslant(c-2 d-\sqrt{\varepsilon})\left(\begin{array}{l}
t \\
2
\end{array}\right)
$$




\section{Capítulo 3}

\section{Resultados em Permutações}

O trabalho apresentado neste capítulo foi realizado em conjunto com Leonardo Nagami Coregliano e pode ser conferido em [3].

$\mathrm{Na}$ área que estuda o comportamento de padrões em permutações, muitos dos problemas podem ser categorizados em três classes que consistem em estudar a família de permutações que evitam, maximizam ou minimizam as ocorrências de uma família de padrões fixos.

O Problema 3.1 abaixo, conhecido como Problema de Empacotamento em Permutações, está relacionado com o estudo assintótico de permutações que maximizam a quantidade de ocorrências de uma família de padrões, i.e., estamos interessados em estudar a função $p(\cdot)$ (ver Definição 2.5) para famílias particulares de permutações.

Problema 3.1 (Empacotamento em Permutações). Seja $f \in \mathbb{R} \mathfrak{S}$ uma combinação linear cônica de permutações. Para todo $n \in \mathbb{N}$, qual o valor de $p_{n}(f)$ e quais são as propriedades que uma permutação $\sigma \in \operatorname{Ext}_{n}(f)$ possui?

O valor $p(f)$ é conhecido como o valor de empacotamento de $f$. Com o surgimento do Teorema 2.7, e das ferramentas desenvolvidas por Price [21], discutidas na Seção 2.1.2, mais esforços vêm sendo colocados para encontrar o valor de empacotamento para as permutações em camadas do que para permutações em geral. Albert, Atkinson, Handley, Holton e Stromquist [1] resolveram o Problema 3.1 para a família de permutações em camadas que possuem duas camadas. Hästo [12] resolveu o Problema 3.1 para qualquer permutação em camadas que possui $r$ camadas, onde cada camada possui tamanho pelo menos $\log (r+1)$. Ele ainda calculou o valor de empacotamento para permutações em camadas da forma $(k, 1, k)$ com $k \geqslant 3$. No nosso primeiro resultado, calculamos o valor de empacotamento para uma certa família de permutações que possui uma anticamada.

Teorema 3.2. Sejam $a, \ell_{1}, \ell_{2}, \ldots, \ell_{k} \in \mathbb{N}$ inteiros positivos tais que $2 \leqslant a \leqslant \ell_{1} \leqslant \cdots \leqslant \ell_{k}$ e $2^{a}-a-1 \geqslant k$. Se $\sigma$ é uma permutação em camadas da forma $\left(\widehat{a}, \ell_{1}, \ldots, \ell_{k}\right)$ então

$$
p(\sigma)=\frac{a^{a}|\sigma| !}{a !|\sigma|^{|\sigma|}} \prod_{i=1}^{k} \frac{\ell_{i}^{\ell_{i}}}{\ell_{i} !} .
$$

É natural pensar no dual do Problema 3.1, i.e., tentar encontrar permutações que minimizam a quantidade de ocorrências de permutações menores. Dessa forma, apresentamos o Problema 3.3 abaixo. 
Problema 3.3. Seja $f \in \mathbb{R S}$ uma combinação linear cônica de permutações. Para todo $n \in \mathbb{N}$, qual o valor de $z_{n}(f)$ e quais são as propriedades que uma permutação $\sigma \in \operatorname{Zext}_{n}(f)$ possui?

Seja $f \in \mathbb{R S}^{L}$ uma combinação linear de permutações em camadas. Para todo $n \in \mathbb{N}$, definimos

$$
\begin{aligned}
z_{n}^{\prime}(f) & =\min \left\{p(f, \sigma): \sigma \in \mathfrak{S}_{n}^{L}\right\} ; \\
\operatorname{Zext}_{n}^{\prime}(f) & =\left\{\sigma \in \mathfrak{S}_{n}^{L}: p(f, \sigma)=z_{n}^{\prime}(f)\right\} ; \\
z^{\prime}(f) & =\lim _{n \rightarrow \infty} z_{n}^{\prime}(f) .
\end{aligned}
$$

Note que podemos reformular o Problema 3.3 como um Problema de Empacotamento em Permutações, i.e., ao invés de minimizar uma determinada permutação podemos maximizar todas as outras. Essa abordagem seria pouco proveitosa, pois o resultado do Teorema 2.7 não poderia mais ser utilizado. Assim, o Problema 3.4 abaixo é um problema totalmente diferente do Problema 3.3.

Problema 3.4 (Minimização de Permutações em Camadas). Seja $f \in \mathbb{R S}^{L}$ uma combinação linear cônica de permutações em camadas. Para todo $n \in \mathbb{N}$, qual o valor de $z_{n}^{\prime}(f)$ e quais são as propriedades que uma permutação $\sigma \in \operatorname{Zext}_{n}^{\prime}(f)$ possui?

Erdős e Szekeres [8] mostraram que toda permutação com $k^{2}+1$ elementos deve conter uma subsequência monótona de tamanho $k+1$. Mais tarde, Myers [19] mostrou a seguinte versão quantitativa desse teorema.

Teorema 3.5 (Myers [19]). Temos

$$
z\left(\operatorname{Id}_{3}+\operatorname{Rev}_{3}\right)=\frac{1}{4}
$$

Mais ainda, para todo $k \geqslant 2$, temos

$$
\lim _{n \rightarrow \infty} \min \left\{p\left(\operatorname{Rev}_{k+1}, \sigma\right): \sigma \in \mathfrak{S}_{n} \operatorname{com} p\left(\operatorname{Id}_{k+1}, \sigma\right)=0\right\}=\frac{1}{k^{k}} .
$$

A segunda parte do Teorema 3.5 acima levou Myers a conjecturar que $z\left(\operatorname{Id}_{k+1}+\operatorname{Rev}_{k+1}\right)=1 / k^{k}$ para todo $k \geqslant 2$. Apresentamos abaixo a versão generalizada da Conjectura de Myers.

Conjectura 3.6 (Generalização da Conjectura de Myers [19]). Para todo $k, \ell \geqslant 2$, temos

$$
z\left(\operatorname{Id}_{\ell+1}+\operatorname{Rev}_{k+1}\right)=\min \left\{\frac{1}{k^{\ell}}, \frac{1}{\ell^{k}}\right\}
$$

O mínimo acima é uma cota superior para $z\left(\operatorname{Id}_{\ell+1}+\operatorname{Rev}_{k+1}\right)$, obtido considerando apenas per-

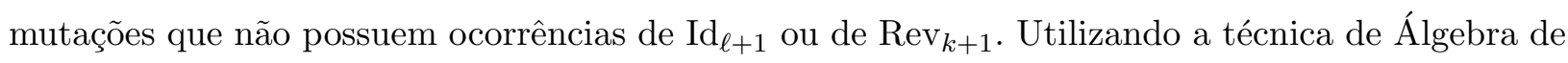
Flags, desenvolvida por Razborov [22], Balogh, Hu, Lidický, Pikhurko, Udvari e Volec [2] mostraram que a Conjectura 3.6 acima é verdadeira para $k=\ell=3$. Samotij e Sudakov [25] mostraram que a conjetura é verdadeira quando $k=\ell$ e $n \leqslant k^{2}+c k^{c / 2} \log k$, para $k$ e $c$ suficientemente grandes. Em nosso segundo resultado, mostramos que uma versão simplificada da conjectura, na qual consideramos o espaço de busca como sendo o espaço das permutações em camadas, é verdadeira, i.e., calculamos $z^{\prime}\left(\operatorname{Id}_{\ell+1}+\operatorname{Rev}_{k+1}\right)$.

Teorema 3.7. Se $k \geqslant \ell \geqslant 2$ então temos $z^{\prime}\left(\operatorname{Id}_{\ell+1}+\operatorname{Rev}_{k+1}\right)=1 / \ell^{k}$. 
Este capítulo está organizado da seguinte forma: na Seção 3.1 provamos o Teorema 3.2 e na Seção 3.2 provamos o Teorema 3.7 .

\subsection{Problema de Empacotamento em Permutações}

Nessa seção, apresentamos as ferramentas necessárias para a prova do Teorema 3.2 assim como sua prova. Na Seção 3.1.1, apresentamos as adaptações feitas às ferramentas desenvolvidas por Price [21]. Na Seção 3.1.2, utilizamos as ferramentas desenvolvidas na Seção 3.1.1 para provar o Teorema 3.2.

\subsubsection{Adaptação do Algoritmo de Price}

O Algoritmo de Price, apresentado na Seção 2.1.2, gera cotas inferiores para o valor de empacotamento de uma permutação em camadas $\tau$ através de estimativas sobre a quantidade de camadas que uma permutação extremal de $\tau$ possui. Assim, apesar do Algoritmo de Price convergir para o valor de empacotamento de uma permutação em camada, ele se torna menos eficiente se a permutação extremal possuir uma anticamada, pois o algoritmo aproximará uma anticamada por uma sequência de camadas "pequenas". Com isso em mente, adaptamos o Algoritmo de Price de forma a considerar as anticamadas como uma estrutura única ao invés de tratá-las como várias camadas de tamanho 1. A intuição é construir polinômios análogos ao Polinômio de Price, mas alternando variáveis que representam anticamadas e camadas.

Definição 3.8. Seja $\tau \in \mathfrak{S}_{m}^{L}$ uma permutação em camadas de tamanho $m>0$. Definimos o Polinômio de Price Estendido de ordem $n \in \mathbb{N}$ para $\tau$ como o polinômio com $2 n$ variáveis reais dado por

$$
g_{n, \tau}\left(x_{1}, x_{2}, \ldots, x_{2 n}\right)=m ! \sum_{\left(\ell_{j}, \xi_{j}\right)_{j=1}^{k} \in \mathcal{Q}_{\tau}} \sum_{v \in \mathcal{K}(2 n, k)} \prod_{j=1}^{k} \frac{x_{v(j)}^{\ell_{j}}}{\ell_{j} !} \mathbb{1}_{\left\{v(j) \bmod 2=\xi_{j} \text { ou } \ell_{j}=1\right\}},
$$

onde $\mathbb{1}$ denota a função indicadora.

Note que a função indicadora força que as variáveis com índices ímpares correspondam às subanticamadas de $\tau$ e as variáveis com índices pares correspondam às camadas de $\tau$. Generalizamos o Polinômio de Price Estendido de ordem $n \in \mathbb{N}$ para o conjunto $\mathbb{R S}^{L}$ linearmente na primeira coordenada, i.e, dado $f=\sum_{i=1}^{k} a_{i} \tau_{i} \in \mathbb{R S}^{L}$ temos

$$
g_{n, f} \equiv \sum_{i=1}^{k} a_{i} g_{n, \tau_{i}}
$$

A Cota Estendida de Price, definida abaixo, possui outro parâmetro que é responsável por forçar que certas anticamadas de permutações "arbitrariamente grandes" possuam tamanho 0.

Definição 3.9 (Cota Estendida de Price). Sejam $f \in \mathbb{R S}^{L}$ uma combinação linear de permutações em camadas, $n \in \mathbb{N}$ um inteiro positivo e $W \subseteq[n]$ um subconjunto fixo. A Cota Estendida de Price de ordem $n$ relacionada a $W$ para $f$, denotada por $\mathcal{L}_{n, W, f}$, é definida como 


$$
\mathcal{L}_{n, W, f}=\max \left\{g_{n, f}\left(x_{1}, x_{2}, \ldots, x_{2 n}\right): \sum_{j=1}^{2 n} x_{j}=1 \text { e } \forall j \in[n], x_{j} \geqslant 0 \text { e } \forall j \in W, x_{2 j-1}=0\right\} .
$$

Note que, por argumentos de compacidade, o máximo acima existe e denotamos $\mathcal{L}_{n,[n], f}$ por $\mathcal{L}_{n, f}$. Vamos mostrar que essas cotas formam uma sequência que converge para o valor de empacotamento, i.e., vamos mostrar que a versão estendida do Algoritmo de Price funciona como desejado.

Teorema 3.10. Se $f=\sum_{t=1}^{k} a_{t} \tau_{t} \in \mathbb{R S}^{L}$ é uma combinação linear cônica de permutações em camadas e $\left(W_{n}\right)_{n \in \mathbb{N}}$ é uma sequência de conjuntos tais que $W_{n} \subseteq[n]$ para todo $n \in \mathbb{N}$ então

$$
\lim _{n \rightarrow \infty} \mathcal{L}_{n, W_{n}, f}=p(f) .
$$

Demonstração. Note que se $x_{1}, \ldots, x_{n} \geqslant 0$ são tais que $\sum_{j=1}^{n} x_{j}=1$ e $q_{n, f}\left(x_{1}, \ldots, x_{n}\right)=\mathcal{L}_{n, f}$ então

$$
\mathcal{L}_{n, W_{n}, f} \geqslant g_{n, f}\left(0, x_{1}, 0, x_{2}, \ldots, 0, x_{n}\right)=q_{n, f}\left(x_{1}, x_{2}, \ldots, x_{n}\right)=\mathcal{L}_{n, f} .
$$

Portanto, pela Proposição 2.8, temos $\liminf _{n \rightarrow \infty} \mathcal{L}_{n, W_{n}, f} \geqslant p(f)$. Agora, vamos mostrar a outra desigualdade com limsup. Dado $n \in \mathbb{N}$, sejam $\varepsilon>0$ um número real fixo e $b_{1}, \ldots, b_{2 n}, N \in \mathbb{N}$ com $N \neq 0$ tais que

- $\sum_{j=1}^{2 n} b_{j}=N$;

- $b_{2 j-1}=0$ para todo $j \in W_{n}$;

- $b_{2 j} \neq 0$ para todo $j \in[n]$;

- $g_{n, f}\left(\frac{b_{1}}{N}, \frac{b_{2}}{N}, \ldots, \frac{b_{2 n}}{N}\right) \geqslant \mathcal{L}_{n, W_{n}, f}-\varepsilon$.

Para todo $m \in \mathbb{N}$, seja $\sigma_{m} \in \mathfrak{S}_{m N}^{L}$ a permutação em camadas de tamanho $m N$ e com decomposição em blocos $\left(\widehat{m b_{1}}, m b_{2}, \widehat{m b_{3}}, m b_{4}, \ldots, \widehat{m b_{2 n-1}}, m b_{2 n}\right)$ (se qualquer um desses números for zero, removemos eles da decomposição que forma $\sigma_{m}$ ). Agora, para qualquer $t \in[k]$, contamos a quantidade de ocorrências de $\tau_{t}$ em $\sigma_{m}$ de acordo com a decomposição natural que eles induzem em $\tau_{t}$. Dessa forma, temos

$$
\begin{aligned}
p\left(\tau_{t}, \sigma_{m}\right) & =\left(\begin{array}{c}
m N \\
\left|\tau_{t}\right|
\end{array}\right)^{-1} \sum_{\left(\ell_{j}, \xi_{j}\right)_{j=1}^{k} \in \mathcal{Q}_{\tau_{t}}} \sum_{v \in \mathcal{K}(2 n, k)} \prod_{j=1}^{k}\left(\begin{array}{c}
m b_{v(j)} \\
\ell_{j}
\end{array}\right) \mathbb{1}_{\left\{v(j) \bmod 2=\xi_{j} \text { ou } \ell_{j}=1\right\}} \\
& \sim \frac{\left|\tau_{t}\right| !}{(m N)^{\left|\tau_{t}\right|}} \sum_{\left(\ell_{j}, \xi_{j}\right)_{j=1}^{k} \in \mathcal{Q}_{\tau_{t}}} \sum_{v \in \mathcal{K}(2 n, k)} \prod_{j=1}^{k} \frac{\left(m b_{v(j)}\right)^{\ell_{j}}}{\ell_{j} !} \mathbb{1}_{\left\{v(j) \bmod 2=\xi_{j} \text { ou } \ell_{j}=1\right\}} \\
& =\left|\tau_{t}\right| ! \sum_{\left(\ell_{j}, \xi_{j}\right)_{j=1}^{k} \in \mathcal{Q}_{\tau_{t}}} \sum_{v \in \mathcal{K}(2 n, k)} \prod_{j=1}^{k} \frac{\left(b_{v(j)} / N\right)^{\ell_{j}}}{\ell_{j} !} \mathbb{1}_{\left\{v(j) \bmod 2=\xi_{j} \text { ou } \ell_{j}=1\right\} .} . \\
& =g_{n, \tau_{t}}\left(\frac{b_{1}}{N}, \frac{b_{2}}{N}, \ldots, \frac{b_{2 n}}{N}\right),
\end{aligned}
$$


onde $\sim$ significa que a diferença entre ambos os lados tende a 1 quando $m$ tende a $\infty$ e a primeira igualdade após $\sim$ segue do fato de que $\sum_{j=1}^{k} \ell_{j}=\left|\tau_{t}\right|$ para toda decomposição em blocos $\left(\ell_{j}, \xi_{j}\right)_{j=1}^{k}$ de $\tau_{t}$. Portanto, temos

$$
\begin{aligned}
p\left(f, \sigma_{m}\right) & =\sum_{t=1}^{k} a_{t} p\left(\tau_{t}, \sigma_{m}\right) \\
& \sim \sum_{t=1}^{k} a_{t} g_{n, \tau_{t}}\left(\frac{b_{1}}{N}, \frac{b_{2}}{N}, \ldots, \frac{b_{2 n}}{N}\right) \\
& =g_{n, f}\left(\frac{b_{1}}{N}, \frac{b_{2}}{N}, \ldots, \frac{b_{2 n}}{N}\right) \\
& \geqslant \mathcal{L}_{n, W_{n}, f}-\varepsilon .
\end{aligned}
$$

Assim, para todo $\varepsilon>0$, temos

$$
p(f) \geqslant \lim _{m \rightarrow \infty} p\left(f, \sigma_{m}\right) \geqslant \mathcal{L}_{n, W_{n}, f}-\varepsilon .
$$

Como $\varepsilon$ é arbitrário, temos que $\mathcal{L}_{n, W_{n}, f} \leqslant p(f)$ para todo $n \in \mathbb{N}$, consequentemente

$$
p(f) \geqslant \limsup _{n \rightarrow \infty} \mathcal{L}_{n, W_{n}, f}
$$

De onde, concluímos que $\lim _{n \rightarrow \infty} \mathcal{L}_{n, W_{n}, f}=p(f)$.

\subsubsection{Resultado sobre o Problema de Empacotamento}

A prova do Teorema 3.2 é baseada nas provas apresentadas em [12, Teorema 3.3] e [27, Teorema 3.8]. Relembre que o Polinômio Estendido de Price pode ser visto como a função $p(\cdot)$ aplicada em $\tau$ e em uma permutação em camadas arbitrariamente grande, um número fixo de camadas (digamos $N$ ) e um número fixo de anticamadas. A demonstração do Teorema 3.2 é dividida em três passos. O primeiro passo (Lema 3.11) consiste em mostrar que, para a família de permutações em camadas descrita no enunciado do teorema, podemos adicionar uma restrição à Cota de Price Estendida afirmando que as variáveis relacionadas às camadas estão em ordem não decrescente. No segundo passo (Lema 3.12), mostramos que podemos ainda adicionar a restrição que o tamanho da primeira variável do polinômio não excede o tamanho da $(N-k+1)$-ésima variável. Por fim, no terceiro e último passo (Lema 3.13), mostramos que se $N>k$ então podemos juntar a segunda variável do polinômio, que agora é a menor variável relativa às camadas, com a primeira variável, que é relacionada à anticamada e assim reduzindo a quantidade de variáveis necessárias.

Dados inteiros positivos $2 \leqslant a \leqslant \ell_{1} \leqslant \cdots \leqslant \ell_{k}$, seja $\sigma$ uma permutação em camadas da forma $\left(\widehat{a}, \ell_{1}, \ldots, \ell_{k}\right)$. Sejam $\ell=a+\sum_{i=1}^{k} \ell_{i}$ o tamanho de $\sigma$ e tome $\ell_{i}=1$ para todo $i \leqslant 0$. Para 
todo $N \in \mathbb{N}^{*}$, defina

$$
\begin{aligned}
p_{N}\left(y, x_{1}, x_{2}, \ldots, x_{N}\right) & =\frac{\prod_{i=1}^{k} \ell_{i} !}{\ell !} g_{N, \sigma}\left(y, x_{1}, 0, x_{2}, 0, x_{3}, \ldots, 0, x_{N}\right) \\
& =\sum_{u=0}^{a} \frac{y^{u}}{u !} \sum_{v \in \mathcal{K}(N, a-u+k)}\left(\prod_{j=1}^{a-u+k} x_{v(j)}^{\ell_{j-a+u}}\right) \\
& =\sum_{u=0}^{a} \frac{y^{u}}{u !} \sum_{I \in\left(\begin{array}{c}
{[N]} \\
a-u+k
\end{array}\right)} x_{I}^{\ell^{[-a+u+1 . . k]}}
\end{aligned}
$$

onde $[-a+u+1 . . k]$ denota o conjunto $\{-a+u+1,-a+u+2, \ldots, k\}$.

Note que

$$
\begin{gathered}
\mathcal{L}_{N,[N] \backslash\{1\}, \sigma}=\max \left\{\frac{\ell !}{\prod_{i=1}^{k} \ell_{i} !} p_{N}\left(y, x_{1}, \ldots, x_{N}\right): y+\sum_{j=1}^{N} x_{j}=1\right. \\
\left.\operatorname{e~} \forall j \in[N], x_{j} \geqslant 0 \text { e } y \geqslant 0\right\} .
\end{gathered}
$$

Mais ainda, pelo Teorema 3.10 aplicado a $f=\sigma$ e $W_{N}=[N] \backslash\{1\}$ para todo $N \in \mathbb{N}$, temos

$$
p(\sigma)=\lim _{N \rightarrow \infty} \mathcal{L}_{N,[N] \backslash\{1\}, \sigma}
$$

Lema 3.11. Sejam $2 \leqslant a \leqslant \ell_{1} \leqslant \cdots \leqslant \ell_{k}$ inteiros e $\sigma$ uma permutação da forma $\left(\widehat{a}, \ell_{1}, \ldots, \ell_{k}\right)$, com $|\sigma|=\ell$. Para todo $N \geqslant k$ e $i \in[N-1]$, temos

$$
p_{N}\left(y, x_{1}, x_{2}, \ldots, x_{N}\right) \leqslant p_{N}\left(y, x_{1}, x_{2}, \ldots, x_{i-1}, x_{i+1}, x_{i}, x_{i+2}, \ldots, x_{N}\right),
$$

sempre que $y, x_{1}, x_{2}, \ldots, x_{N} \geqslant 0$ e $x_{i} \geqslant x_{i+1}$.

Demonstração. Para esta prova, denotamos por $p_{N}\left(y, x_{i} \leftrightarrow x_{i+1}\right)$ o valor

$$
p_{N}\left(y, x_{1}, x_{2}, \ldots, x_{i-1}, x_{i+1}, x_{i}, x_{i+2}, \ldots, x_{N}\right),
$$

onde trocamos as posições das variáveis $x_{i}$ e $x_{i+1}$ de $p_{N}$.

Estudamos agora a diferença $p_{N}\left(y, x_{i} \leftrightarrow x_{i+1}\right)-p_{N}\left(y, x_{1}, \ldots, x_{N}\right)$. Note que utilizando a forma (3.1) para calcular a diferença temos que os termos em $I$ não contêm $i$ ou $i+1$ se anulam. Mais ainda, os termos de $p_{N}\left(y, x_{i} \leftrightarrow x_{i+1}\right)$ no qual $I$ contém $i$ porém não contém $i+1$ se anula com o termo de $p_{N}\left(y, x_{1}, \ldots, x_{N}\right)$ em que $I$ contém $i+1$ mas não contém $i$, pois no primeiro, os valores $x_{i}$ e $x_{i+1}$ são trocados. Analogamente, os termos de $p_{N}\left(y, x_{i} \leftrightarrow x_{i+1}\right)$ nos quais $I$ contém $i+1$ mas não contém $i$ se anulam com os termos de $p_{N}\left(y, x_{1}, \ldots, x_{N}\right)$ em que $I$ contém $i$ 
mas não contém $i+1$. Dessa forma, temos

$$
\begin{aligned}
& p_{N}\left(y, x_{i} \leftrightarrow x_{i+1}\right)-p_{N}\left(y, x_{1}, x_{2}, \ldots, x_{N}\right) \\
= & \sum_{u=0}^{a} \frac{y^{u}}{u !} \sum_{\substack{j=1 \\
I_{\{j\}}=i, I_{\{j+1\}}=i+1}} \sum_{\substack{[N] \\
a-u+k}} x_{I \backslash\{i, i+1\}}^{\ell_{[-a+u+1 . k] \backslash\{j, j+1\}}}\left(x_{i+1}^{\ell_{j-a+u}} x_{i}^{\ell_{j+1-a+u}}-x_{i}^{\ell_{j-a+u}} x_{i+1}^{\ell_{j+1}-a+u}\right) .
\end{aligned}
$$

Agora, como $x_{i} \geqslant x_{i+1} \geqslant 0$ e

$$
\ell_{k} \geqslant \ell_{k-1} \geqslant \cdots \geqslant \ell_{1} \geqslant \ell_{0} \geqslant \ell_{-1} \geqslant \cdots \geqslant \ell_{1-a}
$$

temos que $x_{i+1}^{\ell_{t}} x_{i}^{\ell_{t+1}} \geqslant x_{i}^{\ell_{t}} x_{i+1}^{\ell_{t+1}}$ para todo $t<k$. Portanto

$$
p_{N}\left(y, x_{i} \leftrightarrow x_{i+1}\right)-p_{N}\left(y, x_{1}, x_{2}, \ldots, x_{N}\right) \geqslant 0
$$

O Lema 3.11 implica que podemos adicionar a restrição $x_{1} \leqslant x_{2} \leqslant \cdots \leqslant x_{N}$ para o máximo que define $\mathcal{L}_{N,[N] \backslash\{1\}, \sigma}$. O Lema 3.12 abaixo garante que podemos adicionar a restrição $y \leqslant x_{N-k+1}$ para o máximo que define $\mathcal{L}_{N,[N] \backslash\{1\}, \sigma}$

Lema 3.12. Sejam $2 \leqslant a \leqslant \ell_{1} \leqslant \cdots \leqslant \ell_{k}$ inteiros e $\sigma$ uma permutação da forma $\left(\widehat{a}, \ell_{1}, \ldots, \ell_{k}\right)$ com $|\sigma|=\ell$. Para todo $N>k$ temos

$$
\begin{gathered}
\mathcal{L}_{N,[N] \backslash\{1\}, \sigma}=\max \left\{\frac{\ell !}{\prod_{i=1}^{k} \ell_{i} !} p_{N}\left(y, x_{1}, x_{2}, \ldots, x_{N}\right): 0 \leqslant x_{1} \leqslant x_{2} \leqslant \cdots \leqslant x_{N},\right. \\
\left.0 \leqslant y \leqslant x_{N-k+1} \text { e } y+\sum_{j=1}^{N} x_{j}=1\right\} .
\end{gathered}
$$

Demonstração. A ideia geral da demonstração consiste no fato de que, se estamos em um ponto ótimo, então qualquer variação da função ao redor do ponto ótimo apenas piora o valor da função. Suponha que não possamos adicionar a restrição $y \leqslant x_{N-k+1}$ e sejam $y, x_{1}, x_{2}, \ldots, x_{N} \geqslant 0$ tais que

- Pelo Lema 3.11 podemos considerar $x_{1} \leqslant x_{2} \leqslant \cdots \leqslant x_{N}$;

- $y+\sum_{j=1}^{N} x_{j}=1$;

- $\mathcal{L}_{N,[N] \backslash\{1\}, \sigma}=\frac{\ell !}{\prod_{i=1}^{k} \ell_{i} !} p_{N}\left(y, x_{1}, x_{2}, \ldots, x_{N}\right)$;

- $y>x_{N-k+1}$.

Para todo $t \in \mathbb{R}$, seja

$$
f(t)=p_{N}\left(y-t, x_{1}, x_{2}, \ldots, x_{N-k}, x_{N-k+1}+t, x_{N-k+2}, \ldots, x_{N}\right) .
$$

Assim, temos

$$
\mathcal{L}_{N,[N] \backslash\{1\}, \sigma} \geqslant \frac{\ell !}{\prod_{i=1}^{k} \ell_{i} !} f(t)
$$

com igualdade se $t=0$ (podemos perder a condição $x_{1} \leqslant x_{2} \leqslant \cdots \leqslant x_{N}$ para $t>x_{N-k+2}-x_{N-k+1}$ ). 
Como $f$ é diferenciável, temos que

$$
f^{\prime}(0) \leqslant 0 .
$$

Note que

$$
f^{\prime}(0)=-\sum_{u=0}^{a} \frac{u y^{u-1}}{u !} \sum_{\substack{[N] \\
I \in-u+k}} x_{I-a+u+1 . . k]}^{\ell^{[-a}}+\sum_{u=0}^{a} \frac{y^{u}}{u !} \sum_{j=a-u+1}^{a-u+k} \sum_{\substack{I \in\left(\begin{array}{c}
{[N] \\
a-u+k}
\end{array}\right) \\
I_{\{j\}}=N-k+1}} x_{I-a+u+1 . . k] \backslash\{j\}}^{\ell_{[-k+1\}}} \ell_{j-a+u} x_{N-k+1}^{\ell_{j-a+u}-1},
$$

onde a primeira parte da soma segue do termo que contém $y-t$ e a segunda parte segue do termo que contém $x_{N-k+1}+t$.

Agora dividimos o primeiro somatório de acordo com quais $I$ possui o elemento $N-k+1$ e os que não possuem. Assim,

$$
\begin{aligned}
& \sum_{u=0}^{a} \frac{u y^{u-1}}{u !} \sum_{I \in\left(\begin{array}{c}
{[N]} \\
a-u+k
\end{array}\right)} x_{I}^{\ell_{[-a+u+1 . . k]}}
\end{aligned}
$$

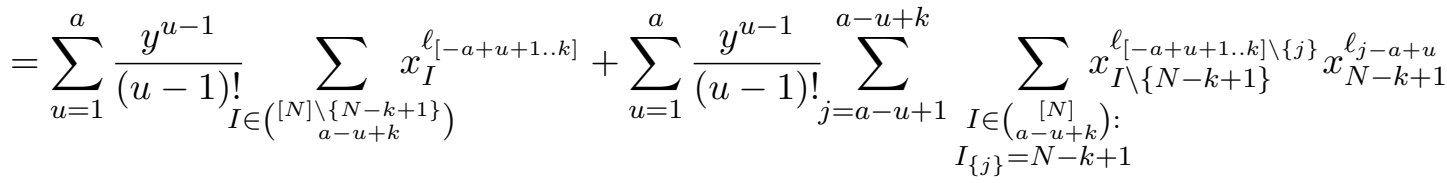

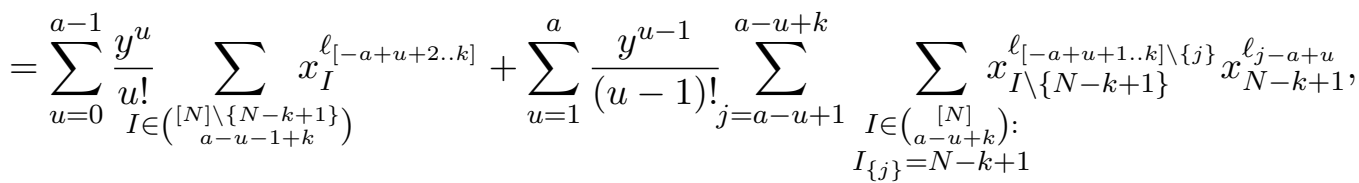

onde na última igualdade aplicamos a mudança de variável $u \rightarrow u+1$ no primeiro somatório. Aplicando (3.3) em (3.2), temos

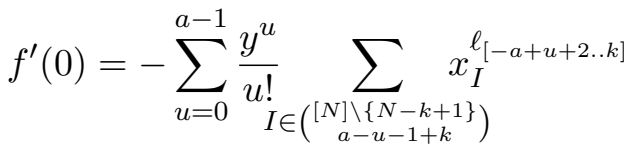

$$
\begin{aligned}
& +\sum_{u=1}^{a} \frac{y^{u-1}}{(u-1) !} \sum_{j=a-u+1}^{a-u+k} \sum_{\substack{I \in\left(\begin{array}{c}
{[N] \\
a-u+k}
\end{array}\right): \\
I_{\{j\}}=N-k+1}} x_{I \backslash\{N-k+1\}}^{\ell_{[-a+u+1 . k] \backslash\{j\}}} x_{N-k+1}^{\ell_{j-a+u}-1}\left(\frac{y}{u} \ell_{j-a+u}-x_{N-k+1}\right)
\end{aligned}
$$

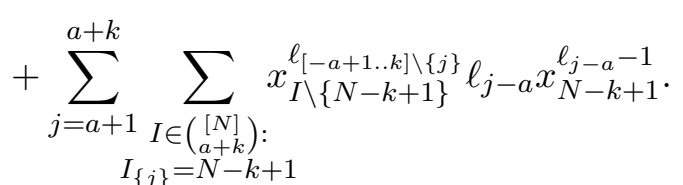

Agora vamos obter uma cota inferior para os dois últimos somatórios. Primeiro, note que, para todo $u \in[a]$ e todo $a-u+1 \leqslant j \leqslant a-u+k$, temos

$$
\frac{y}{u} \ell_{j-a+u}-x_{N-k+1} \geqslant \frac{y}{u} \ell_{1}-x_{N-k+1} \geqslant 0,
$$


pois $u \leqslant a \leqslant \ell_{1}$ e $y>x_{N-k+1}$. Utilizando (3.5) e o Lema (2.1), temos

$$
\begin{aligned}
& \sum_{j=a-u+1}^{a-u+k} \sum_{\substack{I \in\left(\begin{array}{c}
{[N] \\
a-u+k}
\end{array}\right): \\
I_{\{j\}}=N-k+1}} x_{I \backslash\{N-k+1\}}^{\ell_{[-a+u+1 . k] \backslash\{j\}}} x_{N-k+1}^{\ell_{j-a+u}-1}\left(\frac{y}{u} \ell_{j-a+u}-x_{N-k+1}\right)
\end{aligned}
$$

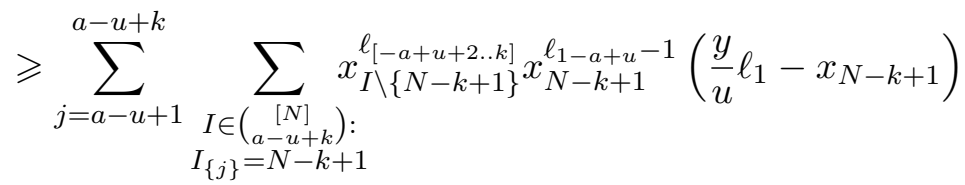

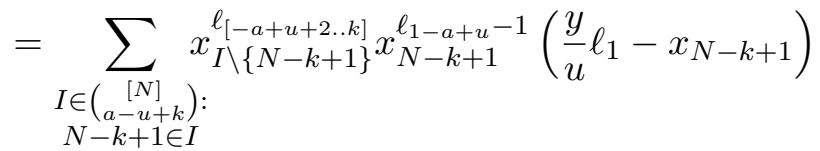

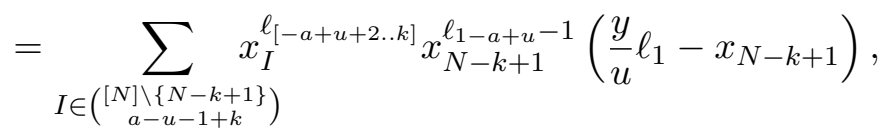

onde na última igualdade aplicamos a mudança de variável $I \rightarrow I \backslash\{N-k+1\}$. Analogamente, como $\ell_{1-a}=1$, temos

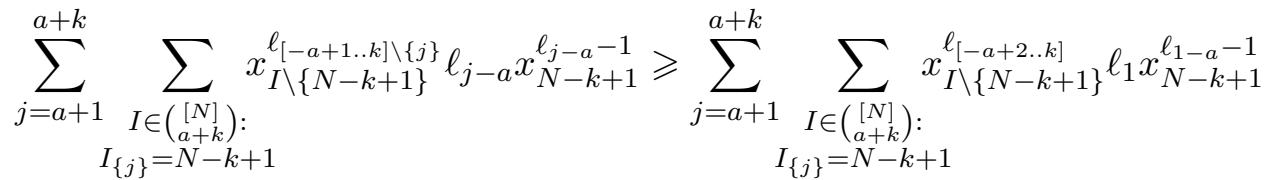

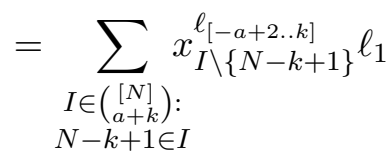

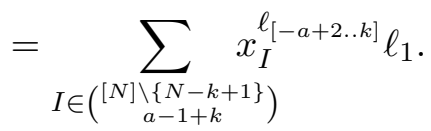

Combinando as desigualdades (3.6) e (3.7) em (3.4), temos

$$
\begin{aligned}
& f^{\prime}(0) \geqslant-\sum_{u=0}^{a-1} \frac{y^{u}}{u !} \sum_{I \in\left(\begin{array}{c}
{[N] \backslash\{N-k+1\}} \\
a-u-1+k
\end{array}\right)} x_{I}^{\ell_{[-a+u+2 . . k]}} \\
& +\sum_{u=1}^{a} \frac{y^{u-1}}{(u-1) !} \sum_{I \in\left(\begin{array}{c}
{[N] \backslash\{N-k+1\}} \\
a-u-1+k
\end{array}\right)} x_{I}^{\ell_{[-a+u+2 . . k]}} x_{N-k+1}^{\ell_{1-a+u}-1}\left(\frac{y}{u} \ell_{1}-x_{N-k+1}\right) \\
& +\sum_{I \in\left(\begin{array}{c}
{[N] \backslash\{N-k+1\}} \\
a-1+k
\end{array}\right)} x_{I}^{\ell_{[-a+2 . k]}} \ell_{1} \\
& =\sum_{u=1}^{a-1} \frac{y^{u-1}}{(u-1) !} \sum_{I \in\left(\begin{array}{c}
{[N] \backslash\{N-k+1\}} \\
a-u-1+k
\end{array}\right)}\left(\frac{y}{u} \ell_{1}-x_{N-k+1}-\frac{y}{u}\right) x_{I}^{\ell_{I-a+u+2 . . k]}} \\
& +\frac{y^{a-1}}{(a-1) !} \sum_{I \in\left(\begin{array}{c}
{[N] \backslash\{N-k+1\}} \\
k-1
\end{array}\right)}\left(\frac{y}{a} \ell_{1}-x_{N-k+1}\right) x_{I}^{\ell_{[2 . . k]}} x_{N-k+1}^{\ell_{1}-1} \\
& +\sum_{I \in\left(\begin{array}{c}
{[N] \backslash\{N-k+1\}} \\
a-1+k
\end{array}\right)} x_{I}^{\ell^{[-a+2 . . k]}}\left(\ell_{1}-1\right) \text {. }
\end{aligned}
$$


Note que, como $\ell_{1} \geqslant a$, para todo $u \in[a-1]$, temos

$$
\frac{y}{u} \ell_{1}-x_{N-k+1}-\frac{y}{u}=\frac{y}{u}\left(\ell_{1}-1\right)-x_{N-k+1} \geqslant \frac{\ell_{1}-1}{a-1} y-x_{N-k+1} \geqslant y-x_{N-k+1}>0 .
$$

Mais ainda, note que

$$
\frac{y}{a} \ell_{1}-x_{N-k+1} \geqslant y-x_{N-k+1}>0
$$

e também $\ell_{1}-1>0$, portanto temos $f^{\prime}(0)>0$, o que contradiz (3.2).

Provaremos agora que é suficiente considerar a Cota Estendida de Price de ordem $k$ relacionada a $[k] \backslash\{1\}$. Para isso, vamos utilizar o Lema 3.12 para adicionar a restrição de ordenação nas variáveis que definem a Cota Estendida de Price e mostraremos que o valor da cota não piora se combinarmos a primeira variável relativa às camadas com a variável $y$.

Lema 3.13. Sejam $2 \leqslant a \leqslant \ell_{1} \leqslant \cdots \leqslant \ell_{k}$ inteiros, com $2^{a}-a-1 \geqslant k$ e $\sigma$ uma permutação da forma $\left(\widehat{a}, \ell_{1}, \ldots, \ell_{k}\right) \operatorname{com}|\sigma|=\ell$. Para todo $N>k$, temos

$$
\mathcal{L}_{N,[N] \backslash\{1\}, \sigma} \leqslant \mathcal{L}_{N-1,[N-1] \backslash\{1\}, \sigma} .
$$

Demonstração. Pelos Lemas 3.11 e 3.12 , sabemos que existem $y, x_{1}, x_{2}, \ldots, x_{N} \geqslant 0$ tais que

- $x_{1} \leqslant x_{2} \leqslant \cdots \leqslant x_{N}$

- $y+\sum_{j=1}^{N} x_{j}=1$

- $y \leqslant x_{N-k+1}$;

- $\mathcal{L}_{N,[N] \backslash\{1\}, \sigma}=\frac{\ell !}{\prod_{i=1}^{k} \ell_{i} !} p_{N}\left(y, x_{1}, x_{2}, \ldots, x_{N}\right)$.

Consideramos agora o que acontece quando usamos a Extensão do Polinômio de Price de ordem $N-1$ no ponto $\left(y+x_{1}, x_{2}, x_{3}, \ldots, x_{N}\right)$. Isso corresponde a combinar os dois primeiros blocos de $p_{N}$ em uma anticamada. Note que

$$
\mathcal{L}_{N-1,[N-1] \backslash\{1\}, \sigma} \geqslant \frac{\ell !}{\prod_{i=1}^{k} \ell_{i} !} p_{N-1}\left(y+x_{1}, x_{2}, x_{3}, \ldots, x_{N}\right) .
$$

Portanto, é suficiente mostrar que $p_{N-1}\left(y+x_{1}, x_{2}, x_{3}, \ldots, x_{N}\right) \geqslant p_{N}\left(y, x_{1}, x_{2}, \ldots, x_{N}\right)$. Para isso, reorganizaremos o somatório que ocorre em $p_{N-1}$ e em $p_{N}$ de tal forma que, quando calcularmos a diferença entre eles, os termos comuns em entre eles se anulem.

Primeiro, dividimos o somatório da definição de $p_{N}$ de acordo com os termos que possuem $x_{1}$ 
e os que não possuem. Dessa forma, temos que

$$
\begin{aligned}
& p_{N}\left(y, x_{1}, x_{2}, \ldots, x_{N}\right) \\
& =\sum_{u=0}^{a} \frac{y^{u}}{u !} \sum_{I \in\left(\begin{array}{c}
{[N]} \\
a-u+k
\end{array}\right)} x_{I}^{\ell[-a+u+1 . . k]}
\end{aligned}
$$

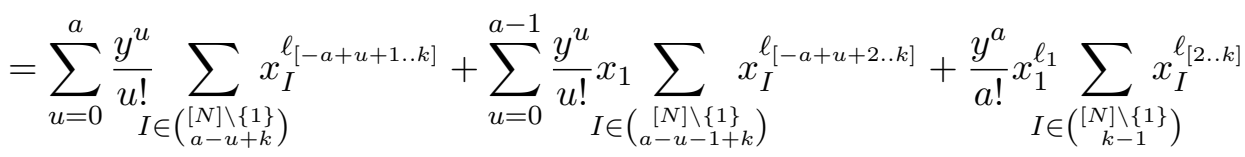

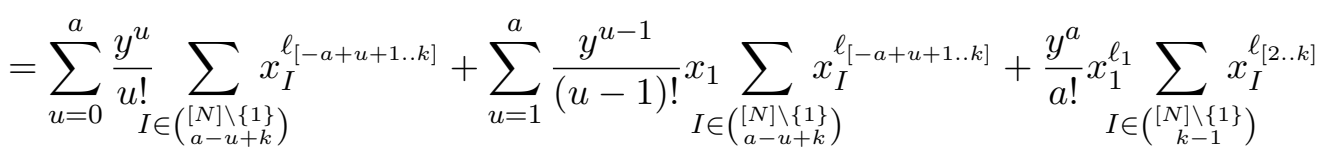

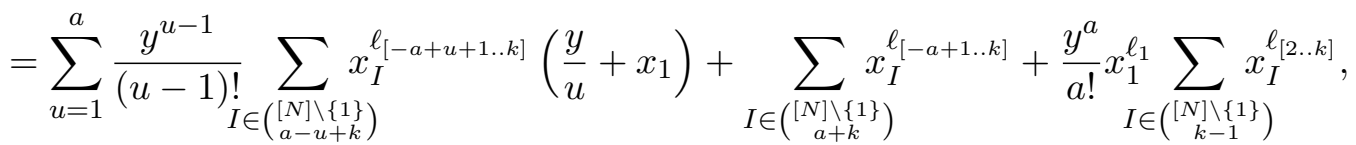

onde na terceira igualdade aplicamos a mudança de variável $u$ para $u-1$ no segundo somatório. Por outro lado, podemos separar os termos de $p_{N-1}$ que possuem $u=0$, obtendo

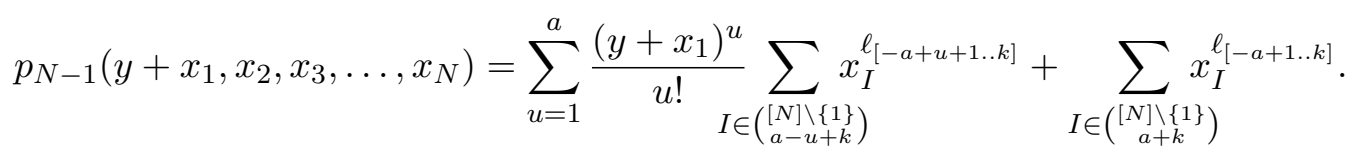

Dessa forma, calculando a diferença, temos

$$
\begin{aligned}
& p_{N-1}\left(y+x_{1}, x_{2}, x_{3}, \ldots, x_{N}\right)-p_{N}\left(y, x_{1}, x_{2}, \ldots, x_{N}\right)
\end{aligned}
$$

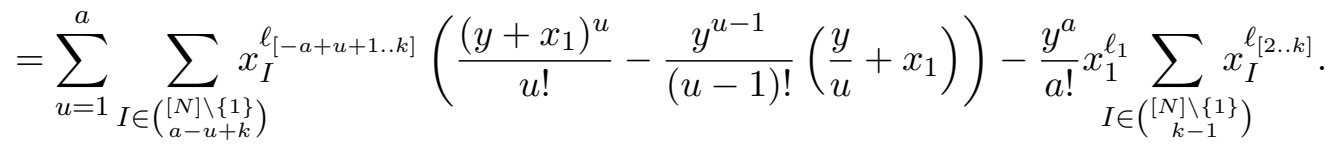

Agora, para todo $u \in[a]$, temos

$$
\left(\frac{\left(y+x_{1}\right)^{u}}{u !}-\frac{y^{u-1}}{(u-1) !}\left(\frac{y}{u}+x_{1}\right)\right)=\frac{1}{u !}\left(\left(y+x_{1}\right)^{u}-y^{u}-u x_{1} y^{u-1}\right)=\frac{1}{u !} \sum_{v=0}^{u-2}\left(\begin{array}{l}
u \\
v
\end{array}\right) y^{v} x_{1}^{u-v} \geqslant 0 .
$$

Isso garante que

$$
\begin{aligned}
& p_{N-1}\left(y+x_{1}, x_{2}, x_{3}, \ldots, x_{N}\right)-p_{N}\left(y, x_{1}, x_{2}, \ldots, x_{N}\right) \\
\geqslant & \frac{1}{a !} \sum_{v=0}^{a-2}\left(\begin{array}{l}
a \\
v
\end{array}\right) y^{v} x_{1}^{a-v} \sum_{I \in\left(\begin{array}{c}
{[N] \backslash\{1\}} \\
k
\end{array}\right)} x_{I}^{\ell_{k}}-\frac{y^{a}}{a !} x_{1}^{\ell_{1}} \sum_{\substack{I \in([N] \backslash\{1\} \\
k-1}} x_{I}^{\ell_{[2 . k]}} .
\end{aligned}
$$


Note que

$$
\begin{aligned}
& \sum_{I \in\left(\begin{array}{c}
{[N] \backslash\{1\}} \\
k
\end{array}\right)} x_{I}^{\ell_{[k]}}=\sum_{I \in\left(\begin{array}{c}
{[N] \backslash\{1\}} \\
k
\end{array}\right)} \frac{1}{k} \sum_{i \in I} x_{I}^{\ell_{[k]}} \\
& \geqslant \sum_{I \in\left(\begin{array}{c}
{[N] \backslash\{1\}} \\
k
\end{array}\right)} \frac{1}{k} \sum_{i \in I} x_{I \backslash\{i\}}^{\ell_{[2 . k]}} x_{i}^{\ell_{1}} \\
& =\frac{1}{k} \sum_{i \in[N] \backslash\{1\}} x_{i}^{\ell_{1}} \sum_{\substack{I \in([N] \backslash\{1\} \\
k \\
i \in I}} x_{I \backslash\{i\}}^{\ell_{[2 . . k]}},
\end{aligned}
$$

onde a desigualdade segue do Lema 2.1.

Agora podemos aplicar a mudança de variável $I \rightarrow I \backslash\{i\}$ e obtemos

$$
\begin{aligned}
& \sum_{I \in\left(\begin{array}{c}
{[N] \backslash\{1\}} \\
k
\end{array}\right)} x_{I}^{\ell_{[k]}} \geqslant \frac{1}{k} \sum_{i \in[N] \backslash\{1\}} x_{i}^{\ell_{1}} \sum_{\substack{I \in([N] \backslash\{1\} \\
k-1 \\
i \notin I}} x_{I}^{\ell_{[2 . . k]}}
\end{aligned}
$$

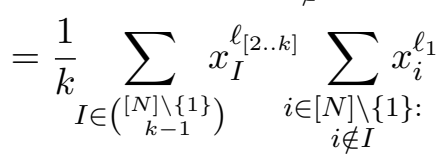

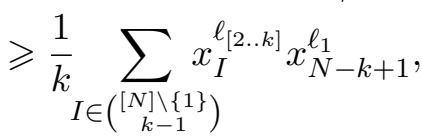

onde a última desigualdade segue do fato de que o somatório contém pelo menos um dos ter$\operatorname{mos} x_{N-k+1}^{\ell_{1}}, x_{N-k+2}^{\ell_{1}}, \ldots, x_{N}^{\ell_{1}}$ e eles são todos maiores ou iguais a $x_{N-k+1}^{\ell_{1}}$. Usando (3.8), temos

$$
\begin{aligned}
& p_{N-1}\left(y+x_{1}, x_{2}, x_{3}, \ldots, x_{N}\right)-p_{N}\left(y, x_{1}, x_{2}, \ldots, x_{N}\right) \\
\geqslant & \frac{1}{a !}\left(\frac{1}{k} \sum_{v=0}^{a-2}\left(\begin{array}{l}
a \\
v
\end{array}\right) y^{v} x_{1}^{a-v} x_{N-k+1}^{\ell_{1}}-y^{a} x_{1}^{\ell_{1}}\right) \sum_{I \in\left(\begin{array}{c}
{[N] \backslash\{1\}} \\
k-1
\end{array}\right)} x_{I}^{\ell_{[2 . . k]}} .
\end{aligned}
$$

Finalmente, como $x_{N-k+1} \geqslant x_{1}$ e $x_{N-k+1} \geqslant y$, temos $x_{N-k+1}^{\ell_{1}} \geqslant y^{a-u} x_{1}^{\ell_{1}-a+u}$. Dessa forma, obtemos

$$
\begin{aligned}
\frac{1}{k} \sum_{u=0}^{a-2}\left(\begin{array}{l}
a \\
u
\end{array}\right) y^{u} x_{1}^{a-u} x_{N-k+1}^{\ell_{1}}-y^{a} x_{1}^{\ell_{1}} & \geqslant \frac{1}{k} \sum_{u=0}^{a-2}\left(\begin{array}{l}
a \\
u
\end{array}\right) y^{a} x_{1}^{\ell_{1}}-y^{a} x_{1}^{\ell_{1}} \\
& =\frac{2^{a}-a-1}{k} y^{a} x_{1}^{\ell_{1}}-y^{a} x_{1}^{\ell_{1}} \\
& \geqslant 0,
\end{aligned}
$$

onde a última desigualdade segue da hipótese de que $2^{a}-a-1 \geqslant k$. Portanto, temos

$$
p_{N-1}\left(y+x_{1}, x_{2}, x_{3}, \ldots, x_{N}\right) \geqslant p_{N}\left(y, x_{1}, x_{2}, \ldots, x_{N}\right) .
$$

Note que o Teorema 3.2 é consequência imediata do Lema 3.13.

Prova do Teorema 3.2. Dados inteiros positivos $2 \leqslant a \leqslant \ell_{1} \leqslant \cdots \leqslant \ell_{k}$, com $2^{a}-a-1 \geqslant k$, seja $\sigma$ uma permutação em camadas da forma $\left(\widehat{a}, \ell_{1}, \ldots, \ell_{k}\right)$. Sejam $\ell=a+\sum_{i=1}^{k} \ell_{i}$ o tamanho de $\sigma$. 
Pelo Teorema 3.10 aplicado a $f=\sigma$ e $W_{N}=[N] \backslash\{1\}$ para todo $N \in \mathbb{N}$, temos

$$
p(\sigma)=\lim _{N \rightarrow \infty} \mathcal{L}_{N,[N] \backslash\{1\}, \sigma} .
$$

Pelo Lema 3.13, temos que

$$
p(\sigma)=\lim _{N \rightarrow \infty} \mathcal{L}_{N,[N] \backslash\{1\}, \sigma}=\mathcal{L}_{k,[k] \backslash\{1\}, \sigma} .
$$

Utilizaremos os Multiplicadores de Lagrange (ver Teorema 2.4) para poder calcular o valor da função $p(\sigma)$. Para isso, vamos calcular o valor de $\mathcal{L}_{k,[k] \backslash\{1\}, \sigma}$.

$$
F\left(y, x_{1}, \ldots, x_{k}\right)=|\sigma| ! \frac{y^{a}}{a !} \prod_{i=1}^{k} \frac{x_{i}^{\ell_{i}}}{\ell_{i} !}, \quad G\left(y, x_{1}, \ldots, x_{k}\right)=\left(y+\sum_{i=1}^{k} x_{i}\right)-1,
$$

$U=(0,1)^{k+1}$ e $D=\left\{\left(y, x_{1}, \ldots, x_{k}\right) \in U: G\left(y, x_{1}, \ldots, x_{k}\right)=0\right\}$. Note que estamos assumindo que $y>0$ e $x_{i}>0$ para todo $i \in[k]$, pois sabemos que $\mathcal{L}_{k,[k] \backslash\{1\}, \sigma}>0$. Como $\nabla G=(1, \ldots, 1)$, para todo $1 \leqslant i \leqslant k$ temos

$$
\frac{\partial F\left(y, x_{1}, \ldots, x_{k}\right)}{\partial x_{i}}=F\left(y, x_{1}, \ldots, x_{k}\right) \frac{\ell_{i}}{x_{i}} \quad \text { e } \quad \frac{\partial F\left(y, x_{1}, \ldots, x_{k}\right)}{\partial y}=F\left(y, x_{1}, \ldots, x_{k}\right) \frac{a}{y},
$$

utilizando os Multiplicadores de Lagrange (ver Teorema 2.4), temos que se $w \in D$ é um ponto de máximo local de $F$ então $\nabla F(w)$ é múltiplo de $(1, \ldots, 1)$. Portanto, para todo $1 \leqslant i \leqslant k$, temos

$$
F\left(y, x_{1}, \ldots, x_{k}\right) \frac{\ell_{i}}{x_{i}}=F\left(y, x_{1}, \ldots, x_{k}\right) \frac{a}{y}=F\left(y, x_{1}, \ldots, x_{k}\right) \frac{\ell_{1}}{x_{1}} .
$$

Como $G\left(x_{1}, \ldots, x_{k}\right)=0$, temos $x_{i}=\ell_{i} /|\sigma|$ para todo $1 \leqslant i \leqslant k$ e $y=a /|\sigma|$. Assim, concluímos que

$$
p(\sigma)=\frac{|\sigma| !}{|\sigma| \sigma \mid} \frac{a^{a}}{a !} \prod_{i=1}^{k} \frac{\ell_{i}^{\ell_{i}}}{\ell_{i} !}
$$

O Corolário 3.14 abaixo segue de um argumento apresentado em [12, Lema 3.4]. De forma mais simples, o corolário abaixo diz que o Teorema 3.2 permanece válido mesmo se mudarmos a ordem dos blocos.

Corolário 3.14. Sejam $a, k \in \mathbb{N}^{*}$ inteiros positivos tais que $a \geqslant 2$ e $2^{a}-a-1 \geqslant k$. Seja $\sigma$ uma permutação em camadas contendo exatamente uma anticamada de tamanho $a$ e $k$ camadas de tamanho pelo menos 2. Suponha que o tamanho de todas essas $k$ camadas são maiores ou iguais a $a$. Se a anticamada é o $j$-ésimo bloco de $\sigma$ e $j<k$ então temos

$$
p(\sigma)=\mathcal{L}_{k,[k] \backslash\{j\}, \sigma} .
$$

Demonstração. Sejam $2 \leqslant a \leqslant \ell_{1} \leqslant \ell_{2} \leqslant \cdots \leqslant \ell_{k}$ os tamanhos das camadas de $\sigma$ e $\sigma^{\prime}=$ $\left(\widehat{a}, \ell_{1}, \ell_{2}, \ldots, \ell_{k}\right)$ uma permutação em camadas. Primeiro provamos que para todo $N \in \mathbb{N}^{*}$, te- 
$\operatorname{mos} \mathcal{L}_{N, \sigma} \leqslant \mathcal{L}_{N, \sigma^{\prime}}$ (note que estamos usando a Cota de Price).

Seja $\left(r_{1}, r_{2}, \ldots, r_{m}\right)$ a sequência de camadas de $\sigma$ (note que a sequência inclui os $\ell_{i}$ 's em alguma ordem e então inclui a sequência de 1's de tamanho $a$, portanto $m=k+a)$. Fixe $x=\left(x_{1}, x_{2}, \ldots, x_{N}\right)$ tal que temos $\sum_{i=1}^{N} x_{i}=1$, para todo $i \in[N]$ temos $x_{i} \geqslant 0$ e

$$
q_{N, \sigma}(x)=\mathcal{L}_{N, \sigma}
$$

Para toda sequência de números reais $s$, seja $s \leqslant$ a sequência ordenada de $s$. Note que

$$
\begin{aligned}
q_{N, \sigma}(x) & =\frac{|\sigma| !}{\prod_{i=1}^{m} r_{i} !} \sum_{I \in\left(\begin{array}{c}
{[N]} \\
m
\end{array}\right)} x_{I[m]}^{r_{[m}} \leqslant \frac{|\sigma| !}{\prod_{i=1}^{m} r_{i} !} \sum_{I \in\left(\begin{array}{c}
{[N]} \\
m
\end{array}\right)}\left(x_{I}\right)^{\left(r_{[m]}\right)} \leqslant \\
& =\frac{|\sigma| !}{\prod_{i=1}^{m} r_{i} !} \sum_{I \in\left(\begin{array}{c}
{[N]} \\
m
\end{array}\right)}\left(x_{\leqslant}\right)_{I}^{\left(r_{[m]}\right)} \leqslant=q_{N, \sigma^{\prime}}\left(x_{\leqslant}\right) \leqslant \mathcal{L}_{N, \sigma^{\prime}} .
\end{aligned}
$$

Portanto, temos $p(\sigma) \leqslant p\left(\sigma^{\prime}\right)$. Agora, pelo Teorema 3.2, sabemos que $p\left(\sigma^{\prime}\right)=\mathcal{L}_{k,[k] \backslash\{1\}, \sigma^{\prime}}$. Dessa forma, sejam $y, x_{1}, \ldots, x_{k} \geqslant 0$ tais que $y+\sum_{i=1}^{k} x_{i}=1 \mathrm{e}$

$$
g_{k, \sigma^{\prime}}\left(y, x_{1}, 0, x_{2}, 0, x_{3}, \ldots, 0, x_{k}\right)=\mathcal{L}_{k,[k] \backslash\{1\}, \sigma^{\prime}}
$$

Seja $\tau$ uma permutação tal que a sequência de blocos de $\sigma$ é

$$
\left(\ell_{\tau(1)}, \ell_{\tau(2)}, \ldots, \ell_{\tau(j-1)}, \widehat{a}, \ell_{\tau(j)}, \ldots, \ell_{\tau(k)}\right)
$$

Note que

$$
\begin{aligned}
p\left(\sigma^{\prime}\right) & =\mathcal{L}_{k,[k] \backslash\{1\}, \sigma^{\prime}}=g_{k, \sigma^{\prime}}\left(y, x_{1}, 0, x_{2}, 0, x_{3}, \ldots, 0, x_{k}\right) \\
& =\frac{\left|\sigma^{\prime}\right| !}{a ! \prod_{i=1}^{k} \ell_{i} !} y^{a} x^{\ell[k]}=\frac{|\sigma| !}{a ! \prod_{i=1}^{k} \ell_{i} !} y^{a} \prod_{i=1}^{k} x_{\tau(i)}^{\ell_{\tau(i)}} \\
& =g_{k, \sigma}\left(0, x_{\tau(1)}, 0, x_{\tau(2)}, \ldots, 0, x_{\tau(j-1)}, y, x_{\tau(j)}, 0, x_{\tau(j+1)}, \ldots, 0, x_{\tau(k)}\right) \\
& \leqslant \mathcal{L}_{k,[k] \backslash\{j\}, \sigma} \leqslant p(\sigma) .
\end{aligned}
$$

Portanto, $p(\sigma)=p\left(\sigma^{\prime}\right)=\mathcal{L}_{k,[k] \backslash\{j\}, \sigma}$.

Observação 3.15. Note que não permitimos que uma anticamada seja o último bloco simplesmente pelo fato de que a Extensão do Polinômio de Price não termina com uma anticamada. Se um anticamada aparecer no último bloco de $\sigma$, não podemos aplicar o Corolário 3.14 diretamente. Porém, é fácil ver que $\sigma$ deve possuir a mesma densidade de empacotamento que a permutação que possui a mesma sequência de blocos de $\sigma$ de forma invertida e esta permutação não possui uma anticamada como último bloco.

\subsection{Problema de Minimização de Subsequências Monótonas}

Nesta seção, estudamos o problema dual do problema de empacotamento, ou seja, minimizar a densidade de permutações assintoticamente. Para isso, readaptamos algumas definições para o dual do problema de empacotamento. 
Definition 3.2.1. A Cota de Price de Minimização de ordem $n \in \mathbb{N}^{*}$ da combinação linear de permutações em camadas $f$, denotada por $\mathcal{U}_{n, f}$, é definida como

$$
\mathcal{U}_{n, f}=\min \left\{q_{n, f}\left(x_{1}, x_{2}, \ldots, x_{n}\right): \sum_{j=1}^{n} x_{j}=1 \text { e } \forall j \in[n], x_{j} \geqslant 0\right\} .
$$

Note que, por argumento de compacidade, esse mínimo existe e, portanto, a função é bem definida.

Para a Cota de Price de Minimização, obtemos os seguintes resultados que são análogos aos anteriores.

Teorema 3.16. Se $f \in \mathbb{R S}^{L}$ é uma combinação cônica de permutações em camadas então, para todo $n \in \mathbb{N}^{*}$, temos $\mathcal{U}_{n, f} \geqslant \mathcal{U}_{n+1, f}$ e

$$
\lim _{n \rightarrow \infty} \mathcal{U}_{n, f} \geqslant z^{\prime}(f)
$$

Em particular, temos $\lim _{n \rightarrow \infty} \mathcal{U}_{n, f}=z^{\prime}(f)$.

Demonstração. Análoga à prova do Teorema 3.10.

A seguir, apresentamos a prova do Teorema 3.7. A ideia da prova é a mesma do Teorema 3.2, i.e., a estratégia consiste em mostrar que não necessitamos de mais do que $\ell$ camadas para encontrar a permutação que realiza o mínimo. Em outras palavras, vamos mostrar que $\mathcal{U}_{N, \mathrm{Id}_{\ell}+\operatorname{Rev}_{k}} \geqslant$ $\mathcal{U}_{N-1, \mathrm{Id}_{\ell}+\operatorname{Rev}_{k}}$ sempre que $N \geqslant \ell$.

Prova do Teorema 3.7. Primeiro note que, para todo $N \in \mathbb{N}^{*}$, temos

$$
q_{N, \mathrm{Id}_{l}}+\operatorname{Rev}_{k}\left(x_{1}, x_{2}, \ldots, x_{N}\right)=\ell ! \sum_{I \in\left(\begin{array}{c}
{[N]} \\
\ell
\end{array}\right)} \prod_{i \in I} x_{i}+\sum_{i \in[N]} x_{i}^{k}
$$

Sejam $x_{1}, x_{2}, \ldots, x_{N} \geqslant 0$ com $\sum_{i=1}^{N} x_{i}=1$ tais que $q_{N, \mathrm{Id}_{\ell}}+\operatorname{Rev}_{k}\left(x_{1}, x_{2}, \ldots, x_{N}\right)=\mathcal{U}_{N, \mathrm{Id}_{\ell}}+\operatorname{Rev}_{k}$ e, sem perda de generalidade, pela simetria de $q_{N, \mathrm{Id}_{\ell}+\operatorname{Rev}_{k}}$ podemos supor que $x_{1} \leqslant x_{2} \leqslant \cdots \leqslant$

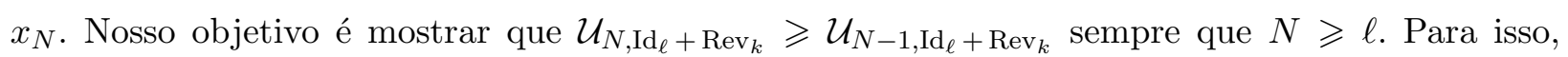
agrupamos os termos em $q_{N, \mathrm{Id}_{\ell}+\operatorname{Rev}_{k}}$ e $q_{N-1, \mathrm{Id}_{\ell}+\operatorname{Rev}_{k}}$ de acordo com os que possuem somente $x_{1}$, os que possuem somente $x_{2}$ ou os que possuem ambos. Dessa forma, note que

$$
\begin{aligned}
& q_{N, \operatorname{Id}_{\ell}}+\operatorname{Rev}_{k}\left(x_{1}, x_{2}, \ldots, x_{N}\right)
\end{aligned}
$$

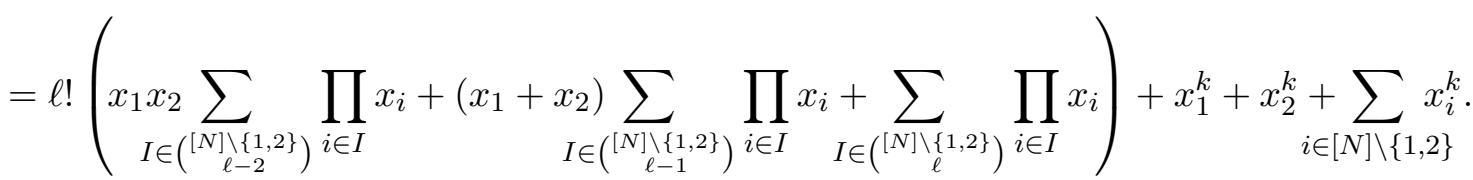

Por outro lado, temos

$$
\begin{aligned}
& q_{N-1, \operatorname{Id}_{\ell}}+\operatorname{Rev}_{k}\left(x_{1}+x_{2}, x_{3}, x_{4}, \ldots, x_{N}\right)
\end{aligned}
$$

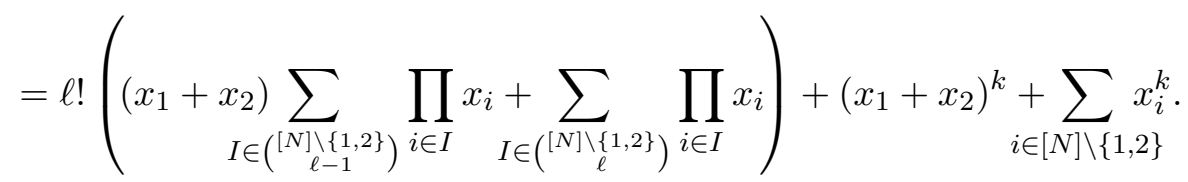


Subtraindo os polinômios (3.10) e (3.11), temos

$$
\begin{aligned}
& q_{N, \mathrm{Id}_{\ell}}+\operatorname{Rev}_{k}\left(x_{1}, x_{2}, \ldots, x_{N}\right)-q_{N-1, \mathrm{Id}_{\ell}+\operatorname{Rev}_{k}}\left(x_{1}+x_{2}, x_{3}, x_{4}, \ldots, x_{N}\right) \\
= & \ell ! x_{1} x_{2} \sum_{I \in\left(\begin{array}{l}
{[N] \backslash\{1,2\}} \\
\ell-2
\end{array}\right.} \prod_{i \in I} x_{i}+x_{1}^{k}+x_{2}^{k}-\left(x_{1}+x_{2}\right)^{k} \\
\geqslant & \ell ! x_{1} x_{2} \sum_{I \in\left(\begin{array}{c}
{[N] \backslash\{1,2\}} \\
\ell-2
\end{array}\right)} x_{2}^{\ell-2}+x_{1}^{k}+x_{2}^{k}-\left(x_{1}+x_{2}\right)^{k} \\
= & \ell ! x_{1} x_{2}^{\ell-1}\left(\begin{array}{c}
N-2 \\
\ell-2
\end{array}\right)-\sum_{v=1}^{k-1}\left(\begin{array}{l}
k \\
v
\end{array}\right) x_{1}^{v} x_{2}^{k-v} \\
\geqslant & \ell ! x_{1} x_{2}^{\ell-1}\left(\begin{array}{c}
N-2 \\
\ell-2
\end{array}\right)-\sum_{v=1}^{k-1}\left(\begin{array}{l}
k \\
v
\end{array}\right) x_{1} x_{2}^{k-1} \\
= & x_{1} x_{2}^{\ell-1}\left(\ell !\left(\begin{array}{c}
N-2 \\
\ell-2
\end{array}\right)-\left(2^{k}-2\right) x_{2}^{k-\ell}\right) .
\end{aligned}
$$

Como $x_{2}$ é o segundo menor valor dentre os $x_{i}$ 's, temos que $x_{2} \leqslant 2 / N$. Portanto, como $k \geqslant \ell$, temos

$$
\begin{aligned}
& q_{N, \mathrm{Id}_{\ell}}+\operatorname{Rev}_{k}\left(x_{1}, x_{2}, \ldots, x_{N}\right)-q_{N-1, \mathrm{Id}_{\ell}}+\operatorname{Rev}_{k}\left(x_{1}+x_{2}, x_{3}, x_{4}, \ldots, x_{N}\right) \\
& \geqslant x_{1} x_{2}^{\ell-1}\left(\ell !\left(\begin{array}{c}
N-2 \\
\ell-2
\end{array}\right)-\left(2^{k}-2\right)\left(\frac{2}{N}\right)^{k-\ell}\right) \text {. }
\end{aligned}
$$

Para mostrar que (3.12) é não negativo, consideramos três casos.

Caso $1(N \geqslant \ell+1)$. Temos $N \geqslant 4$. Portanto

$$
\begin{aligned}
\ell !\left(\begin{array}{c}
N-2 \\
\ell-2
\end{array}\right)-\left(2^{k}-2\right)\left(\frac{2}{N}\right)^{k-\ell} & \geqslant \ell !\left(\begin{array}{c}
N-2 \\
\ell-2
\end{array}\right)-\left(2^{k}-2\right) 2^{\ell-k} \\
& \geqslant \ell ! \frac{(N-\ell+1)^{\ell-2}}{(\ell-2) !}-2^{\ell} \\
& =\ell(\ell-1)(N-\ell+1)^{\ell-2}-2^{\ell},
\end{aligned}
$$

e como $\ell \geqslant 3$ e $N-\ell+1 \geqslant 2$, temos que (3.12) é não negativo.

Caso $2(N=\ell$ e $\ell \geqslant 4)$. Logo,

$$
\begin{aligned}
\ell !\left(\begin{array}{c}
N-2 \\
\ell-2
\end{array}\right)-\left(2^{k}-2\right)\left(\frac{2}{N}\right)^{k-\ell} & \geqslant \ell !\left(\begin{array}{c}
N-2 \\
\ell-2
\end{array}\right)-\left(2^{k}-2\right) 2^{\ell-k} \\
& =\ell !-2^{\ell}+2^{\ell-k+1}
\end{aligned}
$$

que é não negativo. 
Caso $3(N=\ell=3)$. Logo,

$$
\begin{aligned}
& q_{3, \operatorname{Id}_{3}+\operatorname{Rev}_{k}}\left(x_{1}, x_{2}, x_{3}\right)-q_{2, \operatorname{Id}_{3}, \operatorname{Rev}_{k}}\left(x_{1}+x_{2}, x_{3}\right) \\
& =3 ! x_{1} x_{2} x_{3}+x_{1}^{k}+x_{2}^{k}-\left(x_{1}+x_{2}\right)^{k} \\
& =6 x_{1} x_{2} x_{3}-\sum_{v=1}^{k-1}\left(\begin{array}{l}
k \\
v
\end{array}\right) x_{1}^{v} x_{2}^{k-v} \\
& =x_{1} x_{2}\left(6 x_{3}-k x_{1}^{k-2}-k x_{2}^{k-2}-\sum_{v=2}^{k-2}\left(\begin{array}{l}
k \\
v
\end{array}\right) x_{1}^{v-1} x_{2}^{k-v-1}\right) \\
& =x_{1} x_{2}\left(6 x_{3}-k x_{1}^{k-2}-k x_{2}^{k-2}-\sum_{v=1}^{k-3}\left(\begin{array}{c}
k \\
v+1
\end{array}\right) x_{1}^{v} x_{2}^{k-v-2}\right) \\
& =x_{1} x_{2}\left(6 x_{3}-k \sum_{v=0}^{k-2}\left(\begin{array}{c}
k-2 \\
v
\end{array}\right) x_{1}^{v} x_{2}^{k-2-v}+k \sum_{v=1}^{k-3}\left(\begin{array}{c}
k-2 \\
v
\end{array}\right) x_{1}^{v} x_{2}^{k-2-v}-\sum_{v=1}^{k-3}\left(\begin{array}{c}
k \\
v+1
\end{array}\right) x_{1}^{v} x_{2}^{k-2-v}\right) \\
& =x_{1} x_{2}\left(6 x_{3}-k\left(x_{1}+x_{2}\right)^{k-2}+\sum_{v=1}^{k-3}\left(k\left(\begin{array}{c}
k-2 \\
v
\end{array}\right)-\left(\begin{array}{c}
k \\
v+1
\end{array}\right)\right) x_{1}^{v} x_{2}^{k-v}\right) \text {, }
\end{aligned}
$$

onde na quarta igualdade, aplicamos a mudança de variável $v \rightarrow v+1$.

Vamos provar que este valor é não negativo. Como $x_{3}$ é o maior entre os $x_{i}$ 's, temos $x_{3} \geqslant 1 / 3$, portanto $x_{1}+x_{2} \leqslant 2 / 3$. Como $k(2 / 3)^{k-2}$ é uma função não crescente em $k$ quando $k \geqslant 2$, temos

$$
6 x_{3}-k\left(x_{1}+x_{2}\right)^{k-2} \geqslant 2-k\left(\frac{2}{3}\right)^{k-2} \geqslant 2-2\left(\frac{2}{3}\right)^{2-2}=0 .
$$

Resta provar que o somatório $\sum_{v=1}^{k-3}\left(k\left(\begin{array}{c}k-2 \\ v\end{array}\right)-\left(\begin{array}{c}k \\ v+1\end{array}\right)\right)$ é não negativo. Vamos provar um resultado levemente mais forte. Vamos mostrar que $k\left(\begin{array}{c}k-2 \\ v\end{array}\right)-\left(\begin{array}{c}k \\ v+1\end{array}\right) \geqslant 0$ para $k \geqslant 2$ e $1 \leqslant v \leqslant k-3$. Para isso, note que

$$
\begin{aligned}
k\left(\begin{array}{c}
k-2 \\
v
\end{array}\right)-\left(\begin{array}{c}
k \\
v+1
\end{array}\right) & =\frac{k(k-2) !}{(v+1) !(k-1-v) !}((v+1)(k-v-1)-(k-1)) \\
& =\frac{k(k-2) !}{(v+1) !(k-1-v) !}(v(k-v-2)) \\
& \geqslant \frac{k(k-2) !}{(v+1) !(k-1-v) !}(v(k-(k-3)-2)) \\
& \geqslant 0
\end{aligned}
$$

como queríamos mostrar.

Portanto, pelo Teorema 3.16, temos $z^{\prime}\left(\operatorname{Id}_{\ell}+\operatorname{Rev}_{k}\right)=\mathcal{U}_{\ell-1, \operatorname{Id}_{\ell}+\operatorname{Rev}_{k}}$. Agora, pela definição da Cota de Price para Minimização, temos

$$
\mathcal{U}_{\ell-1, \operatorname{Id}_{\ell}+\operatorname{Rev}_{k}}=\min \left\{\sum_{j=1}^{\ell-1} x_{i}^{k}: \sum_{j=1}^{\ell-1} x_{j}=1 \text { e } \forall j \in[\ell-1], x_{j} \geqslant 0\right\}=\frac{1}{(\ell-1)^{k-1}} .
$$




\section{Capítulo 4}

\section{Circuitos Hamiltonianos}

O trabalho apresentado neste capítulo foi realizado durante o estágio na Universidade de Hamburgo na Alemanha sob a supervisão do professor Mathias Schacht e orientação do Dr. Guilherme Mota e em parceria dos alunos de doutorado da Universidade de Hamburgo Jakob Schnitzer e Fabian Schulenburg e pode ser conferido em [4].

Neste capítulo, estudamos o problema de determinar qual a condição de grau mínimo que um hipergrafo $k$-uniforme $\mathcal{H}$ deve satisfazer para garantir a existência de um $\ell$-circuito Hamiltoniano. Este problema foi inicialmente estudado por Katona e Kierstead em [14]. Eles propuseram uma conjectura que foi confirmada pelo seguinte resultado de Rödl, Ruciński e Szemerédi [23, 24]: para todo $k \geq 3$, se $\mathcal{H}$ é um hipergrafo $k$-uniforme de ordem $n$ suficientemente grande que satisfaz $\delta_{k-1}(\mathcal{H}) \geqslant(1 / 2+o(1)) n$ então $\mathcal{H}$ contêm um $(k-1)$-circuito Hamiltoniano. Para isso, eles introduziram a técnica conhecida por método de absorção, que será discutida na Seção 4.1.

Em [18], Kühn e Osthus investigaram uma questão similar à conjecturada por Katona e Kierstead em [14] para 1-circuitos e provaram que todo hipergrafo 3-uniforme $\mathcal{H}$ que satisfaz $\delta_{2}(\mathcal{H}) \geqslant(1 / 4+o(1)) n$ contêm um 1-circuito Hamiltoniano. Esse resultado foi generalizado por Hàn e Schacht [9] (veja também [15]) para valores arbitrários de $k$ e para $\ell$-circuitos com $1 \leqslant \ell<k / 2$.

Teorema 4.1 (Hàn e Schacht [9]). Para todos os inteiros $k \geqslant 3$ e $1 \leqslant \ell<k / 2$ e todo $\gamma>0$, existe um $n_{0}$ tal que, para todo hipergrafo $k$-uniforme $\mathcal{H}$ de ordem $n \geqslant n_{0} \operatorname{com} n \in(k-\ell) \mathbb{N}$ e

$$
\delta_{k-1}(\mathcal{H}) \geqslant\left(\frac{1}{2(k-\ell)}+\gamma\right) n
$$

todo hipergrafo contêm um $\ell$-circuito Hamiltoniano.

Para ver a otimalidade assintótica da condição de grau mínimo no Teorema 4.1, consideramos o seguinte exemplo: sejam $\mathcal{H}_{k, \ell}=(V, E)$ um hipergrafo $k$-uniforme de ordem $n$ definido como a seguir. Seja $A \subset V$ um subconjunto de vértices de $\mathcal{H}_{k, \ell} \operatorname{com}|A|=\left\lceil\frac{n}{2(k-\ell)}-1\right\rceil$. Tomamos $E$ como o conjunto de todas as $k$-arestas que possuem pelo menos um vértice em $A$. Note que um $\ell$-circuito com $n$ vértices contêm $n /(k-\ell)$ arestas cada uma das quais contêm pelo menos um vértice de $A$ $\mathrm{e}$, para $\ell<k / 2$, todo vértice está contido em no máximo duas arestas do $\ell$-circuito. Portanto o hipergrafo $\mathcal{H}_{k, \ell}$ não contêm $\ell$-circuitos Hamiltonianos e $\delta_{k-1}\left(\mathcal{H}_{k, \ell}\right)=\left\lceil\frac{n}{2(k-\ell)}-1\right\rceil$. Em [10], Han e Zhao provaram uma versão do Teorema 4.1 que mostra que a condição sobre $\delta_{k-1}$ pode ser enfraquecida para $\delta_{k-1}(\mathcal{H}) \geqslant n /(2(k-\ell))$.

Kühn, Mycroft e Osthus [17] generalizaram o Teorema 4.1 para $1 \leqslant \ell<k-1$, resolvendo o 
problema de encontrar a condição justa para o $(k-1)$-grau mínimo que garante a existência de um $\ell$-circuito Hamiltoniano em hipergrafos $k$-uniformes. Fixado $d<k-1$, uma questão natural é saber qual condição devemos impor sobre $\delta_{d}$ para forçar a existência de um $\ell$-circuito Hamiltoniano. Nessa direção, em [5], Buß, Hàn e Schacht provaram a seguinte versão assintótica.

Teorema 4.2 (Buß, Hàn e Schacht [5]). Para todo $\gamma>0$ existe um $n_{0}$ tal que todo hipergrafo 3 -uniforme $\mathcal{H}$ de ordem $n \geqslant n_{0}$ com $n \in 2 \mathbb{N}$ e

$$
\delta_{1}(\mathcal{H}) \geqslant\left(\frac{7}{16}+\gamma\right)\left(\begin{array}{l}
n \\
2
\end{array}\right)
$$

contêm um 1-circuito Hamiltoniano.

Note que o hipergrafo $\mathcal{H}_{k, \ell}$ garante a otimalidade do resultado acima. Uma versão desse resultado sem $\gamma$ foi provada por Han e Zhao em [11]. Neste capítulo, generalizamos o Teorema 4.2 para hipergrafos $k$-uniformes e estabelecemos a cota inferior assintótica para $\delta_{k-2}$ que, para $1 \leqslant \ell<k / 2$, garante a existência de um $\ell$-circuito Hamiltoniano.

Teorema 4.3. Para todos os inteiros $k \geqslant 4$ e $1 \leqslant \ell<k / 2$ e todo $\gamma>0$, existe um $n_{0}$ tal que todo hipergrafo $k$-uniforme $\mathcal{H}=(V, E)$ de ordem $n \geqslant n_{0} \operatorname{com} n \in(k-\ell) \mathbb{N}$ e

$$
\delta_{k-2}(\mathcal{H}) \geqslant\left(\frac{4(k-\ell)-1}{4(k-\ell)^{2}}+\gamma\right)\left(\begin{array}{l}
n \\
2
\end{array}\right)
$$

contêm um $\ell$-circuito Hamiltoniano.

O hipergrafo $\mathcal{H}_{k, \ell}$ motiva a seguinte noção de extremalidade: sejam $k \geqslant 3$ e $\ell \geqslant 1$ inteiros e $0<\xi<1$. Um hipergrafo $k$-uniforme $\mathcal{H}=(V, E)$ é chamado de $(\ell, \xi)$-extremal se existe um conjunto $B \subset V$ tal que $|B|=\left\lfloor\frac{2(k-\ell)-1}{2(k-\ell)} n\right\rfloor$ e $e(B) \leqslant \xi\left(\begin{array}{l}n \\ k\end{array}\right)$.

Para provar uma versão exata do Teorema 4.2 (i.e., sem conter $\gamma$ na restrição do grau mínimo), Han e Zhao [11] dividiram sua prova em dois passos. O primeiro passo consiste em mostrar que ou o hipergrafo possui uma estrutura muito específica ou podemos fortalecer a condição de grau mínimo. Formalmente, o primeiro passo consiste em mostrar que dado, $\beta>0$, existe um $\gamma^{\prime}>0$ tal que todo hipergrafo $\mathcal{H}$ de ordem $n \in 2 \mathbb{N}$ suficientemente grande que não é $(1, \beta)$-extremal e que satisfaz $\delta_{1}(\mathcal{H}) \geqslant\left(\frac{7}{16}-\gamma^{\prime}\right)\left(\begin{array}{l}n \\ 2\end{array}\right)$ possui um 1-circuito Hamiltoniano. O segundo passo consiste em mostrar que existe um $\beta$ tal que todo hipergrafo $\mathcal{H}$ de ordem $n \in 2 \mathbb{N}$ suficientemente grande que é $(1, \beta)$-extremal e que satisfaz $\delta_{1}(\mathcal{H}) \geqslant \frac{7}{16}\left(\begin{array}{l}n \\ 2\end{array}\right)$ possui um 1-circuito Hamiltoniano. Seguindo essa ideia, provamos o seguinte resultado que implica o Teorema 4.3 (A prova disso será mostrada adiante).

Teorema 4.4. Para todos $0<\xi<1$ e inteiros $k \geqslant 4$ e $1 \leqslant \ell<k / 2$, existe um $\gamma>0$ e um inteiro $n_{0}$ tal que todo hipergrafo $k$-uniforme $\mathcal{H}$ de ordem $n>n_{0}$, com $n \in(k-\ell) \mathbb{N}$, que não é $(\ell, \xi)$-extremal e satisfaz

$$
\delta_{k-2}(\mathcal{H}) \geqslant\left(\frac{4(k-\ell)-1}{4(k-\ell)^{2}}-\gamma\right)\left(\begin{array}{l}
n \\
2
\end{array}\right)
$$

contêm um $\ell$-circuito Hamiltoniano.

Ressaltamos que para $k=3$ e $\ell=1$, a versão correspondente do Teorema 4.4 aparece como a parte não extremal do Teorema $4.2 \mathrm{em}$ [10].

Prova do Teorema 4.3. Sejam $1 \leqslant \ell<k / 2$ inteiros, com $k \geqslant 4$, e $\gamma>0$ fixo. Fixe uma constante 
$0<c<(2(k-\ell)-1) /(2(k-\ell))$. Pelo Teorema 4.4 aplicado para $k, \ell$ e $\xi=\gamma c^{k-2} / 2$, existem $\gamma^{\prime}>0$ e um inteiro $n_{0}^{\prime}$ tal que todo hipergrafo $k$-uniforme de ordem $n>n_{0}^{\prime} \operatorname{com} n \in(k-\ell) \mathbb{N}$ e que satisfaz (4.2) ou é $(\ell, \xi)$-extremal ou possui um $\ell$-circuito Hamiltoniano. Tome $n_{0}$ suficientemente grande e seja $\mathcal{H}$ um hipergrafo $k$-uniforme de ordem $n>n_{0} \operatorname{com} n \in(k-\ell) \mathbb{N}$ e

$$
\delta_{k-2}(\mathcal{H}) \geqslant\left(\frac{4(k-\ell)-1}{4(k-\ell)^{2}}+\gamma\right)\left(\begin{array}{l}
n \\
2
\end{array}\right)
$$

Note que, pelo Teorema 4.4, se $\mathcal{H}$ não é $(\ell, \xi)$-extremal então $\mathcal{H}$ possui um $\ell$-circuito Hamiltoniano. Para concluir a prova, mostraremos que $\mathcal{H}$ não é $(\ell, \xi)$-extremal e portanto $\mathcal{H}$ deve conter um $\ell$-circuito Hamiltoniano.

Dado um inteiro $n>0$, defina $b_{n}=\left\lfloor\frac{2(k-\ell)-1}{2(k-\ell)} n\right\rfloor$. Seja $B \subset V(\mathcal{H})$ um subconjunto arbitrário de vértices com $|B|=b_{n}$. Pela condição de grau mínimo sobre $\mathcal{H}$ temos que

$$
\begin{aligned}
e(\mathcal{H}[B]) & =\frac{1}{\left(\begin{array}{c}
k \\
2
\end{array}\right)} \sum_{F \in\left(\begin{array}{c}
B \\
k-2
\end{array}\right)} \operatorname{deg}_{\mathcal{H}[B]}(F) \\
& \geqslant \frac{1}{\left(\begin{array}{c}
k \\
2
\end{array}\right)} \sum_{F \in\left(\begin{array}{c}
B \\
k-2
\end{array}\right)}\left(\operatorname{deg}_{\mathcal{H}}(F)-\left(\begin{array}{c}
|V \backslash B| \\
2
\end{array}\right)-|V \backslash B||B|\right) \\
& =\frac{1}{\left(\begin{array}{c}
k \\
2
\end{array}\right)} \sum_{F \in\left(\begin{array}{c}
B \\
k-2
\end{array}\right)}\left(\operatorname{deg}_{\mathcal{H}}(F)-\left(\begin{array}{c}
n \\
2
\end{array}\right)+\left(\begin{array}{c}
b_{n} \\
2
\end{array}\right)\right) \\
& \geqslant \frac{1}{\left(\begin{array}{c}
k \\
2
\end{array}\right)} \sum_{F \in\left(\begin{array}{c}
B \\
k-2
\end{array}\right)}\left(\left(\frac{4(k-\ell)-1}{4(k-\ell)^{2}}+\gamma\right)\left(\begin{array}{c}
n \\
2
\end{array}\right)-\left(\begin{array}{c}
n \\
2
\end{array}\right)+\left(\begin{array}{c}
\left.\frac{2(k-\ell)-1}{2(k-\ell)} n-1\right) \\
2
\end{array}\right)\right) \\
& =\frac{1}{\left(\begin{array}{c}
k \\
2
\end{array}\right)} \sum_{F \in\left(\begin{array}{c}
B \\
k-2
\end{array}\right)}\left(\gamma\left(\begin{array}{l}
n \\
2
\end{array}\right)-\left(\left(\frac{2(k-\ell)-1}{2(k-\ell)}\right)^{2}\left(\begin{array}{c}
n \\
2
\end{array}\right)-\left(\begin{array}{c}
\frac{2(k-\ell)-1}{2(k-\ell)} n-1 \\
2
\end{array}\right)\right)\right. \\
& >\frac{\gamma}{2}\left(\begin{array}{c}
b_{n} \\
n
\end{array}\right)^{k-2}\left(\begin{array}{l}
n \\
k
\end{array}\right) \\
& >\xi\left(\begin{array}{l}
n \\
k
\end{array}\right),
\end{aligned}
$$

onde a última desigualdade segue da escolha de $\xi$. Portanto, $\mathcal{H}$ não é $(\ell, \xi)$-extremal e assim concluímos a prova.

Este capítulo está organizado da seguinte forma: na Seção 4.1 apresentamos a estratégia utilizada para provar o Teorema 4.4 e os principais lemas que são necessários para a utilização da técnica de absorção e na Seção 4.2 apresentamos a prova do Teorema 4.4.

\subsection{Ideia da Prova do Teorema Principal de Hipergrafos e Lemas Principais}

A prova do Teorema 4.4 utiliza o método de absorção introduzido por Rödl, Ruciński e Szemerédi em [23]. Para a utilização desse método são necessários os seguintes resultados que serão discutidos ao longo desta seção: Lema de Absorção (Lema 4.8), Lema do Reservatório (Lema 4.7) e Lema de Empacotamento por Caminhos (Lema 4.16). 
Dado $\beta>0$, dizemos que um $\ell$-caminho $\mathcal{P}$ em um hipergrafo $k$-uniforme $\mathcal{H}$ é $\beta$-absorvedor se para todo subconjunto $U \subset V(\mathcal{H})$, com $|U| \in(k-\ell) \mathbb{N}$, de tamanho no máximo $\beta$ n existe um $\ell$-caminho $\mathcal{Q}$ tal que $V(\mathcal{Q})=V(\mathcal{P}) \cup U$ e $\mathcal{Q}$ possui as mesmas extremidades de $\mathcal{P}$. Dizemos que um $\ell$-circuito em um hipergrafo $k$-uniforme de ordem $n>0$ é $\beta$-gerador se contêm pelo menos $(1-\beta) n$ vértices.

Dados $\eta>0$, o Lema de Absorção (Lema 4.8) garante a existência de um $\ell$-caminho $\beta$ absorvedor $\mathcal{P}$ em hipergrafos $k$-uniformes de ordem $n>0$ com $(k-2)$-grau mínimo pelo menos $\eta\left(\begin{array}{l}n \\ 2\end{array}\right)$. (Note que aqui ainda não necessitamos da condição de grau mínimo (4.2).) Portanto, o problema de encontrar um $\ell$-circuito Hamiltoniano em um hipergrafo $k$-uniforme se reduz a encontrar um $\ell$-circuito que é $\beta$-gerador e contêm $\mathcal{P}$.

Para obter um $\ell$-circuito $\beta$-gerador, encontramos primeiramente uma coleção limitada por uma cota superior, independente de $n$, de $\ell$-caminhos que cobre "quase" todos os vértices de $V(\mathcal{H}) \backslash \mathcal{P}$ e então conectamos esses $\ell$-caminhos em um único $\ell$-caminho utilizando vértices de um conjunto pequeno fixado previamente, conhecido como reservatório. O Lema do Reservatório (Lema 4.7) mostra que é possível encontrar um reservatório $R$ em um hipergrafo $k$-uniforme com $\delta_{k-2} \geqslant \eta\left(\begin{array}{l}n \\ 2\end{array}\right)$ tal que qualquer quantidade limitada de $\ell$-caminhos disjuntos pode ser conectado utilizando apenas vértices de $R$.

Podemos escolher os tamanhos de $\mathcal{P}$ e $R$ suficientemente pequenos de forma que o hipergrafo $\mathcal{H}[V \backslash(V(\mathcal{P}) \cup R)]$ satisfaça quase a mesma condição de grau mínimo que o hipergrafo $\mathcal{H}$. Dessa forma, o Lema de Cobertura por Caminhos (Lema 4.16) garante a existência de uma coleção de $\ell$-caminhos $\mathcal{S}$ que cobre quase todos os vértices de $V(\mathcal{H}) \backslash(\mathcal{P} \cup R)$. Essa é a única parte em que realmente necessitamos de todo o poder da condição de grau mínimo (4.2) em combinação com a não-extremalidade.

Os $\ell$-caminhos em $\mathcal{S}$ e o $\ell$-caminho $\mathcal{P}$ podem ser conectados, utilizando apenas vértices de $R$, para formar um $\ell$-circuito $\beta$-gerador que contêm $\mathcal{P}$. Como este $\ell$-circuito contêm quase todos os vértices de $\mathcal{H}$, a propriedade de absorção de $\mathcal{P}$ nos permite absorver os vértices que não estão no circuito, ou seja, os vértices que não estão nos $\ell$-caminhos ou não foram utilizados para conectar os $\ell$-caminhos. Dessa forma, obtemos o $\ell$-circuito Hamiltoniano desejado.

\subsubsection{Conexão e Reservatório}

Para construir um $\ell$-circuito $\beta$-gerador de um hipergrafo $k$-uniforme $\mathcal{H}$, primeiramente temos que achar uma família de $\ell$-caminhos e conectar suas extremidades. Para uma coleção $\left\{X_{i}, Y_{i}\right\}_{i \in[m]}$ de $2 m$ conjuntos mutuamente disjuntos de $\ell$ vértices, dizemos que um conjunto de $\ell$-caminhos $\mathcal{T}_{1}, \ldots, \mathcal{T}_{m}$ conecta $\left\{X_{i}, Y_{i}\right\}_{i \in[m]}$ se todos os $\ell$-caminhos são disjuntos nos vértices e, para todo $i \in[m]$, temos que $X_{i}$ e $Y_{i}$ são as extremidades de $\mathcal{T}_{i}$. O Lema 4.5 abaixo garante que é possível conectar uma coleção de $\ell$-caminhos disjuntos e, mais ainda, permite restringir as arestas utilizadas para conectar os $\ell$-caminhos a um subconjunto de vértices.

Lema 4.5 (Lema de Conexão). Sejam $k \geqslant 4$ e $m \geqslant 1$ inteiros e $\eta>0$. Defina $r_{0}=64 k m / \eta^{3}$. Sejam $\mathcal{H}=(V, E)$ um hipergrafo $k$-uniforme de ordem $n \geq r_{0},\left\{X_{i}, Y_{i}\right\}_{i \in[m]}$ uma coleção de $2 m$ conjuntos mutuamente disjuntos de $V$ com $\ell$ vértices e $R \subset V$ com $|R|=r \geqslant r_{0}$. Considere

$$
V^{\prime}=\left(\bigcup_{i \in[m]} X_{i} \cup Y_{i}\right) \cup R
$$


O seguinte vale para qualquer $1 \leqslant \ell<k / 2$.

Se $\left|N(K) \cap\left(\begin{array}{c}R \\ 2\end{array}\right)\right| \geqslant \eta\left(\begin{array}{c}r \\ 2\end{array}\right)$ para todo $K \in\left(\begin{array}{c}V^{\prime} \\ k-2\end{array}\right)$, então existem $\ell$-caminhos $\mathcal{T}_{1}, \ldots, \mathcal{T}_{m}$ de tamanho no máximo 4 que conectam $\left\{X_{i}, Y_{i}\right\}_{i \in[m]}$ e contêm apenas vértices de $V^{\prime}$.

Demonstração. Dados $k \geq 4$ e $m \geq 1$ inteiros e $\eta>0$, considere $r_{0}=64 k m / \eta^{3}$. Seja $\mathcal{H}$ um hipergrafo $k$-uniforme de ordem $n \geqslant r_{0},\left\{X_{i}, Y_{i}\right\}_{i \in[m]}$ uma coleção de $2 m$ conjuntos mutuamente disjuntos de $\ell$ vértices de $V$ e $R \subset V$ com $|R|=r \geqslant r_{0}$. Considere

$$
V^{\prime}=\left(\bigcup_{i \in[m]} X_{i} \cup Y_{i}\right) \cup R
$$

e suponha que $\left|N(K) \cap\left(\begin{array}{c}R \\ 2\end{array}\right)\right| \geqslant \eta\left(\begin{array}{l}r \\ 2\end{array}\right)$ para todo $K \in\left(\begin{array}{c}V^{\prime} \\ k-2\end{array}\right)$.

Fixe $1 \leq j \leq m$ e suponha que já construímos os $\ell$-caminhos $\mathcal{T}_{1}, \ldots, \mathcal{T}_{j-1}$ que conectam os pares $\left\{X_{i}, Y_{i}\right\}_{i \in[j-1]}$ usando somente vértices de $V^{\prime}$. Queremos construir o caminho $\mathcal{T}_{j}$ disjunto dos caminhos previamente construídos, disjunto dos conjuntos $\left\{X_{i}, Y_{i}\right\}_{i \in[m] \backslash\{j\}}$ e com extremidades $X_{j}$ e $Y_{j}$. Assim, defina $F_{j}=\bigcup_{i \in[m]}\left(X_{i} \cup Y_{i}\right) \cup \bigcup_{i \in[j-1]} V\left(\mathcal{T}_{i}\right)$ como o conjunto de vértices proibidos para $\mathcal{T}_{j}$. Em particular, pela definição de $r_{0}$, temos

$$
\left|F_{j}\right| \leqslant 4 k m \leqslant \frac{\eta r}{16}
$$

para todo $j \in[m]$.

A demonstração é dividida em dois casos. No primeiro caso, supomos que $k-2 \geqslant 2 \ell$ e consideramos a "folga" entre o tamanho do conjunto $X_{i} \cup Y_{i}$ e $(k-2)$ para algum $i \in[m]$. Essa folga nos permite utilizar a condição de grau mínimo diretamente sobre os conjuntos $X_{j}$ e $Y_{j}$ e encontrar os vértices restantes para completar a aresta que contêm $X_{j}$ e $Y_{j}$. No segundo caso, supomos que $k-1=2 \ell$. Observe que não podemos aplicar a condição de grau mínimo diretamente sobre os conjuntos $X_{j}$ e $Y_{j}$, pois para contornar essa situação, encontramos duas triplas $\left(x_{1}, L_{1}, y_{1}\right)$ e $\left(x_{2}, L_{2}, y_{2}\right)$ disjuntas com $x_{1}, x_{2}, y_{1}, y_{2} \in R \backslash F_{j}$ e $L_{1}, L_{2} \in\left(\begin{array}{c}R \backslash F_{j} \\ \ell-1\end{array}\right)$ que possuem a propriedade de que, para $i=1,2$, existem muitas arestas que contêm o conjunto $\left\{x_{i}\right\} \cup L_{i} \cup X_{j}$ e existem muitas arestas que contêm o conjunto $\left\{y_{i}\right\} \cup L_{i} \cup Y_{j}$. Dessa forma, utilizamos essas triplas para encontrar os vértices restantes e assim construir um caminho de tamanho 4 que conecta $X_{j}$ e $Y_{j}$ (ver Figura 4.1). A seguir apresentamos os detalhes sobre essa discussão.

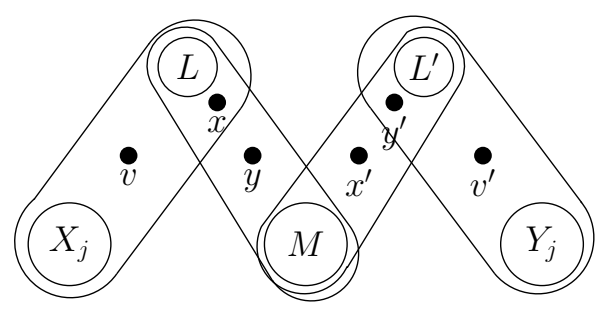

Figura 4.1: Caminho que conecta $X_{j}$ e $Y_{j}$

Primeiro, suponha que $k-2 \geqslant 2 \ell=\left|X_{j} \cup Y_{j}\right|$, então fixe um conjunto $Z$ de tamanho $k-2-2 \ell$ 
em $R \backslash F_{j}$. Pela escolha de $r_{0}$, sabemos que

$$
\begin{aligned}
\left|N\left(X_{j} \cup Y_{j} \cup Z\right) \cap\left(\begin{array}{c}
R \\
2
\end{array}\right)\right| & \geqslant \eta\left(\begin{array}{c}
|R| \\
2
\end{array}\right) \\
& \geqslant\left(\begin{array}{c}
|R| \\
2
\end{array}\right)-\left(\begin{array}{c}
\left(1-\frac{\eta}{2}\right)|R| \\
2
\end{array}\right) \\
& >\left(\begin{array}{c}
|R| \\
2
\end{array}\right)-\left(\begin{array}{c}
r-4 k m \\
2
\end{array}\right) \\
& \geqslant\left(\begin{array}{c}
|R| \\
2
\end{array}\right)-\left(\begin{array}{c}
\left|R \backslash F_{j}\right| \\
2
\end{array}\right) \\
& >0 .
\end{aligned}
$$

Assim, basta escolher uma aresta $X_{j} \cup Y_{j} \cup Z \cup Z^{\prime} \operatorname{com} Z^{\prime} \subset R \backslash F_{j}$ para ser o $\ell$-caminho $\mathcal{T}_{j}$ desejado.

Agora suponha que $2 \ell=k-1$. Para isso, utilizamos o seguinte fato. Sejam $L \in\left(\begin{array}{c}R \backslash F_{j} \\ \ell-1\end{array}\right)$ e $x, y \in R \backslash F_{j} \operatorname{com} x \neq y$. Dizemos que $(x, L, y)$ é uma tripla extensora de $R \backslash F_{j}$ se

$$
\left|N_{R \backslash F_{j}}\left(X_{j} \cup L \cup\{x\}\right)\right| \geqslant \eta r / 4 \text { e }\left|N_{R \backslash F_{j}}\left(Y_{j} \cup L \cup\{y\}\right)\right| \geqslant \eta r / 4 .
$$

Fixe $L \in\left(\begin{array}{c}R \backslash F_{j} \\ \ell-1\end{array}\right)$. A Afirmação 4.6 mostra que um conjunto $L \in\left(\begin{array}{c}R \backslash F_{j} \\ \ell-1\end{array}\right)$ está contido em muitas triplas extensoras.

Afirmação 4.6. Para todo $L \in\left(\begin{array}{c}R \backslash F_{j} \\ \ell-1\end{array}\right)$ existem pelo menos $(\eta r / 8)^{2}$ escolhas de pares de vértices $x, y \in R \backslash F_{j}$ tais que $(x, L, y)$ é uma tripla extensora.

Demonstração. Dado $L \in\left(\begin{array}{c}R \backslash F_{j} \\ \ell-1\end{array}\right)$, considere

$$
A_{X_{j}}=\left\{x \in R:\left|N\left(X_{j} \cup L \cup\{x\}\right) \cap R\right| \geqslant \eta r / 4+\eta r / 16\right\}
$$

e seja $a_{x}=\left|A_{X_{j}}\right|$. Dessa forma, temos

$$
\begin{aligned}
\eta\left(\begin{array}{l}
r \\
2
\end{array}\right) & \leqslant\left|N\left(X_{j} \cup L\right) \cap\left(\begin{array}{c}
R \\
2
\end{array}\right)\right| \\
& =\frac{1}{2}\left(\sum_{x \in A_{X_{j}}}\left|N\left(X_{j} \cup L \cup\{x\}\right) \cap R\right|+\sum_{x \notin A_{X_{j}}}\left|N\left(X_{j} \cup L \cup\{x\}\right) \cap R\right|\right) \\
& <\frac{1}{2}\left(a_{x} r+\left(r-a_{x}\right) \frac{5 \eta r}{16}\right) .
\end{aligned}
$$

Logo, $a_{x} \geqslant 3 \eta r / 4 \geqslant \eta r / 4+\eta r / 16$. Consequentemente, por (4.3), existem pelo menos $a_{x}-\left|F_{j}\right| \geqslant \eta r / 4$ vértices $x \in A_{X_{j}} \backslash F_{j}$ tais que $\left|N\left(X_{j} \cup L \cup\{x\}\right) \cap\left(R \backslash F_{j}\right)\right| \geqslant \eta r / 4$. Analogamente, existem pelo menos $\eta r / 4$ vértices $y \in R \backslash F_{j}$ tal que $\left|N\left(Y_{j} \cup L \cup\{x\}\right) \cap\left(R \backslash F_{j}\right)\right| \geqslant \eta r / 4$. Assim, existem pelo menos $\left(\begin{array}{c}\eta r / 4 \\ 2\end{array}\right)>(\eta r / 8)^{2}$ escolhas para pares $x, y \in V \backslash F_{j}$ tais que $(x, L, y)$ é uma tripla extensora.

Considere $S \in\left(\begin{array}{c}R \backslash F_{j} \\ \ell-2\end{array}\right)$. Para toda tripla extensora $(x, L, y)$ com $S \cap(L \cup\{x, y\})=\emptyset$ temos, pela condição de grau mínimo (4.1) aplicada a $S \cup L \cup\{x, y\}$, que existem pelo menos

$$
\eta\left(\begin{array}{c}
|R| \\
2
\end{array}\right)-\left(\begin{array}{c}
\left|F_{j}\right| \\
2
\end{array}\right)-\left|F_{j}\right| r \geqslant(\eta / 2)\left(\begin{array}{c}
\left|R \backslash F_{j}\right| \\
2
\end{array}\right)
$$


pares $M \in\left(\begin{array}{c}R \backslash F_{j} \\ 2\end{array}\right)$ tais que $S \cup M \cup L \cup\{x, y\}$ é uma aresta de $\mathcal{H}$. Como isso é válido para toda tripla extensora e existem pelo menos $\left(\begin{array}{c}R \backslash F_{j} \\ \ell-1\end{array}\right)(\eta r / 8)^{2}$ triplas extensoras, então existe um conjunto $M \in\left(\begin{array}{c}R \backslash F_{j} \\ 2\end{array}\right)$ que forma uma aresta em $\mathcal{H}$ juntamente com pelo menos $(\eta / 2)(\eta r / 8)^{2}\left(\begin{array}{c}\left|R \backslash F_{j}\right| \\ \ell-1\end{array}\right)$ triplas extensoras. Como $r \geqslant 64 \mathrm{~km} / \eta^{3}$, isso é mais que a quantidade máxima de triplas que intersectam uma tripla fixa. Portanto, existem duas triplas extensoras completamente disjuntas $(x, L, y) \mathrm{e}$ $\left(x^{\prime}, L^{\prime}, y^{\prime}\right)$ que formam uma aresta em $\mathcal{H}$ juntamente com $M^{\prime}=M \cup S$.

Pela definição de triplas extensoras, temos

$$
\left|N_{R \backslash F_{j}}\left(X_{j} \cup L \cup\{x\}\right)\right| \geqslant \eta r / 4>k+1=\left|M^{\prime} \cup L^{\prime} \cup\left\{x^{\prime}, y^{\prime}, y\right\}\right|
$$

$\mathrm{e}$

$$
\left.\left|N_{R \backslash F_{j}}\left(Y_{j} \cup L^{\prime} \cup\left\{y^{\prime}\right\}\right)\right| \geqslant \eta r / 4>k+2=\mid M^{\prime} \cup L \cup\left\{x, y, x^{\prime}\right\}\right) \mid+1 .
$$

Consequentemente, existem vértices $v, v^{\prime} \in R \backslash F_{j}$ tais que as arestas

$$
\left\{X_{j} \cup L \cup\{v, x\}\right\},\left\{M^{\prime} \cup L \cup\{x, y\}\right\},\left\{M^{\prime} \cup L^{\prime} \cup\left\{x^{\prime}, y^{\prime}\right\}\right\} \text { e }\left\{Y_{j} \cup L^{\prime} \cup\left\{y^{\prime}, v^{\prime}\right\}\right\}
$$

formam um caminho de tamanho 4 que conecta $X_{j}$ e $Y_{j}$.

Na prova do Teorema 4.4, inicialmente encontramos uma família de $\ell$-caminhos que cobre quase todos os vértices do hipergrafo e desejamos conectar esses $\ell$-caminhos para formar um $\ell$-circuito $\beta$-gerador. O Lema do Reservatório abaixo garante a existência de um conjunto pequeno $R$ tal que podemos conectar uma coleção arbitrária de $\ell$-conjuntos com no máximo $2 m$ elementos, usando apenas vértices de $R$.

Lema 4.7 (Lema do Reservatório). Sejam $\eta, \varepsilon>0$ fixos e $k, m \in \mathbb{N}$ inteiros positivos com $k \geqslant 4$ e $m \geqslant 1$. Então existe $n_{0}>0$ tal que, para todo hipergrafo $k$-uniforme $\mathcal{H}$ de ordem $n \geqslant n_{0}$ com $\delta_{k-2}(\mathcal{H}) \geqslant \eta\left(\begin{array}{l}n \\ 2\end{array}\right)$, existe um conjunto $R \subset V$, com $|R| \leqslant \varepsilon n$, tal que o seguinte vale para todo $1 \leqslant \ell<k / 2$.

Dado $j \leqslant m$, para toda coleção $\left\{X_{i}, Y_{i}\right\}_{i \in[j]}$ de $2 j$ conjuntos mutuamente disjuntos com $\ell$ vértices existem $\ell$-caminhos $\mathcal{T}_{1}, \ldots, \mathcal{T}_{j}$ de tamanho no máximo 4 que conectam $\left\{X_{i}, Y_{i}\right\}_{i \in[j]}$ e contêm somente vértices de $\bigcup_{i \in[j]}\left(X_{i} \cup Y_{i}\right) \cup R$.

Demonstração. A ideia geral consiste em mostrar que se escolhermos $0<p<1$ adequadamente e gerarmos um conjunto $R$ de vértices de forma que adicionamos cada vértice de $V$ a $R$ com probabilidade $p$ então é possível obter, com alta probabilidade, um conjunto que satisfaz as condições do lema.

Sejam $\eta, \varepsilon>0$ fixos e $k, m, \ell \in \mathbb{N}$ inteiros positivos com $k \geq 4$ e $1 \leqslant \ell<k / 2$. Fixe um inteiro $n_{0}$ suficientemente grande e seja $\mathcal{H}=(V, E)$ um hipergrafo $k$-uniforme de ordem $|V|=n \geqslant n_{0}$ com $\delta_{k-2}(\mathcal{H}) \geqslant \eta\left(\begin{array}{c}n \\ 2\end{array}\right)$. Tome $p=\varepsilon(1-\eta)$ e, dado $F \in\left(\begin{array}{c}V(\mathcal{H}) \\ k-2\end{array}\right)$, denote por $G_{F}$ o grafo link de $F$. Note que o conjunto de arestas de um grafo completo com $n$ vértices pode ser decomposto em no máximo $n$ emparelhamentos disjuntos. Dessa forma, denotamos por $M_{1}(F), \ldots, M_{i_{0}}(F)$ uma decomposição das arestas de $G_{F}$ em emparelhamentos disjuntos, onde $i_{0}<n$.

Seja $V_{p} \subset V(\mathcal{H})$ um conjunto gerado aleatoriamente onde para cada vértice $v \in V$ adicionamos 
$v$ a $V_{p}$ independentemente com probabilidade $p$. Para todo $i \in\left[i_{0}\right]$, denote por

$$
X_{i}(F)=\left|M_{i}(F) \cap\left(\begin{array}{c}
V_{p} \\
2
\end{array}\right)\right|
$$

a variável aleatória que indica quantas arestas do emparelhamento $M_{i}(F)$ foram adicionadas ao grafo $G_{F}\left[V_{p}\right]$. Dessa forma, aplicando a Desigualdade de Chernoff (Teorema 2.2), temos que

$$
\frac{\varepsilon n}{2} \leqslant\left|V_{p}\right| \leqslant p n+(k n \ln 20)^{1 / 2} \leqslant \varepsilon n-2 m \ell
$$

ocorre com probabilidade pelo menos $9 / 10$.

Pela Desigualdade de Chernoff (Teorema 2.2) e do fato de que $\left|M_{i}(F)\right| \leqslant n / 2$, temos que, com probabilidade pelo menos $1-1 / n^{k-1}$,

$$
X_{i}(F) \geqslant\left|M_{i}(F)\right| p^{2}-(k n \ln n)^{1 / 2}
$$

para todo $i \in\left[i_{0}\right]$. Dessa forma, usando o fato de que $\sum_{i \in\left[i_{0}\right]}\left|M_{i}(F)\right|=\operatorname{deg}_{\mathcal{H}}(F)$, temos

$$
\operatorname{deg}_{V_{p}}(F)=\sum_{i \in\left[i_{0}\right]} X_{i}(F) \geqslant p^{2} \operatorname{deg}_{\mathcal{H}}(F)-n(k n \ln n)^{1 / 2}
$$

com probabilidade pelo menos $1-1 / n^{k-1}$. Assim, repetindo o argumento acima para todo conjunto $F \in\left(\begin{array}{c}V \\ k-2\end{array}\right)$ e usando a cota da união, temos que (4.5) é válida para todo $F \in\left(\begin{array}{c}V \\ k-2\end{array}\right)$ com probabilidade pelo menos $(1-1 / n)$. Portanto, com probabilidade positiva, podemos obter um conjunto $R$ que satisfaz (4.4) e (4.5) para todo $F \in\left(\begin{array}{c}V \\ k-2\end{array}\right)$.

Seja $\mathcal{S}=\left\{X_{i}, Y_{i}\right\}_{i \in[m]}$ uma coleção de $2 m$ conjuntos mutuamente disjuntos de $\ell$ vértices. Denote por $S=\bigcup_{i=1}^{m} X_{i} \cup Y_{i}$. Portanto, por (4.4), temos que $|R \cup S| \leqslant \varepsilon n$ e

$$
\begin{aligned}
\operatorname{deg}_{R \cup S}(F) & \geq \operatorname{deg}_{R}(F) \\
& \geq \varepsilon^{2}(1-\varepsilon)^{2} \eta\left(\begin{array}{l}
n \\
2
\end{array}\right)-n(k n \ln n)^{1 / 2} \\
& \geq \frac{\varepsilon^{2} \eta(1-\varepsilon)^{2}}{2}\left(\begin{array}{l}
n \\
2
\end{array}\right)
\end{aligned}
$$

para todo $F \in\left(\begin{array}{c}V \\ k-2\end{array}\right)$. Dessa forma, aplicando o Lema 4.5 em $\mathcal{H}[R \cup S] \operatorname{com} \eta^{\prime}=\varepsilon^{2} \eta(1-\gamma)^{2}$, podemos conectar $X_{i}$ e $Y_{i}$ utilizando apenas vértices de $R$.

\subsubsection{Absorção}

Dado um hipergrafo $k$-uniforme $\mathcal{H}$ e um subconjunto $U \subset V$ com $|U| \in(k-\ell) \mathbb{N}$, dizemos que um $\ell$-caminho $\mathcal{P}$ absorve $U$ se existe um $\ell$-caminho $\mathcal{Q}$ com as mesmas extremidades de $\mathcal{P}$ e $V(\mathcal{Q})=V(\mathcal{P}) \cup U$. Lembre que um $\ell$-caminho $\mathcal{P}$ é $\varepsilon$-absorvedor se absorve qualquer subconjunto $U \subset V$ de vértices com $|U| \in(k-\ell) \mathbb{N}$ e $|U| \leq \beta n$. No final da prova do Teorema 4.4, utilizamos a propriedade do caminho $\varepsilon$-absorvedor para absorver os vértices que não fazem parte do $\ell$-circuito $\beta$ gerador e dessa forma obter um $\ell$-circuito Hamiltoniano. A existência de um caminho $\varepsilon$-absorvedor é garantida pelo Lema 4.8 abaixo.

Lema 4.8 (Lema de Absorção). Para todos $\gamma, \zeta>0$ e inteiros $k \geqslant 4$ e $1 \leqslant \ell<k / 2$ existe $\varepsilon>0$ tal 
que o seguinte vale para todo $n$ suficientemente grande. Seja $\mathcal{H}=(V, E)$ um hipergrafo $k$-uniforme de ordem $n$ com

$$
\delta_{k-2}(\mathcal{H}) \geqslant \gamma\left(\begin{array}{l}
n \\
2
\end{array}\right)
$$

Então existe um $\ell$-caminho $\varepsilon$-absorvedor $\mathcal{A} \operatorname{com}|V(\mathcal{A})| \leqslant \zeta n$.

Demonstração. A demonstração consiste em três passos. No primeiro passo, mostramos que dado um conjunto $S$ com $k-\ell$ vértices, existem "muitos" caminhos de tamanho 3 que absorvem $S$. No segundo passo, encontramos uma família $\mathcal{F}$ "pequena" de caminhos de tamanho 3 tal que, para qualquer conjunto $S$ com $k-\ell$ vértices, existe um caminho em $\mathcal{F}$ que absorve $S$. No terceiro passo, utilizamos o Lema 4.5 para conectar os caminhos de $\mathcal{F}$ em um único caminho, obtendo assim o caminho desejado.

Fixe $\gamma, \zeta>0$ e inteiros $k \geqslant 4$ e $1 \leqslant \ell<k / 2$. Considere as seguintes constantes auxiliares

$$
\widetilde{\gamma}=\gamma / 4 k !, \quad t=3 k-2 \ell \quad \text { e } \quad \varepsilon=\frac{\zeta \widetilde{\gamma}^{10}}{100 k}
$$

Considere $n$ suficientemente grande e seja $\mathcal{H}=(V, E)$ um hipergrafo $k$-uniforme de ordem $n$ com $\delta_{k-2}(\mathcal{H}) \geqslant \gamma\left(\begin{array}{l}n \\ 2\end{array}\right)$. Dados dois conjuntos disjuntos de vértices $A, B \subset V(\mathcal{H}) \operatorname{com}|A| \leqslant k-2$ e $|B| \leqslant t+k$, por $(4.6)$, temos

$$
\operatorname{deg}_{\mathcal{H}[V \backslash B]}(A) \geqslant \frac{(n-|A|) \cdots(n-k+3)}{(k-|A|) !} \cdot \gamma\left(\begin{array}{l}
n \\
2
\end{array}\right)-|B| n^{k-|A|-1} \geqslant \widetilde{\gamma} n^{k-|A|} .
$$

A Afirmação 4.9 abaixo implica que, para qualquer $S \in\left(\begin{array}{c}V \\ k-\ell\end{array}\right)$, existem $\Omega\left(n^{t}\right) \ell$-caminhos de tamanho 3 que absorvem $S$.

Afirmação 4.9. Para todo $S \in\left(\begin{array}{c}V \\ k-\ell\end{array}\right)$ existem pelo menos $\widetilde{\gamma}^{5} n^{t} \ell$-caminhos de tamanho 3 que absorvem $S$.

Demonstração. Seja $S_{1} \cup S_{2}=S$ subconjuntos de $S$ tal que

$$
\left|S_{1}\right| \geqslant\left|S_{2}\right| \geqslant\left|S_{1}\right|-1 \quad \text { e } \max \{0, \ell-(k-2 \ell)\} \leqslant\left|S_{1} \cap S_{2}\right|<\ell
$$

e sejam $s_{1}=\left|S_{1}\right|, s_{2}=\left|S_{2}\right|$ e $s_{3}=\left|S_{1} \cap S_{2}\right|$. Assim, temos

$$
s_{1}+s_{2}-s_{3}=|S|=k-\ell .
$$

Em particular, por (4.8), temos $s_{1}+s_{2}-s_{3} \leqslant 2 s_{2}+1-(\ell-(k-2 \ell))$. Portanto, por (4.9), concluímos que $s_{2} \geqslant \ell$.

Estamos interessados em encontrar vários caminhos de tamanho 3 que absorvem $S$. Note que se um caminho $\mathcal{P}$ de tamanho 3 absorve $S$ então o hipergrafo induzido por $V(\mathcal{P}) \cup S$ possui uma estrutura similar à estrutura da Figura 4.2. 


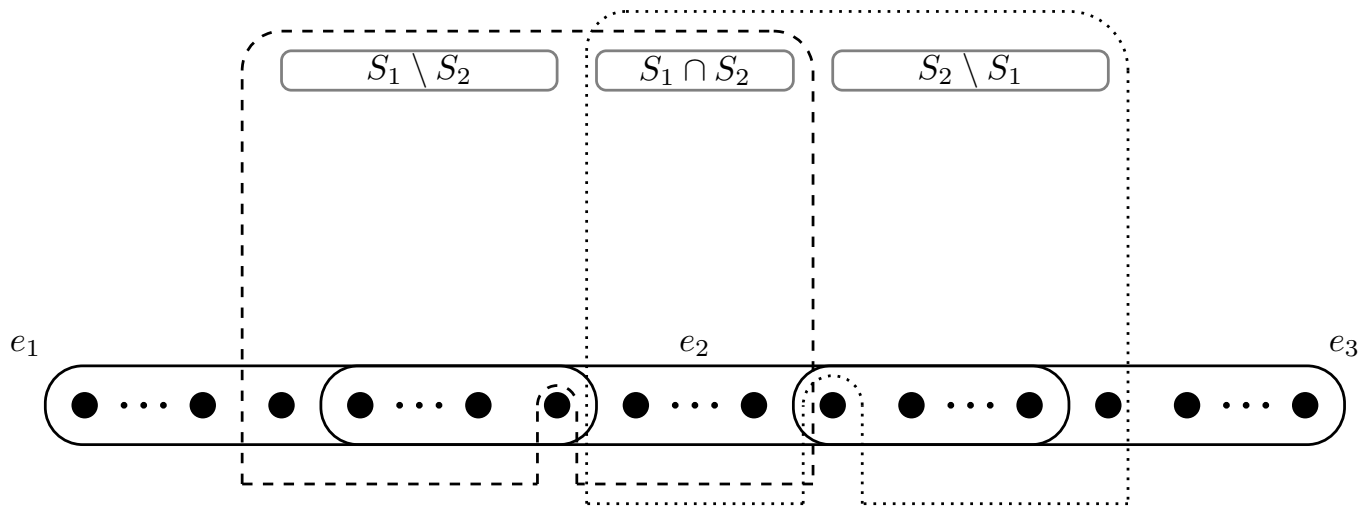

Figura 4.2: Hipergrafo induzido que absorve $S_{1} \cup S_{2}$

A seguir, mostramos uma ordem de escolha dos vértices de um caminho que absorve $S$. Em cada passo selecionamos somente conjuntos de vértices que são disjuntos de $S$ e de todos os vértices selecionados nos passos anteriores, de forma que tais conjuntos satisfazem a estrutura do hipergrafo induzido similar à Figura 4.2.

i) Como $s_{1} \leqslant k-\ell-1 \leqslant k-2$, por (4.7), existem $\widetilde{\gamma} n^{k-s_{1}}$ escolhas para um conjunto $X$ de tamanho $\left(k-s_{1}\right)$ tal que $f_{1}=X \dot{\cup} S_{1}$ é uma aresta de $\mathcal{H}$. Usando $(4.8)$ e (4.9), temos que

$$
\begin{aligned}
k-\ell-\left(\ell-s_{3}\right)-s_{1} & =k-2 \ell-\left(s_{1}-s_{3}\right) \\
& =k-2 \ell-\left(k-\ell-s_{2}\right) \\
& =s_{2}-\ell \\
& \geqslant 0 .
\end{aligned}
$$

Dessa forma, seja $L_{1} \dot{\cup} F \dot{\cup} F_{1}$ uma partição do conjunto $X$ tal que $\left|L_{1}\right|=\ell,|F|=\ell-s_{3}$ e $\left|F_{1}\right|=k-\left|L_{1} \dot{\cup} F \dot{\cup} S_{1}\right|$.

ii) Como $k \geqslant 4$, temos que $s_{1} \geqslant 2$ e $s_{2}+\left(\ell-s_{3}\right)=k-s_{1} \leqslant k-2$. Portanto, por (4.7), existem $\widetilde{\gamma} n^{k-s_{2}-\left(\ell-s_{3}\right)}$ escolhas para um conjunto $Y$ tais que $f_{2}=S_{2} \dot{\cup} F \dot{\cup} Y$ é uma aresta de $\mathcal{H}$. Como $k-\ell-\left(\ell-s_{3}\right)-s_{2} \geq\left|F_{1}\right| \geq 0$, seja $L_{2} \cup \dot{\cup} F_{2}$ uma partição de $Y$ tal que $\left|L_{2}\right|=\ell$ e $\left|F_{2}\right|=k-\left|L_{2} \dot{\cup} F \dot{\cup} S_{2}\right|$.

iii) Fixe $L_{1}^{\prime} \subset L_{1}$ e $L_{2}^{\prime} \subset L_{2}$ subconjuntos de tamanho $\ell-1$. Note que

$$
\left|L_{1}^{\prime} \dot{\cup} L_{2}^{\prime} \dot{\cup} F \dot{\cup} F_{1} \dot{\cup} F_{2}\right|=k-2=|X|+|Y|-2 .
$$

Portanto, existem pelo menos $\widetilde{\gamma} n^{2}$ escolhas para um par de vértices $\left\{x_{1}, x_{2}\right\}$ tal que

$$
e_{2}=\left\{x_{1}, x_{2}\right\} \dot{\cup} L_{1}^{\prime} \dot{\cup} L_{2}^{\prime} \dot{\cup} F \dot{\cup} F_{1} \dot{\cup} F_{2}
$$

é uma aresta em $\mathcal{H}$.

iv) Como $k \geqslant 4$, temos $\ell+1 \leqslant k-2$. Portanto, existem $\widetilde{\gamma} n^{k-(\ell+1)}$ escolhas disjuntas de $e_{2}, f_{1}$ e $f_{2}$ para as arestas $e_{1}$ e $e_{3}$ tal que $\left\{x_{1}\right\} \dot{\cup} L_{1} \subset e_{1}$ e $\left\{x_{2}\right\} \dot{\cup} L_{2} \subset e_{3}$. 
Por construção, temos

$$
e_{1} \cap e_{2}=\left\{x_{1}\right\} \dot{\cup} L_{1}^{\prime} \quad \text { e } \quad e_{2} \cap e_{3}=\left\{x_{2}\right\} \dot{\cup} L_{2}^{\prime} .
$$

Portanto, as arestas $e_{1}, e_{2}$ e $e_{3}$ formam um $\ell$-caminho $\mathcal{P}$ em $\mathcal{H}$. Mais ainda, como

$$
e_{1} \cap f_{1}=L_{1}, \quad\left|f_{1} \cap f_{2}\right|=\left|\left(S_{1} \cap S_{2}\right) \cup F\right| \stackrel{\text { i) }}{=} \ell \quad \text { e } \quad f_{2} \cap e_{3}=L_{2},
$$

as arestas $e_{1}, f_{1}, f_{2}$ e $e_{3}$ formam um $\ell$-caminho $\mathcal{P}^{\prime}$. Como $k-\ell-1 \geqslant \ell$, podemos selecionar para $\mathcal{P}$ e $\mathcal{P}^{\prime}$ as mesmas extremidades em $e_{1}$ e $e_{3}$. Além disso, $V\left(\mathcal{P}^{\prime}\right)=V(\mathcal{P}) \cup S$ e, portanto, o $\ell$-caminho $\mathcal{P}$ absorve $S$. Por i)-iv) temos que existem pelo menos

$$
\widetilde{\gamma} n^{k-s_{1}} \cdot \widetilde{\gamma} n^{k-s_{2}-\left(\ell-s_{3}\right)} \cdot \widetilde{\gamma} n^{2} \cdot\left(\widetilde{\gamma} n^{k-(\ell+1)}\right)^{2}=\widetilde{\gamma}^{5} n^{t}
$$

escolhas para $\mathcal{P}$.

Seguindo a ideia apresentada em [23], seja $\mathcal{F} \subset V(\mathcal{H})^{t}$ uma família de $t$-conjuntos ordenados de vértices selecionados de $V(\mathcal{H})^{t}$ independentemente com probabilidade

$$
p=\frac{4 \varepsilon}{\widetilde{\gamma}^{5}} n^{-t+1}
$$

Um $\ell$-caminho em $V(\mathcal{H})^{t}$ é um conjunto ordenado $\left(v_{1}, \ldots, v_{t}\right)$ de vértices tal que

$$
e_{1}=\left\{v_{1}, \ldots, v_{k}\right\}, \quad e_{2}=\left\{v_{k-\ell+1}, \ldots, v_{2 k-\ell}\right\} \quad \text { e } \quad e_{3}=\left\{v_{2 k-2 \ell+1}, \ldots, v_{t}\right\}
$$

são arestas em $\mathcal{H}$. Dessa forma, temos:

I) Pela Desigualdade de Chernoff (Teorema 2.2), com alta probabilidade, temos

$$
|\mathcal{F}| \leqslant 2 p n^{t}
$$

II) Pela Afirmação 4.9, para cada conjunto $S$ de tamanho $k-\ell$, pelo menos $\widetilde{\gamma}^{5} n^{t} \ell$-caminhos em $V(\mathcal{H})^{t}$ absorvem $S$. Dessa forma, pela Desigualdade de Chernoff (Teorema 2.2), com alta probabilidade, para todo $S \in\left(\begin{array}{c}V \\ k-\ell\end{array}\right)$ existem pelo menos $2 \varepsilon n$ caminhos de tamanho $\ell$ em $\mathcal{F}$ que absorvem $S$.

III) Pela Desigualdade de Markov (Teorema 2.3), com probabilidade pelo menos $1 / 2$, a quantidade de pares intersectantes de $t$-conjuntos em $\mathcal{F}$ é no máximo $\varepsilon n$.

Seja $\mathcal{F}$ uma família que satisfaz as condições I)-III). Para cada um dos pares que se intersectam em $\mathcal{F}$, deletamos um dos $t$-conjuntos. Seja $\mathcal{F}^{\prime} \subset \mathcal{F}$ a família dos conjuntos restantes. Usando o Lema 4.5, com $m$ sendo a quantidade de $\ell$-caminhos em $\mathcal{F}^{\prime}$ e $R=V(\mathcal{H})$, podemos conectar todos os $\ell$-caminhos em $\mathcal{F}^{\prime}$ em um $\ell$-caminho $\mathcal{A}$ com

$$
|V(\mathcal{A})| \leqslant\left|\mathcal{F}^{\prime}\right| \cdot(4 k+t) \leqslant 2 p n^{t} \cdot 7 k=\frac{56 k}{\widetilde{\gamma}^{5}} \varepsilon n \leqslant \zeta n
$$

e este caminho é $\varepsilon$-absorvedor. 


\subsubsection{Empacotamento dos Vértices de $\mathcal{H}$}

Nesta seção, encontramos uma família de $\ell$-caminhos disjuntos que cobre "quase todos" os vértices de $\mathcal{H}$. Para isso, utilizamos uma extensão do Lema da Regularidade de Szemerédi [26] para hipergrafos, discutida na Seção 2.2.2. Primeiro, aplicamos o Lema da Regularidade Fraca em $\mathcal{H}$ e obtemos um hipergrafo reduzido $\mathcal{R}$ que satisfaz "quase" a mesma condição de grau mínimo que $\mathcal{H}$. Utilizando a condição de grau mínimo do hipergrafo reduzido $\mathcal{R}$, encontramos um empacotamento fracionário de $\mathcal{R}$ por $2 \ell$-caminhos de comprimento 2 que cobre "quase" todos os vértices de $\mathcal{R}$. Finalmente, utilizando o Lema 4.15 (ver Lemma 2.7 [10]) e o empacotamento fracionário de $\mathcal{R}$, obtemos uma família de $\ell$-caminhos disjuntos em $\mathcal{H}$, como desejado. Denotamos por $\mathcal{C}_{\ell}$ o $2 \ell$-caminho de tamanho 2 com vértices em $[2 k-2 \ell]$ e arestas $\{1, \ldots, k\}$ e $\{k-2 \ell+1, \ldots, 2 k-2 \ell\}$.

Definição 4.10. Sejam $\mathcal{C}$ e $\mathcal{R}$ hipergrafos $k$-uniformes, $\beta>0$, e seja $\Phi$ uma coleção de homomorfismos em hipergrafos de $\mathcal{C}$ em $\mathcal{R}$. Uma função $h: \Phi \rightarrow\left\{a \beta: a \in \mathbb{N}_{>0}\right\}$ é chamada $\beta$-hom $(\mathcal{C})$ empacotamento se

$$
w_{h}(v)=\sum_{u \in V(\mathcal{C})} \sum_{\varphi \in \Phi: v=\varphi(u)} h(\varphi) \leq 1
$$

para todo $v \in V(\mathcal{R})$. Denotamos por

$$
w(h)=\sum_{v \in V(\mathcal{R})} w_{h}(v)=\sum_{\varphi \in \Phi} h(\varphi)|V(\mathcal{C})|
$$

o peso do empacotamento.

O Exemplo 4.11 abaixo sugere uma forma intuitiva de se construir um $\beta$-hom $(\mathcal{C})$-empacotamento. Em particular, o exemplo mostra como construir um 1/4-hom $\left(\mathcal{C}_{2}\right)$-empacotamento em um hipergrafo $\mathcal{H}_{e}$ que possui uma única aresta. Note que a mesma construção é válida ainda que $\mathcal{H}_{e}$ possuísse mais arestas. Neste caso, todas as arestas, a menos da fixada, possuiriam peso 0.

Exemplo 4.11. Seja $\mathcal{H}_{e}$ o hipergrafo 5 -uniforme formado por uma única aresta, i.e, $V\left(\mathcal{H}_{e}\right)=$ $\left\{v_{1}, \ldots, v_{5}\right\}$ e $E\left(\mathcal{H}_{e}\right)=\{e\}=\left\{\left\{v_{1}, \ldots, v_{5}\right\}\right\}$. Seja $V\left(\mathcal{C}_{2}\right)=\left\{x_{1}, x_{2}, x_{3}, x_{4}, x_{5}, x_{6}\right\}$ onde $x_{2}, \ldots, x_{6}$ são os vértices que estão em ambas arestas de $\mathcal{C}_{2}$. Para $1 \leqslant i \leqslant 3$, defina $\varphi_{i}: V\left(\mathcal{C}_{2}\right) \rightarrow V(e)$ tal que $\varphi_{i}\left(x_{1}\right)=\varphi_{i}\left(x_{2}\right)=v_{i}, \varphi_{i}\left(x_{5}\right)=v_{4}, \varphi_{i}\left(x_{6}\right)=v_{5}$ e os vértices restantes em $\mathcal{C}_{2}$ são a pré-imagem dos vértices restantes em $\mathcal{H}_{e}$. Considere $f: \Phi \rightarrow \mathbb{N}$ tal que $f\left(\varphi_{1}\right)=f\left(\varphi_{2}\right)=f\left(\varphi_{3}\right)=1$ e $f(\varphi)=0$ caso contrário. Dessa forma, temos

$$
w_{f}\left(v_{1}\right)=w_{f}\left(v_{2}\right)=w_{f}\left(v_{3}\right)=4 \text { e } w_{f}\left(v_{4}\right)=w_{f}\left(v_{5}\right)=3 .
$$

Note que $f$ não é um $\beta$-hom $\left(\mathcal{C}_{2}\right)$-empacotamento, pois não satisfaz a condição (4.10). Por outro lado, temos que $h=f / 4$ é um (1/4)-hom $\left(\mathcal{C}_{2}\right)$-empacotamento em $\mathcal{H}_{e}$ com

$$
w_{f}\left(v_{1}\right)=w_{f}\left(v_{2}\right)=w_{f}\left(v_{3}\right)=1 \text { e } w_{f}\left(v_{4}\right)=w_{f}\left(v_{5}\right)=\frac{3}{4} .
$$

A Afirmação 4.12 abaixo é uma generalização da ideia utilizada na construção apresentada no Exemplo 4.11 e nos permite uma forma fácil de definir um empacotamento para uma única aresta.

Afirmação 4.12. Sejam $\mathcal{H}$ um hipergrafo $k$-uniforme e $e=\left\{v_{1}, \ldots, v_{k}\right\}$ uma aresta de $\mathcal{H}$. Existe 
um $\frac{1}{2(k-\ell-1)}-\operatorname{hom}\left(\mathcal{C}_{\ell}\right)$-empacotamento $h$ em $\mathcal{H}$ que difere de zero exclusivamente em $e$, tal que $w_{h}\left(v_{i}\right)=1$ para $i \in[k-2]$ e $w_{h}\left(v_{k-1}\right)=w_{h}\left(v_{k}\right)=\frac{k-2}{2(k-\ell-1)}$. Em particular, podemos escalonar o peso de $h$ por qualquer $q \in(0,1]$ e obter um $\frac{q}{2(k-\ell-1)}$-hom $\left(\mathcal{C}_{\ell}\right)$-empacotamento de $\mathcal{H}$ com $w_{h}\left(v_{i}\right)=q$ para $i \in[k-2]$ e $w_{h}\left(v_{k-1}\right)=w_{h}\left(v_{k}\right)=\frac{q(k-2)}{2(k-\ell-1)}$. Mais ainda, temos $w(h)=q(k-2)(1-1 /(k-\ell-1))$.

Demonstração. Sejam $\mathcal{H}$ um hipergrafo $k$-uniforme e $e=\left\{v_{1}, \ldots, v_{k}\right\}$ uma aresta. Considere o conjunto $\mathcal{T}=\left\{I \in[k-2]^{k-2 \ell}: \forall i, j \in[k-2 \ell], I[i]=I[j] \leftrightarrow i=j\right\}$. Para $I \in \mathcal{T}$, seja $\varphi_{I}$ o homomorfismo tal que $\varphi_{I}(i)=\varphi_{I}(2(k-\ell)-i+1)=v_{I[i]}, \varphi_{I}(k)=v_{k}, \varphi_{I}(k-1)=v_{k-1}$ e os vértices restantes de $C_{\ell}$ são a pré-imagem dos vértices restantes de $e$. Seja $\Phi_{\mathcal{T}}=\left\{\varphi_{I}: I \in \mathcal{T}\right\}$. Defina $f: \Phi \rightarrow \mathbb{N}$ tal que para todo $\varphi \in \Phi_{\mathcal{T}}$ temos $f(\varphi)=1$ e para todo $\varphi \notin \Phi_{\mathcal{T}}$ temos $f(\varphi)=0$. Assim, para todo $i \in[k-2]$, temos

$$
\begin{aligned}
w_{f}\left(v_{i}\right) & =\sum_{\substack{u \in V\left(\mathcal{C}_{\ell}\right)\\
}} \sum_{\varphi \in \Phi: v=\varphi(u)} f(\varphi) \\
& =\sum_{\substack{I \in \mathcal{T} ; \\
i \in I}} 2+\sum_{\substack{I \in \mathcal{T} ; \\
i \notin I}} 1 \\
& =2\left(\begin{array}{c}
k-3 \\
k-2 \ell-1
\end{array}\right)(k-2 \ell) !+\left(\begin{array}{c}
k-3 \\
k-2 \ell
\end{array}\right)(k-2 \ell) ! \\
& =\frac{2(k-\ell-1)(k-3) !}{(2 \ell-2) !} .
\end{aligned}
$$

Por outro lado, temos que

$$
\begin{aligned}
w_{f}\left(v_{k-1}\right)=w_{v_{k}} & =\sum_{I \in \mathcal{T}} 1 \\
& =\frac{(k-2) !}{(2 \ell-2) !} .
\end{aligned}
$$

Note que

$$
h=\left(\frac{(2 \ell-2) !}{2(k-\ell-1)(k-3) !}\right) f
$$

é um $\frac{1}{2(k-\ell-1)}-\operatorname{hom}\left(\mathcal{C}_{\ell}\right)$-empacotamento que difere de zero exclusivamente em $e$ com $w_{h}\left(v_{i}\right)=1$ para $i \in[k-2]$ e $w_{h}\left(v_{k-1}\right)=w_{h}\left(v_{k}\right)=\frac{k-2}{2(k-\ell-1)}$.

Dizemos que um hipergrafo $k$-uniforme $\mathcal{R}$ de ordem $t$ é $\beta$-fracionário- $(\ell, \xi)$-extremal se existe uma função $b: V(\mathcal{R}) \rightarrow\{0\} \cup[\beta, 1] \mathrm{com}$

$$
\sum_{v \in V(\mathcal{R})} b(v) \geqslant \frac{2(k-\ell)-1}{2(k-\ell)} t \quad \text { e } \quad \sum_{e \in E(\mathcal{R})} \prod_{v \in e} b(v) \leqslant \xi\left(\begin{array}{l}
t \\
k
\end{array}\right) .
$$

Lembre que um hipergrafo $k$-uniforme $\mathcal{H}=(V, E)$ é chamado de $(\ell, \xi)$-extremal se existe um conjunto $B \subset V$ tal que $|B|=\left\lfloor\frac{2(k-\ell)-1}{2(k-\ell)} n\right\rfloor$ e $e(B) \leqslant \xi\left(\begin{array}{l}n \\ k\end{array}\right)$. Assim, note que, tomando $\beta=1$, a função $b$ pode ser vista como um conjunto de vértices com pesos que representam a mesma ideia do conjunto $B$ na definição de extremalidade.

Na demonstração do Teorema 4.4, o Lema 4.13 abaixo é aplicado no hipergrafo reduzido $\mathcal{R}$ para obter o $\beta$-hom $\left(\mathcal{C}_{\ell}\right)$-empacotamento que utilizamos para obter uma família limitada de $\ell$-caminhos disjuntos que cobre "quase" todos os vértices de $\mathcal{H}$. 
Lema 4.13. Para inteiros $k \geqslant 3$ e $1 \leqslant \ell<k / 2$, existem $C$ e $\gamma_{0}$ tais que, para todos $\alpha>0$ e $\gamma \leqslant \gamma_{0}$, existem $\beta>0$ e $\varepsilon>0$ tais que a seguinte afirmação é válida para todo $t$ suficientemente grande. Seja $\mathcal{R}$ um hipergrafo $k$-uniforme de ordem $t$. Se $R$ não é $\beta$-fracionário- $(\ell, C \gamma)$-extremal e

$$
\operatorname{deg}(K) \geqslant\left(\frac{4(k-\ell)-1}{4(k-\ell)^{2}}-\gamma\right)\left(\begin{array}{l}
t \\
2
\end{array}\right)
$$

para pelo menos $(1-\varepsilon)\left(\begin{array}{c}t \\ k-2\end{array}\right)$ conjuntos $K \in\left(\begin{array}{c}V(\mathcal{R}) \\ k-2\end{array}\right)$ então existe um $\beta$-hom $\left(\mathcal{C}_{\ell}\right)$-empacotamento $h$ com peso pelo menos $(1-\alpha) t$.

Demonstração. Note que é suficiente provar o lema para valores pequenos de $\alpha$. Consequentemente, a quantificação do lema nos permite fixar parâmetros e constantes auxiliares $C^{\prime}$ e $c$ para satisfazer a seguinte hierarquia de constantes

$$
\frac{1}{k}, \frac{1}{\ell} \gg \frac{1}{C^{\prime}} \gg \frac{1}{C} \gg \gamma_{0} \geq \gamma \gg \alpha \gg c, \varepsilon .
$$

Mais ainda, fixamos $\beta$ indutivamente tal que

$$
1=\beta_{0} \gg \beta_{1} \cdots \gg \beta_{\lfloor 1 / c\rfloor}=\beta \quad \text { onde } \quad \frac{\beta_{i}}{\beta_{i+1}} \in \mathbb{N}
$$

e $t \gg 1 / \beta, 1 / c$ suficientemente grande. Em particular, temos que $1 / \beta_{i} \in \mathbb{N}$ para todo $i$. Note que qualquer $\beta_{i}$-hom $\left(\mathcal{C}_{\ell}\right)$-empacotamento é também um $\beta$-hom $\left(\mathcal{C}_{\ell}\right)$-empacotamento, pois $\beta_{i}$ é um múltiplo de $\beta$. Para provar o lema, mostramos que dado um $\beta_{i}$-hom $\left(\mathcal{C}_{\ell}\right)$-empacotamento $h$ com peso $w(h)<(1-\alpha) t$, existe um $\beta_{i+1}$-hom $\left(\mathcal{C}_{\ell}\right)$-empacotamento $h^{\prime}$ com peso $w\left(h^{\prime}\right) \geq w(h)+c t$. Podemos começar com o 1-hom $\left(\mathcal{C}_{\ell}\right)$-empacotamento que possui peso zero e consequentemente, após no máximo $\frac{1}{c}$ passos, obtemos um $\beta$-hom $\left(\mathcal{C}_{\ell}\right)$-empacotamento com peso no mínimo $(1-\alpha) t$.

Para o restante da prova, fixe um $\beta_{i}$-hom $\left(\mathcal{C}_{\ell}\right)$-empacotamento $h$ com peso $w(h)<(1-\alpha) t$ e assuma, por contradição, que não existe um $\beta_{i+1}$-hom $\left(\mathcal{C}_{\ell}\right)$-empacotamento com peso pelo menos $w(h)+c t$. Portanto, por (4.11), existem pelo menos $\alpha t / 2$ vértices $v \in V(\mathcal{R}) \operatorname{com} w_{h}(v)<1-\alpha / 2$ e podemos fixar um conjunto $W$ de vértices com $|W|=\alpha t / 2$.

Para melhorar $h$, utilizamos apenas arestas que intersectam $W$. Dessa forma, podemos assumir que

$$
(1-2 \alpha) t \leq w(h)<(1-\alpha) t
$$

pois, caso contrário, poderíamos adicionar arestas em $V \backslash W$ até que (4.15) seja trivialmente atingindo e removemos essas arestas após melhorar o peso de $h$.

Para o restante da prova, assumimos que $\Phi$ é um multiconjunto no qual incluímos $\varphi \in \Phi$ com multiplicidade $\frac{h(\varphi)}{\beta_{i}}$. Dessa forma, podemos assumir que $\Phi$ é composto apenas por homomorfismos com pesos positivos e que $h: \Phi \rightarrow\left\{\beta_{i}\right\}$. Considerando $\Phi$ e $h$ como acima e por (4.11), obtemos os seguintes limitantes para o tamanho $\Phi$ :

$$
(1-2 \alpha) \frac{t}{\beta_{i} v\left(\mathcal{C}_{\ell}\right)} \leq|\Phi|<(1-\alpha) \frac{t}{\beta_{i} v\left(\mathcal{C}_{\ell}\right)}<\frac{t}{\beta_{i}} .
$$

Também identificamos um homomorfismo $\varphi \in \Phi$ pelos, não necessariamente distintos, vértices $\left(v_{1}, \ldots, v_{2 k-2 \ell}\right)$ da sua imagem, onde $\left\{v_{1}, \ldots, v_{k}\right\}$ e $\left\{v_{2 k-2 \ell+1}, \ldots, v_{2 k-2 \ell}\right\}$ formam arestas em $\mathcal{R}$. Nos referimos aos elementos de $\Phi$ como cerejas. 
Considere os $(k-2)$-conjuntos em $W$ que satisfazem a condição de grau mínimo do lema. Dessa forma, como $\varepsilon \ll \alpha$ (ver (4.14)), podemos encontrar, utilizando uma abordagem gulosa, uma coleção $\mathcal{W}$ na qual os elementos são dois a dois disjuntos e cobrem pelo menos $\frac{1}{2}|W|$ vértices. Note que

$$
|\mathcal{W}| \geq \frac{|W|}{2(k-\ell)}>\frac{\alpha t}{4 k}
$$

Para $K \in \mathcal{W}$, consideramos o grafo link $L_{K}$ de $K$ em $\mathcal{R}$. Encontraremos pares de cerejas $\mathcal{C}, \mathcal{C}^{\prime} \in \Phi$ nos quais o grafo link $L_{k}\left(\mathcal{C}, \mathcal{C}^{\prime}\right)$ nos permite encontrar um empacotamento com o peso maior. Para isso, consideramos somente as arestas em $L_{K}\left(\mathcal{C}, \mathcal{C}^{\prime}\right)$ que contêm um vértice em $\mathcal{C}$ e um vértice em $\mathcal{C}^{\prime}$. No máximo $\frac{t}{\beta_{i}}\left(\begin{array}{c}v\left(\mathcal{C}_{\ell}\right) \\ 2\end{array}\right) \leqslant \gamma\left(\begin{array}{l}t \\ 2\end{array}\right)$ arestas possuem as ambas extremidades no mesmo $\mathcal{C}$ e no máximo $\frac{\alpha t^{2}}{2} \leq \gamma\left(\begin{array}{l}t \\ 2\end{array}\right)$ arestas contêm um vértice de $W$. Seja $L_{K}^{\prime}$ o grafo obtido de $L_{K}$ após remover todas essas arestas. Por (4.13), sabemos que

$$
e\left(L_{K}^{\prime}\right) \geq\left(\frac{4(k-\ell)-1}{4(k-\ell)^{2}}-3 \gamma\right)\left(\begin{array}{l}
t \\
2
\end{array}\right)
$$

Dizemos que $\mathcal{C}, \mathcal{C}^{\prime} \in \Phi$ é um par extremal para $K$ se existem $u_{\mathcal{C}} \in \mathcal{C}$ e $u_{\mathcal{C}^{\prime}} \in \mathcal{C}^{\prime}$ tais que $L_{K}^{\prime}\left(\mathcal{C}, \mathcal{C}^{\prime}\right)$ contêm todas as arestas incidentes a esses dois vértices. Em particular, dizemos que $u_{\mathcal{C}}$ e $u_{\mathcal{C}^{\prime}}$ são vértices especiais para $\mathcal{C}$ e $\mathcal{C}^{\prime}$. Mostramos a seguir que, para a maioria dos conjuntos $K \in \mathcal{W}$, o grafo link bipartido entre a maioria dos pares $\mathcal{C}$ e $\mathcal{C}^{\prime}$ são extremais para $K$.

Afirmação 4.14. Existe um elemento $u_{\mathcal{C}} \in \mathcal{C}$ para cada $\mathcal{C} \in \Phi$ tal que a seguinte afirmação é verdadeira. Para pelo menos $(1-\gamma)|\mathcal{W}|$ conjuntos $K \in \mathcal{W}$ pelo menos uma fração $\left(1-C^{\prime} \gamma\right)$ de pares de cerejas $\mathcal{C}, \mathcal{C}^{\prime} \in \Phi$ são extremais para $K$ com vértices especiais $u_{\mathcal{C}}$ e $u_{\mathcal{C}^{\prime}}$.

Demonstração. A prova da afirmação consiste em três passos. Primeiro, mostramos que se $L_{K}\left(\mathcal{C}, \mathcal{C}^{\prime}\right)$ contêm mais que $4(k-\ell)-1$ arestas então é possível fazer uma "melhoria local" em $w(h)$ de pelo menos $\beta_{i} / 4$. No segundo passo, limitamos a quantidade de melhorias locais que podemos efetuar, pois caso contrário podemos combinar todas as melhorias locais e obter um $h^{\prime}$ com o peso $w\left(h^{\prime}\right) \geq w(h)+c t$ e dessa forma concluiríamos a prova do Lema 4.13. No último passo, utilizamos o limitante obtido no segundo passo para mostrar que um $L_{K}\left(\mathcal{C}, \mathcal{C}^{\prime}\right)$ "típico" contêm somente $4(k-\ell)-1$ arestas e possui as propriedades desejadas.

Para o primeiro passo, consideramos dois casos. No primeiro caso, suponha que existe um emparelhamento com três arestas em $L_{K}^{\prime}\left(\mathcal{C}, \mathcal{C}^{\prime}\right)$. Aplicamos a Afirmação 4.12 nas arestas de $\mathcal{R}$ relacionadas às arestas do emparelhamento com $q=\beta_{i} / 3, v_{k-1}$ e $v_{k}$ como sendo os vértices de $\mathcal{C}$ e $\mathcal{C}^{\prime}$ e reduzimos os pesos das cerejas $\mathcal{C}$ e $\mathcal{C}^{\prime}$ para $h^{\prime}(\mathcal{C})=h^{\prime}\left(\mathcal{C}^{\prime}\right)=\left(1-\frac{k-2}{6(k-\ell-1)}\right) \beta_{i}$. Note que isso é possível, pois como os vértices de $K$ possuem peso no máximo $1-\alpha / 2$ e $h$ leva homomorfismos em múltiplos de $\beta_{i}$ então $w_{h}(v) \leq 1-\beta_{i}$ para todo $v \in K$. Dessa forma, considerando os vértices nos quais o peso mudou, é fácil ver

$$
w\left(h^{\prime}\right)=w(h)+\left(k-2-(4 k-4 \ell-6) \cdot \frac{k-2}{6(k-\ell-1)}\right) \beta_{i} \geqslant w(h)+\frac{1}{3} \beta_{i} .
$$

Assim, obtemos uma melhoria de pelo menos $\beta_{i} / 3$ ao peso de $h$.

Para o segundo caso, suponha que não existe um emparelhamento de tamanho três. Note que para $\mathcal{C}, \mathcal{C}^{\prime} \in \Phi$, o grafo $L_{K}^{\prime}\left(\mathcal{C}, \mathcal{C}^{\prime}\right)$ é um grafo bipartido com classes de tamanho $2(k-\ell)$. Pelo 
Teorema de König (Teorema 2.9), o emparelhamento máximo em um grafo bipartido possui o mesmo tamanho que a menor cobertura de vértices. Se não existe emparelhamento de tamanho três então existe uma cobertura de tamanho dois e consequentemente existem no máximo $4(k-\ell)$ arestas em $L_{K}^{\prime}\left(\mathcal{C}, \mathcal{C}^{\prime}\right)$. Dessa forma, a única configuração que permite existir exatamente $4(k-\ell)$ arestas em $L_{K}^{\prime}\left(\mathcal{C}, \mathcal{C}^{\prime}\right)$ e nenhum emparelhamento de tamanho três é se os dois vértices da cobertura estão na mesma classe, digamos em $\mathcal{C}$, e suas vizinhanças estão todas em $\mathcal{C}^{\prime}$. Em particular, existem duas arestas para cada um dos dois vértices da cobertura usando quatro vértices distintos em $\mathcal{C}^{\prime}$. Aplicando a Afirmação 4.12 nas arestas do hipergrafo $\mathcal{R}$ correspondentes a essas quatro arestas, com $q=\beta_{i} / 4, v_{k-1}$ e $v_{k}$ como sendo os vértices em $\mathcal{C}$ e $\mathcal{C}^{\prime}$ e reduzindo os pesos das cerejas para $h^{\prime}(\mathcal{C})=\left(1-\frac{k-2}{4(k-\ell-1)}\right) \beta_{i}$ e $h^{\prime}\left(\mathcal{C}^{\prime}\right)=\left(1-\frac{k-2}{8(k-\ell-1)}\right) \beta_{i}$ obtemos $h^{\prime}$ com

$$
\begin{aligned}
w\left(h^{\prime}\right) & =w(h)+\left(k-2-(2 k-2 \ell-2) \cdot \frac{k-2}{4(k-\ell-1)}-(2 k-2 \ell-4) \cdot \frac{k-2}{8(k-\ell-1)}\right) \beta_{i} \\
& \geqslant w(h)+\frac{k-2}{4} \beta_{i} \geqslant w(h)+\frac{1}{4} \beta_{i} .
\end{aligned}
$$

Isso estabelece a melhoria local para este caso e conclui o primeiro passo.

Para o segundo passo, suponha que existem pelo menos $\gamma|\mathcal{W}| / 2$ conjuntos $K \in \mathcal{W}$ tais que para pelo menos $\gamma|\Phi|^{2}$ pares de cerejas essas melhorias são possíveis. Utilizando uma abordagem gulosa, podemos aplicar essas melhorias locais utilizando cada cereja de $\mathcal{C} \in \Phi$ no máximo uma vez, para todo $K$, para aumentar o peso de $h$ o máximo possível. Este procedimento acaba ou quando todo $K \in \mathcal{W}$ possui um vértice que já foi saturado e, neste caso, aumentamos o peso total em pelo menos $\frac{\alpha}{2} \gamma|\mathcal{W}| / 2$, ou quando, para todo $K \in \mathcal{W}$, para cada um dos $\gamma|\Phi|^{2}$ pares de cerejas, pelo menos uma foi utilizada. Como qualquer coleção de $\gamma|\Phi|^{2}$ pares de cerejas contêm pelo menos $\gamma|\Phi| / 4$ pares dois a dois disjuntos, então o último caso implica que aplicamos pelo menos $\gamma|\Phi| / 8$ melhorias locais anteriormente.

Em resumo, mostramos que se para pelos menos $\gamma|\mathcal{W}| / 2 \geqslant \gamma \frac{\alpha t}{8 k}$ (ver (4.17)) conjuntos $K \in \mathcal{W}$ for possível aplicar a melhoria local para pelo menos $\gamma|\Phi|^{2}$ pares de cerejas, então nós podemos adicionar essas melhorias locais a $h$ obtendo um $\beta_{i+1}$-hom $\left(\mathcal{C}_{\ell}\right)$-empacotamento $h^{\prime \prime}$ com peso pelo menos

$$
w\left(h^{\prime \prime}\right) \geq w(h)+\min \left\{\frac{\alpha}{2}, \gamma \frac{\alpha t}{8 k}, \frac{\beta_{i}}{4}, \frac{\gamma}{8}|\Phi|\right\} \geq w(h)+c t,
$$

e assim a prova do Lema 4.13 estaria concluída.

Consequentemente, para o terceiro passo, podemos considerar somente os conjuntos $K \in \mathcal{W}$ para os quais no máximo uma fração $\gamma$ de pares de cerejas $\mathcal{C}$ e $\mathcal{C}^{\prime}$ induzem no mínimo $4(k-\ell)$ arestas ou um emparelhamento de tamanho três. Utilizando um argumento de média e (4.18), temos que a quantidade média de arestas entre duas cerejas é pelo menos $4(k-\ell)-1-12 \gamma(k-\ell)^{2}$. Portanto, como $C^{\prime}$ foi escolhido grande o suficiente, no máximo $\left(C^{\prime}-1\right) \gamma$ pares de cerejas não possuem exatamente $4(k-\ell)-1$ arestas.

Novamente pelo Teorema de König (Teorema 2.9), o único caso em que podemos ter $4(k-\ell)-1$ arestas e nenhum emparelhamento de tamanho três é se existir um vértice em cada lado que é adjacente a todos os vértices do lado oposto. Assuma que esses vértices em $\mathcal{C}$, digamos $u$ e $v$, são diferentes para $L_{K}^{\prime}\left(\mathcal{C}, \mathcal{C}^{\prime}\right)$ e $L_{K^{\prime}}^{\prime}\left(\mathcal{C}, \mathcal{C}^{\prime \prime}\right)$. Neste caso, podemos configurar os pesos da seguinte forma. Escolha quatro arestas incidentes em $u$ em $L_{K}^{\prime}\left(\mathcal{C}, \mathcal{C}^{\prime}\right)$ e quatro para $v$ em $L_{K^{\prime}}^{\prime}\left(\mathcal{C}, \mathcal{C}^{\prime \prime}\right)$. Aplicamos a Afirmação 4.12 nessas oito arestas com $q=\beta_{i} / 8, v_{k}$ e $v_{k-1}$ como sendo os vértices em $\mathcal{C}$ e $\mathcal{C}^{\prime}$ e 
reduzimos os pesos das cerejas para $h^{\prime}(\mathcal{C})=\left(1-\frac{k-2}{4(k-\ell-1)}\right) \beta_{i}$ e $h^{\prime}\left(\mathcal{C}^{\prime}\right)=h^{\prime}\left(\mathcal{C}^{\prime \prime}\right)=\left(1-\frac{k-2}{16(k-\ell-1)}\right) \beta_{i}$. Da mesma forma que foi feita no primeiro passo, isso nos fornece um melhoria local de $\frac{1}{4} \beta_{i}$.

Se para pelo menos $\gamma|\mathcal{W}| / 2$ conjuntos $K \in \mathcal{W}$ podemos definir essa melhoria local para pelo menos $\gamma|\Phi|^{2}$ pares extremais, de forma similar ao segundo passo, podemos concluir a prova do Lema 4.13. Portanto, para cada cereja $\mathcal{C}$ podemos fixar um vértice $u_{\mathcal{C}}$ tal que para pelo menos $(1-\gamma)|\mathcal{W}|$ conjuntos $K \in \mathcal{W}$ pelo menos uma fração $\left(1-C^{\prime} \gamma\right)$ de pares de cerejas $\mathcal{C}, \mathcal{C}^{\prime} \in \Phi$ são extremais para $K$ com vértices especiais $u_{\mathcal{C}}$ e $u_{\mathcal{C}^{\prime}}$.

Dizemos que $\mathcal{C} \in \Phi$ é boa se está contida em pelo menos $\frac{1}{2}|\Phi|$ pares extremais e ruim caso contrário. Dessa forma, pela Afirmação 4.14, no máximo $5 C^{\prime} \gamma|\Phi|$ cerejas são ruins. Mais ainda, para todo $v \in V$, denotamos por $\Phi_{\mathrm{RUIM}}(v)$ a quantidade de cerejas ruins $C \in \Phi$ que contêm o vértice $v$.

Para completar a prova, mostramos que podemos encontrar um emparelhamento $M$ tal que todo vértice $v \in e \in M$ está contido em "muitas" cerejas boas. Para cada cereja boa $\mathcal{C} \in \Phi$ existem muitas escolhas para $\mathcal{C}^{\prime}$ e $K \in \mathcal{W}$ tais que $\mathcal{C}$ e $\mathcal{C}^{\prime}$ são extremais em relação a $K$. Assim, podemos redistribuir os pesos de forma a transferir pesos dos vértices não especiais de $\mathcal{C}$ e $\mathcal{C}^{\prime}$ para $K$, o que irá reduzir o peso de $v$ (garantimos que $v$ é um vértice não especial). Repetindo esse processo para todo $v \in e$, isso nos permitirá obter uma melhoria local para o empacotamento e repetindo esse processo para várias arestas $e \in M$, obtemos a melhoria local desejada.

Definimos a função $a: v(\mathcal{R}) \rightarrow[0,1]$ por $v \rightarrow \beta_{i} \sum_{\mathcal{C} \in \Phi} \mathbb{1}_{v}\left(u_{\mathcal{C}}\right)$. Note que $a$ representa o quanto um vértice foi utilizado como vértice especial. Como toda cereja contêm $2(k-\ell)$ vértices, por (4.16), temos que $\sum_{v \in V(\mathcal{R})} a(v) \leq \frac{t}{2(k-\ell)}$ e portanto, podemos utilizar a não $\beta$-fracionário- $(l, \gamma)$-extremalidade de $\mathcal{R}$ para os pesos $b(\cdot)=1-a(\cdot)$ e obtemos

$$
\sum_{e \in E(\mathcal{R})} \prod_{v \in e} b(v) \geq C \gamma\left(\begin{array}{l}
t \\
k
\end{array}\right) .
$$

Como existem no máximo $5 C^{\prime} \gamma|\Phi|$ cerejas ruins, então elas contribuem com no máximo

$$
\beta_{i} \sum_{v \in V(\mathcal{R})}\left|\Phi_{\mathrm{RUIM}}(v)\right| \leq \beta_{i} v\left(\mathcal{C}_{\ell}\right) 5 C^{\prime} \gamma|\Phi| \leq 5 C^{\prime} \gamma t
$$

Queremos utilizar somente cerejas boas para redistribuir os pesos. Portanto, considere a função $b^{\prime}: V(\mathcal{R}) \rightarrow[0,1]$ dada por

$$
b^{\prime}(v)=\max \left\{0, b(v)-\beta_{i}\left|\Phi_{\mathrm{RUIM}}(v)\right|\right\}
$$

e, por (4.19), (4.16) e $C^{\prime} \ll C$, temos

$$
\sum_{e \in E(\mathcal{R})} \prod_{v \in e} b^{\prime}(v) \geqslant \frac{C}{2} \gamma\left(\begin{array}{l}
t \\
k
\end{array}\right) .
$$

Por um argumento de dupla contagem, obtemos que existe um emparelhamento $M \subset E(\mathcal{R})$ com

$$
\sum_{e \in M} \prod_{v \in e} b^{\prime}(v) \geq \frac{C}{2} \gamma \frac{t}{k}
$$


e como $b^{\prime}(v) \in[0,1]$, temos

$$
\sum_{e \in M} k \min _{v \in e}\left\{b^{\prime}(v)\right\} \geq \sum_{e \in M} k \prod_{v \in e} b^{\prime}(v) \geq \frac{C}{2} \gamma t
$$

Em particular, podemos assumir que $\min _{v \in e}\left\{b^{\prime}(v)\right\}>0$ para todo $e \in M$. Mais ainda, da definição da função $b^{\prime}(c)$ e da definição de $\beta_{i}$ segue que $\min _{v \in e}\left\{b^{\prime}(v)\right\} \geqslant \beta_{i}$ para todo $e \in M$.

Para cada vértice $v \in V(M)$, consideramos as cerejas boas que contêm o vértice $v$ como vértice não especial. Assuma que temos $K \in \mathcal{W}$ e um par extremal $\mathcal{C}$ e $\mathcal{C}^{\prime}$ tais que $v$ não é um vértice especial em $\mathcal{C}$. O grafo link $L_{K}\left(\mathcal{C}, \mathcal{C}^{\prime}\right)$ contêm todas as arestas incidentes aos dois vértices especiais. Assim, definimos um deslocamento de peso local da seguinte forma: coloque pesos $\frac{1}{2(k-\ell)-1} \cdot \frac{k-2}{4(k-\ell-1)} \beta_{i}$ para os vértices de todas as arestas incidentes a exatamente um dos vértices especiais, $\beta_{i}$ para os vértices de $K$ e coloque $h^{\prime}(\mathcal{C})=h^{\prime}\left(\mathcal{C}^{\prime}\right)-\left(1-\frac{k-2}{4(k-\ell)-2}\right) \beta_{i}$. De forma similar aos cálculos realizados no começo da demonstração desse lema, obtemos um $\beta_{i+1}$-hom-empacotamento $h^{\prime} \operatorname{com} w\left(h^{\prime}\right)=w(h)$. Por outro lado, o peso do vértice $v$ e de todos os outros vértices não especiais em $L_{k}\left(\mathcal{C}, \mathcal{C}^{\prime}\right)$ são reduzidos por $\frac{k-2}{4(k-\ell)-2} \beta_{i}$, i.e.

$$
w_{h^{\prime}}(v)=w_{h}(v)-\frac{k-2}{4(k-\ell)-2} \beta_{i} .
$$

Dessa forma, pela definição de $b^{\prime}(v)$, temos que existem pelo menos $b^{\prime}(v) / \beta_{i}$ cerejas boas que contêm $v$ como vértice não especial e podemos aplicar o deslocamento de peso local no máximo $\min _{u \in e}\left\{b^{\prime}(u)\right\} / \beta_{i}$ vezes para um vértice $v \in e \in M$.

Para toda aresta $e \in M$, gostaríamos de aplicar esses deslocamentos de pesos locais para todo vértice $v \in e$, onde circulamos através de todos os $k$ vértices e aplicamos o deslocamento a cada um deles. Em outras palavras, eventualmente reduzimos os pesos de todos os vértices de $e$. Note que podemos aplicar esses deslocamentos de pesos locais usando $K, \mathcal{C}$ e $\mathcal{C}^{\prime}$ a menos que já tenhamos saturados os vértices em $K$ ou usado uma das cerejas anteriores. O procedimento para assim que atingirmos um vértice para o qual não é possível realizar o deslocamento de pesos locais.

Primeiro discutimos o caso ideal no qual este procedimento não para, i.e., para todo $e \in M \mathrm{e}$ para todo $v \in e$ aplicamos $\min _{u \in e}\left\{b^{\prime}(u)\right\} / \beta_{i}$ deslocamentos locais de pesos. Neste caso, para todo $e \in M$, podemos reduzir o peso de todos os vértices $v \in e$ para no mínimo

$$
\frac{1}{\beta_{i}} \min _{u \in e}\left\{b^{\prime}(u)\right\} \frac{k-2}{4(k-\ell)-2} \beta_{i}=\frac{k-2}{4(k-\ell)-2} \min _{u \in e}\left\{b^{\prime}(u)\right\} .
$$

Consequentemente, aplicando a Afirmação 4.12, incrementamos o empacotamento na aresta $e$ pelo mesmo valor. Repetindo este processo para todo $e \in M$, obtemos um $\beta_{i+1}$-hom-empacotamento $h^{\prime \prime}$ satisfazendo

$$
w\left(h^{\prime \prime}\right) \geq w(h)+\sum_{e \in M} k \frac{k-2}{4(k-\ell)-2} \min _{u \in e}\left\{b^{\prime}(u)\right\} \stackrel{(4.21)}{\geq} w(u)+\frac{C \gamma t}{2} \frac{k-2}{4(k-\ell)-2} \stackrel{(4.14)}{\geq} w(h)+c t,
$$

o que conclui a prova desse caso.

No caso no qual o procedimento para, existe algum $v \in e \in M$ e uma cereja boa $\mathcal{C}$ para $v$ tal que $\mathcal{C}$ não pode ser utilizada para o deslocamento local de pesos. Isso significa que, como $\mathcal{C}$ é uma cereja boa, existem pelo menos $|\mathcal{W}| / 2$ conjuntos $K \in \mathcal{W}$ que possuem um vértice saturado ou existem 
pelo menos $|\Phi| / 2$ cerejas que já foram utilizadas em deslocamentos locais de pesos anteriormente. No caso em que existem $|\mathcal{W}| / 2$ conjuntos $K \in \mathcal{W}$ que contêm vértices saturados, então cada um desses vértices foram usados em pelo menos $\frac{\alpha}{2 \beta_{i}}$ deslocamentos locais de pesos. Portanto, aplicamos no total

$$
\frac{|\mathcal{W}|}{2} \frac{\alpha}{2 \beta_{i}} \stackrel{(4.17)}{\geq} \frac{\alpha t}{8 k} \frac{\alpha}{2 \beta_{i}}
$$

deslocamentos de pesos. Por outro lado, se todas as $|\Phi| / 2$ cerejas possíveis, $\mathcal{C}^{\prime}$, foram utilizadas anteriormente em deslocamentos locais de pesos então aplicamos pelo menos $|\Phi| / 4$ deslocamentos locais de pesos. Como no caso ideal, usando a Afirmação 4.12, concluímos que aumentamos o empacotamento nas arestas de $M$ e obtemos um $\beta_{i+1}$-hom-empacotamento $h^{\prime \prime}$ com

$$
w\left(h^{\prime \prime}\right) \geq w(h)+\left(\min \left\{\frac{\alpha^{2} t}{16 k \beta_{i}}, \frac{|\Phi|}{4}\right\}-k\right) \frac{(k-2) \beta_{i}}{4(k-\ell)-2} \stackrel{(4.14),(4.16)}{\geq} w(h)+c t
$$

o que conclui a prova do Lema 4.13.

Agora, desejamos transferir o $\beta$-hom $\left(\mathcal{C}_{\ell}\right)$-empacotamento de $\mathcal{R}$ para um empacotamento em caminhos de $\mathcal{H}$. Para isso, vamos usar o seguinte resultado.

Lema 4.15 (Lemma 2.7 [10]). Dados $k \geqslant 3,1 \leqslant \ell<k / 2$ inteiros e $\varepsilon, d>0$ tais que $d>2 \varepsilon$. Seja $m>\frac{k^{2}}{\varepsilon^{2}(d-\varepsilon)}$. Suponha que $\mathcal{V}=\left(V_{1}, \ldots, V_{k}\right)$ é uma $k$-upla $(\varepsilon, d)$-regular com

$$
\left|V_{1}\right|=\cdots=\left|V_{2 \ell}\right|=m \text { e }\left|V_{2 \ell+1}\right|=\cdots=\left|V_{k}\right|=2 m \text {. }
$$

Então existem no máximo $\frac{2 k}{(d-\varepsilon) \varepsilon} \ell$-caminhos disjuntos que cobrem pelo menos $(1-2 k \varepsilon) m$ vértices de $\mathcal{V}$.

Finalmente, usando o Lema 4.15 nas arestas do $\beta$-hom $\left(\mathcal{C}_{\ell}\right)$-empacotamento de $\mathcal{R}$ dado pelo Lema 4.13, obtemos um empacotamento em caminhos de $\mathcal{H}$ do tamanho desejado.

Lema 4.16 (Lema de Empacotamento em Caminhos). Para todos os inteiros $k \geqslant 3$ e $1 \leqslant \ell<k / 2$, existem $C, \gamma_{0}>0$, tais que, para todo $\alpha>0, \gamma<\gamma_{0}$, existe um inteiro $s$ de forma que o seguinte vale para todo $n$ suficientemente grande. Seja $\mathcal{H}$ um hipergrafo $k$-uniforme de ordem $n$ e

$$
\delta_{k-2}(\mathcal{H}) \geqslant\left(\frac{4(k-\ell)-1}{4(k-\ell)^{2}}-\gamma\right)\left(\begin{array}{l}
n \\
2
\end{array}\right)
$$

Então ou existe uma família $\ell$-caminhos disjuntos com no máximo $s$ elementos que cobre pelo menos $(1-\alpha) n$ vértices de $\mathcal{H}$ ou $\mathcal{H}$ é $(\ell, C \gamma)$-extremal.

Demonstração. Sejam $k \geqslant 3$ e $1 \leqslant \ell<k / 2$ dados e sejam $C^{\prime}$ e $\gamma_{0}^{\prime}$ as constantes dadas pelo Lema 4.13 para $k$ e $\ell$. Defina $C=6 C^{\prime}$ e $\gamma_{0}=\frac{\gamma_{0}^{\prime}}{4}$, e seja $\alpha>0$ e $\gamma<\gamma_{0}$. Seguindo a quantificação do Lema 4.13 com $\frac{\alpha}{2}$ e $\gamma$, obtemos $\beta$, $\varepsilon^{\prime}$ e um $t_{0}$ suficientemente grande. Seja $\varepsilon$ suficientemente pequeno. Então o Lema da Regularidade Fraca (Lema 2.10) para $\varepsilon_{0}=\frac{\beta \varepsilon}{2}$ e $t_{0}$ garante um $T_{0}$. Sejam $s$ uma constante suficientemente grande e $\mathcal{H}$ um hipergrafo $k$-uniforme de ordem $n$ suficientemente grande tal que

$$
\delta_{k-2}(\mathcal{H}) \geqslant\left(\frac{4(k-\ell)-1}{4(k-\ell)^{2}}-\gamma\right)\left(\begin{array}{l}
n \\
2
\end{array}\right)
$$

Pelo Lema da Regularidade (Lema 2.10), existe uma partição $\varepsilon_{0}$-regular $V_{0} \cup \cdots \cup V_{t}$ de $\mathcal{H}$ com $\left|V_{1}\right|=\cdots=\left|V_{t}\right|=m,\left|V_{0}\right| \leq \varepsilon_{0} n$ e $t_{0} \leqslant t \leqslant T_{0}$ e um hipergrafo reduzido $\mathcal{R}=\mathcal{R}\left(\varepsilon_{0}, \gamma\right)$ de ordem $t$ 
que, pelo Lema 2.11, não satisfaz

$$
\operatorname{deg}_{\mathcal{R}}(K) \geqslant\left(\frac{4(k-\ell)-1}{4(k-\ell)^{2}}-4 \gamma\right)\left(\begin{array}{l}
t \\
2
\end{array}\right)
$$

para pelo menos $\left(1-\sqrt{\varepsilon_{0}}\right)\left(\begin{array}{c}t \\ k-2\end{array}\right) \geq\left(1-\varepsilon^{\prime}\right)\left(\begin{array}{c}t \\ k-2\end{array}\right)$ conjuntos $K \in\left(\begin{array}{c}{[t]} \\ k-2\end{array}\right)$. Dividimos o restante da prova em dois casos, dependendo se $\mathcal{R}$ é $\beta$-fracionário- $\left(\ell, 4 C^{\prime} \gamma\right)$-extremal ou não.

Suponha que $\mathcal{R}$ não é $\beta$-fracionário- $\left(\ell, 4 C^{\prime} \gamma\right)$-extremal. Então o Lema 4.13 implica que existe um $\beta$-hom $\left(\mathcal{C}_{\ell}\right)$-empacotamento $h$ de $\mathcal{R}$ com peso $\left(1-\frac{\alpha}{2}\right)$ t. Seja $\Phi^{+}$o conjunto de homomorfismos de $\mathcal{C}_{\ell}$ em $\mathcal{R} \operatorname{com} h(\varphi)>0$, em particular temos $h(\varphi) \geqslant \beta$. Utilizamos o Lema 4.15 para obter $\ell$-caminhos cobrindo quase todos os vértices de $\mathcal{H}$. Para isso, dividimos as classes da partição de acordo com o empacotamento $h$. Seja $\left\{R_{1}^{\varphi}, \ldots, R_{2 k-2 \ell}^{\varphi}\right\}_{\varphi \in \Phi^{+}}$uma família tal que para todo $\varphi \neq \varphi^{\prime} \in \Phi^{+}$temos

- $R_{i}^{\varphi} \subset V_{\varphi(i)}$ para todo $i \in[2 k-2 \ell]$,

- $R_{i}^{\varphi} \cap R_{j}^{\varphi^{\prime}}=\emptyset$ para todo $i, j \in[2 k-2 \ell]$,

- $\left|R_{i}^{\varphi}\right|=2\left\lfloor\frac{h(\varphi) m}{2}\right\rfloor$ para todo $i \in[2 k-2 \ell]$.

Para cada $\varphi \in \Phi^{+}$e todo $i \in\{k-2 \ell+1, \ldots, k\}$, seja $S_{i}^{\varphi} \cup U_{i}^{\varphi}=R_{i}^{\varphi}$ uma partição de $R_{i}^{\varphi}$ em duas classes de mesmo tamanho. Note que

$$
\left(R_{1}^{\varphi}, \ldots, R_{k-2 \ell}^{\varphi}, S_{k-2 \ell+1}^{\varphi}, \ldots, S_{k}^{\varphi}\right) \quad \text { e }\left(U_{k-2 \ell+1}^{\varphi}, \ldots, U_{k}^{\varphi}, R_{k+1}^{\varphi}, \ldots, R_{2 k-2 \ell}^{\varphi}\right)
$$

são $(\varepsilon, \gamma)$-regulares. Então, pelo Lema 4.15, para ambos os pares acima existem no máximo $\frac{2 k}{(\gamma-\varepsilon) \varepsilon}$ $\ell$-caminhos que cobrem pelo menos $(1-2 k \varepsilon)\left|R_{i}^{\varphi}\right|$ vértices. Aplicando isso para todos os homomorfismos $\varphi \in \Phi^{+}$, e como $\left|\Phi^{+}\right|$independe de $n$ e $s$ é suficientemente grande, obtemos no máximo $s$ $\ell$-caminhos.

Afirmamos que a quantidade de vértices em $V(\mathcal{H})$ que não são cobertos por esses caminhos é no máximo $\alpha n$. Para isso, note que os vértices não são cobertos por esses caminhos são os vértices da classe $V_{0}$, os vértices que não estão contidos em nenhum $R_{i}^{\varphi}$ e aqueles que estão contidos em algum $R_{i}^{\varphi}$ e não está contido em nenhum $\ell$-caminho. Devido ao peso do $\beta$-hom $\left(C_{\ell}\right)$-empacotamento, no máximo $\frac{\alpha}{2} n$ vértices não estão em nenhum $R_{i}^{\varphi}$ e perdemos no máximo $\frac{2 t}{\beta}$ vértices por causa dos arredondamentos na definição dos conjuntos $R_{i}^{\varphi}$. Os $\ell$-caminhos cobrem todos a menos de uma fração $k \varepsilon$ de vértices em $\bigcup_{i, \varphi} R_{i}^{\varphi}$. Consequentemente, a quantidade total de vértices que não são cobertos é no máximo

$$
\varepsilon_{0} n+\frac{\alpha}{2} n+\frac{2 t}{\beta}+k \varepsilon n<\alpha n
$$

Agora suponha que $\mathcal{R}$ é $\beta$-fracionário- $\left(\ell, 4 C^{\prime} \gamma\right)$-extremal. Pela definição de $\beta$-fracionário- $\left(\ell, 4 C^{\prime} \gamma\right)$ extremal, existe uma função $b: V(\mathcal{R}) \rightarrow\{0\} \cup[\beta, 1]$ com

$$
\sum_{v \in V(\mathcal{R})} b(v) \geqslant \frac{2(k-\ell)-1}{2(k-\ell)} t \text { e } \sum_{e \in E(\mathcal{R})} \prod_{v \in e} b(v) \leqslant 4 C^{\prime} \gamma\left(\begin{array}{l}
t \\
k
\end{array}\right) .
$$

Para cada $i \in[t]$, fixamos um subconjunto $A_{i} \subseteq V_{i} \operatorname{com}\left|A_{i}\right|=\left\lfloor b(i)\left|V_{i}\right|\right\rfloor$ e definimos $B=\bigcup_{i \in[t]} A_{i}$. 
Portanto, pela definição do hipergrafo reduzido $\mathcal{R}=\mathcal{R}\left(\varepsilon_{0}, \gamma\right)$, temos

$$
\begin{aligned}
e_{\mathcal{H}}(B) & \leqslant \sum_{e \in E(\mathcal{R})} \prod_{v \in e}\left(b(v) \frac{n}{t}\right)+\left(\begin{array}{l}
t \\
k
\end{array}\right) \gamma\left(\frac{n}{t}\right)^{k}+\varepsilon_{0}\left(\begin{array}{l}
t \\
k
\end{array}\right)\left(\frac{n}{t}\right)^{k}+t\left(\begin{array}{c}
n / t \\
2
\end{array}\right)\left(\begin{array}{c}
n \\
k-2
\end{array}\right) \\
& \leqslant 4 C^{\prime} \gamma\left(\begin{array}{l}
n \\
k
\end{array}\right)+\gamma\left(\begin{array}{l}
n \\
k
\end{array}\right)+\varepsilon_{0}\left(\begin{array}{l}
n \\
k
\end{array}\right)+\frac{k(k-1)}{2 t}\left(\begin{array}{l}
n \\
k
\end{array}\right) \\
& \leqslant 5 C^{\prime} \gamma\left(\begin{array}{l}
n \\
k
\end{array}\right) .
\end{aligned}
$$

Note que

$$
|B| \geqslant\left(\frac{2(k-\ell)-1}{2(k-\ell)} t\right)\left(1-\varepsilon_{0}\right) \frac{n}{t}-t \geqslant\left(\frac{2(k-\ell)-1}{2(k-\ell)}-\varepsilon_{0}\right) n .
$$

Dessa forma, adicionando no máximo $\varepsilon_{0} n$ vértices de $V \backslash B$ em $B$, obtemos um conjunto $B^{\prime}$ com $\left|B^{\prime}\right|=\left\lfloor\frac{2(k-\ell)-1}{2(k-\ell)} n\right\rfloor$ tal que

$$
e_{\mathcal{H}}\left(B^{\prime}\right) \leqslant e_{\mathcal{H}}(B)+\varepsilon_{0} n\left(\begin{array}{c}
n \\
k-1
\end{array}\right) \leqslant 6 C^{\prime} \gamma\left(\begin{array}{l}
n \\
k
\end{array}\right)=C \gamma\left(\begin{array}{l}
n \\
k
\end{array}\right)
$$

de onde concluímos que $\mathcal{H}$ é $(\ell, C \gamma)$-extremal.

\subsection{Prova do Teorema Principal de Hipergrafos}

Prova do Teorema 4.4. Fixe $0<\xi<1$ e inteiros $k \geqslant 4$ e $1 \leqslant \ell<k / 2$. Sejam $C$ e $\gamma_{0}$ as constantes obtidas pelo Lema de Empacotamento em Caminhos (Lema 4.16) para $k$ e $\ell$. Fixe $\gamma<\gamma_{0}$ uma constante suficientemente pequena. Seja $\varepsilon$ obtido pelo Lema de Absorção (Lema 4.8) para $\gamma, \zeta=\gamma$, $k$ e $\ell$. Seguindo a quantificação do Lema de Empacotamento em Caminhos (Lema 4.16) para $\alpha=\varepsilon / 2$ e $3 \gamma$, obtemos um inteiro $s$. Aplicamos o Lema do Reservatório (Lema 4.7) para um $\eta_{\text {res }}=\eta_{\text {res }}(\gamma, \varepsilon$ ) suficientemente pequeno e $\varepsilon_{\text {res }}=\varepsilon_{\text {res }}(\gamma, \varepsilon), k$ e $s$. Sejam $n \in(k-\ell) \mathbb{N}$ suficientemente grande e $\mathcal{H}$ um hipergrafo $k$-uniforme de ordem $n$.

Suponha que $\mathcal{H}$ não é $(\ell, \xi)$-extremal e

$$
\delta_{k-2}(\mathcal{H}) \geqslant\left(\frac{4(k-\ell)-1}{4(k-\ell)^{2}}-\gamma\right)\left(\begin{array}{l}
n \\
2
\end{array}\right)
$$

Seja $\mathcal{A}$ o $\ell$-caminho $\varepsilon$-absorvedor, com extremidades $X_{0}$ e $Y_{0}$, obtido pelo Lema de Absorção (Lema 4.8). Denote por $V^{\prime}=(V \backslash V(\mathcal{A})) \cup\left\{X_{0}, Y_{0}\right\}$ e por $\mathcal{H}^{\prime}=\mathcal{H}\left[V^{\prime}\right]$ o subhipergrafo de $\mathcal{H}$ induzido por $V^{\prime}$. Note que

$$
\delta_{k-2}\left(\mathcal{H}^{\prime}\right) \geqslant\left(\frac{4(k-\ell)-1}{4(k-\ell)^{2}}-2 \gamma\right)\left(\begin{array}{l}
n \\
2
\end{array}\right) .
$$

Para todo $j \leqslant s$, o Lema do Reservatório (Lema 4.7) garante a existência de um conjunto $R \subset V^{\prime} \operatorname{com}|R| \leqslant \varepsilon_{\text {res }} n \leqslant \gamma n$ tal que toda família $\left\{X_{i}, Y_{i}\right\}_{i \in[j]}$ de pares mutuamente disjuntos de $\ell$ vértices pode ser conectada através de caminhos que contêm apenas vértices de $\bigcup_{i \in[j]}\left(X_{i} \cup Y_{i}\right) \cup R$.

Denote por $V^{\prime \prime}=V \backslash(V(\mathcal{A}) \cup R)$ e por $\mathcal{H}^{\prime \prime}=\mathcal{H}\left[V^{\prime \prime}\right]$ o subhipergrafo de $\mathcal{H}$ induzido por $V^{\prime \prime}$. Dessa forma, temos

$$
\delta_{k-2}\left(\mathcal{H}^{\prime \prime}\right) \geqslant\left(\frac{4(k-\ell)-1}{4(k-\ell)^{2}}-3 \gamma\right)\left(\begin{array}{l}
n \\
2
\end{array}\right) .
$$

Pelo Lema de Empacotamento em Caminhos (Lema 4.16) em $\mathcal{H}^{\prime \prime}$ obtemos uma família de $\ell$ - 
caminhos disjuntos com no máximo $s$ elementos e que cobre todos os vértices exceto por no máximo $\alpha\left|V^{\prime \prime}\right| \leqslant \alpha n$ vértices de $\mathcal{H}^{\prime \prime}$, ou $\mathcal{H}^{\prime \prime}$ é $(\ell, C \gamma)$-extremal. Seja $n^{\prime \prime}=\left|V^{\prime \prime}\right|$. Suponha por contradição que $\mathcal{H}^{\prime \prime}$ é $(\ell, C \gamma)$-extremal. Então existe um conjunto $B \subset V^{\prime \prime}$ tal que $|B|=\left\lfloor\frac{2(k-\ell)-1}{2(k-\ell)} n^{\prime \prime}\right\rfloor$ e $e(B) \leqslant C \gamma\left(n^{\prime \prime}\right)^{k}$. Adicionando no máximo $n-n^{\prime \prime} \leqslant 2 \gamma n$ vértices de $V \backslash B$ em $B$, obtemos um conjunto de vértices $B^{\prime} \subset V$ tal que $\left|B^{\prime}\right|=\left\lfloor\frac{2(k-\ell)-1}{2(k-\ell)} n\right\rfloor \mathrm{e}$

$$
e\left(B^{\prime}\right) \leqslant C \gamma\left(n^{\prime \prime}\right)^{k}+2 \gamma n\left(\begin{array}{l}
n-1 \\
k-1
\end{array}\right) \leqslant \xi n^{k}
$$

uma contradição com o fato de que $\mathcal{H}$ não é $(\ell, \xi)$-extremal. Portanto, podemos assumir que existe uma família de $\ell$-caminhos disjuntos $\mathcal{P}_{1}, \ldots, \mathcal{P}_{j}$ com $j \leqslant s$ no qual cobre todos os vértices de $\mathcal{H}$ exceto por no máximo uma quantidade $\alpha\left|V^{\prime \prime}\right| \leqslant \alpha n$ de vértices de $\mathcal{H}^{\prime \prime}$. Denote por $T$ o conjunto de vértices não cobertos de $V^{\prime \prime}$.

Para todo $i \in[j]$, denotamos as extremidades de $\mathcal{P}_{i}$ por $X_{i}$ e $Y_{i}$ e seja $Y_{j+1}=Y_{0}$. Usando o Lema do Reservatório (Lema 4.7) para conectar a família $\left(X_{i}, Y_{i+1}\right)_{0 \leqslant i \leqslant j}$, conectamos os $\ell$-caminhos $\mathcal{A}, \mathcal{P}_{1}, \ldots, \mathcal{P}_{j}$ em um $\ell$-circuito $\varepsilon$-gerador $\mathcal{C} \subset \mathcal{H}$.

Seja $U=V \backslash V(\mathcal{C})$ o conjunto de vértices que não estão contidos em $\mathcal{C}$. Como $U \subset R \cup T$, temos que $|U| \leqslant\left(\varepsilon_{\text {res }}+\alpha\right) n \leqslant \varepsilon n$. Mais ainda, como $\mathcal{C}$ é um $\ell$-circuito e $n \in(k-\ell) \mathbb{N}$, temos $|U| \in(k-\ell) \mathbb{N}$. Portanto, podemos usar a propriedade de absorção de $\mathcal{A}$ para substituir $\mathcal{A}$ em $\mathcal{C}$ por um $\ell$-caminho $\mathcal{Q}$ com as mesmas extremidades de $\mathcal{A}$ e obtendo dessa forma o circuito Hamiltoniano desejado em $\mathcal{H}$. 


\section{Capítulo 5}

\section{Considerações Finais}

Neste capítulo, apresentamos algumas direções a seguir acerca dos problemas estudados nesta tese. No primeiro resultado desta tese, explicitamos o valor de empacotamento para a família de permutações em camadas composta por $k$ camadas e uma anticamada de tamanho $a \geqslant 2$ que satisfaz $2^{a}-a-1 \geqslant k$. Como discutido no Capítulo 3 , ainda existe um vasta família de permutações para os quais o Problema 3.1 continua em aberto. Acreditamos que a adaptação feita ao Algoritmo de Price ainda pode ser utilizada para abordar outras famílias de permutações. Dessa forma, listamos abaixo algumas famílias de permutações possíveis de serem estudadas utilizando essa abordagem.

i) Permutações do tipo $(\widehat{a}, \ell, \widehat{a}), \operatorname{com} a, \ell \geqslant 2$;

ii) Permutações do tipo $(k, k, 1, k, k)$, com $k \geqslant 2$;

iii) Permutações 21354 e 1324 .

Por outro lado, acreditamos que uma abordagem do Teorema 3.2 de forma não assintótica nos forneceria uma intuição melhor sobre a natureza do problema restrito a permutações em camadas.

No segundo resultado desta tese, provamos que a Conjectura de Myers (Conjectura 3.6) é verdadeira para o caso em que a permutação reversa é maior que a permutação identidade e o espaço é restrito ao conjunto de permutações em camadas. Para melhorar esse resultado, o passo seguinte mais natural consiste em expandir o espaço que estamos considerando. Dessa forma, um caminho natural é tentar expandir o espaço de busca para o conjunto de permutações cujas inversas são permutações em camadas. Para essa abordagem, uma vez que o Algoritmo de Price considera apenas permutações em camadas, precisamos encontrar uma forma de estender as ferramentas desenvolvidas por Price para esse conjunto. Assim, apresentamos a seguinte conjectura.

Conjectura 5.1. Para todo $k, \ell \leqslant 2$ temos que

$$
\lim _{n \rightarrow \infty} \min \left\{p\left(\operatorname{Id}_{\ell}+\operatorname{Rev}_{k}, \sigma\right): \sigma \in \mathfrak{S}_{n}^{L} \cup \mathfrak{S}_{n}^{R}\right\}=\min \left\{\frac{1}{(\ell-1)^{k-1}}, \frac{1}{(k-1)^{\ell-1}}\right\}
$$

Note que a Conjectura 5.1 acima é similar à Conjectura de Myers.

Dados inteiros positivos $k$ e $\ell$ e $\gamma>0$, provamos que se $1 \leqslant \ell \leqslant k / 2$ e $k \geqslant 4$ então todo hipergrafo $k$-unifome $\mathcal{H}$ suficientemente grande que satisfaz

$$
\delta_{k-2}(\mathcal{H}) \geqslant\left(\frac{4(k-\ell)^{2}-1}{4(k-\ell)}+\gamma\right)\left(\begin{array}{c}
|V(\mathcal{H})| \\
2
\end{array}\right)
$$


e $|V(\mathcal{H})| \in(k-\ell) \mathbb{N}$ possui um $\ell$-circuito Hamiltoniano. Existem três direções possíveis de atacar problemas semelhantes.

A primeira direção consiste em realizar um trabalho similar ao realizado por Han e Zhao [11] e fazer a versão exata deste resultado, i.e., eliminar $\gamma$ do resultado acima. Para isso, uma forma possível é utilizar as técnicas desenvolvidas em [11] que provam a versão exata para $k=3$ e $\ell=1$. De fato, o nosso resultado já foi escrito dentro do contexto das técnicas utilizadas por Han e Zhao [11].

A segunda direção seria tentar eliminar a restrição sobre $\ell$ no Teorema 4.3, i.e., mostrar que o teorema é válido para todo $1 \leqslant \ell<k$. Para isso, estudaremos o trabalho realizado por Kühn, Mycroft e Osthus [17] no qual generalizaram o Teorema 4.1. A priore observa-se que a estrutura utilizada para mostrar que a cota inferior para o grau mínimo difere da apresentada neste trabalho para quando $k / 2<\ell<k$.

A terceira direção consiste em verificar se é possível adaptar as técnicas estudadas neste trabalho e mostrar que se um hipergrafo $k$-uniforme $\mathcal{H}$ satisfaz

$$
\delta_{k-3}(\mathcal{H}) \geqslant\left(\frac{12(k-\ell)^{2}-6(k-\ell)+1}{8(k-\ell)^{3}}+\gamma\right)\left(\begin{array}{l}
n \\
3
\end{array}\right)
$$

e $|V(\mathcal{H})| \in(k-\ell) \mathbb{N}$ então $\mathcal{H}$ possui um $\ell$-circuito Hamiltoniano. A condição de grau mínimo apresentada acima foi baseada na estrutura extremal $\mathcal{H}_{k, \ell}$ discutida no início do Capítulo 4. Se for possível provar esse resultado, estaríamos caminhando na direção de mostrar que de fato o hipergrafo $\mathcal{H}_{k, \ell}$ fornece a cota justa de $j$-grau mínimo, com $1 \leqslant j \leqslant k-1$, que um hipergrafo $k$-uniforme $\mathcal{H}$ deve satisfazer de forma a garantir a existência de $\ell$-circuito Hamiltoniano para todo $1 \leqslant \ell<k / 2$. 


\section{Referências Bibliográficas}

[1] Michael H. Albert, Mike D. Atkinson, Chris C. Handley, Derek A. Holton, and Walter Stromquist. On packing densities of permutations. Electron. J. Combin., 9(1):Research Paper 5, 20, 2002 .

[2] József Balogh, Ping Hu, Bernard Lidický, Oleg Pikhurko, Balázs Udvari, and Jan Volec. Minimum number of monotone subsequences of length 4 in permutations. Combin. Probab. Comput., 24(4):658-679, 2015.

[3] Josefran Oliveira Bastos and Leonardo Nagami Coregliano. Packing densities of layered permutations and the minimum number of monotone sequences in layered permutations. submitted.

[4] Josefran Oliveira Bastos, Guilherme Oliveira Mota, Mathias Schacht, Jakob Schnitzer, and Fabian Schulenburg. Loose hamiltonian cycles forced by large $(k-2)$-degree - approximate version -. submitted.

[5] Enno Buß, Hiêp Hàn, and Mathias Schacht. Minimum vertex degree conditions for loose Hamilton cycles in 3-uniform hypergraphs. J. Combin. Theory Ser. B, 103(6):658-678, 2013.

[6] Fan R. K. Chung. Regularity lemmas for hypergraphs and quasi-randomness. Random Structures Algorithms, 2(2):241-252, 1991.

[7] Gabriel A. Dirac. Some theorems on abstract graphs. Proc. London Math. Soc. (3), 2:69-81, 1952.

[8] Paul Erdős and George Szekeres. A combinatorial problem in geometry. Compositio Math., 2:463-470, 1935.

[9] Hiểp Hàn and Mathias Schacht. Dirac-type results for loose Hamilton cycles in uniform hypergraphs. J. Combin. Theory Ser. B, 100(3):332-346, 2010.

[10] Jie Han and Yi Zhao. Minimum codegree threshold for Hamilton $\ell$-cycles in $k$-uniform hypergraphs. J. Combin. Theory Ser. A, 132:194-223, 2015.

[11] Jie Han and Yi Zhao. Minimum vertex degree threshold for loose Hamilton cycles in 3-uniform hypergraphs. J. Combin. Theory Ser. B, 114:70-96, 2015.

[12] Peter A. Hästö. The packing density of other layered permutations. Electron. J. Combin., 9(2):Research paper 1, 16, 2002/03. Permutation patterns (Otago, 2003). 
[13] Richard M. Karp. Reducibility among combinatorial problems. In Complexity of computer computations (Proc. Sympos., IBM Thomas J. Watson Res. Center, Yorktown Heights, N.Y., 1972), pages 85-103. Plenum, New York, 1972.

[14] Gyula Y. Katona and Hal A. Kierstead. Hamiltonian chains in hypergraphs. J. Graph Theory, 30(3):205-212, 1999.

[15] Peter Keevash, Daniela Kühn, Richard Mycroft, and Deryk Osthus. Loose Hamilton cycles in hypergraphs. Discrete Math., 311(7):544-559, 2011.

[16] Dénes König. Theorie der endlichen und unendlichen Graphen. Kombinatorische Topologie der Streckenkomplexe. Chelsea Publishing Co., New York, N. Y., 1950.

[17] Daniela Kühn, Richard Mycroft, and Deryk Osthus. Hamilton $\ell$-cycles in uniform hypergraphs. J. Combin. Theory Ser. A, 117(7):910-927, 2010.

[18] Daniela Kühn and Deryk Osthus. Loose Hamilton cycles in 3-uniform hypergraphs of high minimum degree. J. Combin. Theory Ser. B, 96(6):767-821, 2006.

[19] Joseph Samuel Myers. The minimum number of monotone subsequences. Electron. J. Combin., 9(2):Research paper 4, 17 pp. (electronic), 2002/03. Permutation patterns (Otago, 2003).

[20] Oystein Ore. Note on Hamilton circuits. Amer. Math. Monthly, 67:55, 1960.

[21] Alkes L. Price. Packing densities of layered patterns. PhD thesis, University of Pennsylvania, Philadelphia, 1997.

[22] Alexander A. Razborov. Flag algebras. J. Symbolic Logic, 72(4):1239-1282, 2007.

[23] Vojtěch Rödl, Andrzej Ruciński, and Endre Szemerédi. A Dirac-type theorem for 3-uniform hypergraphs. Combin. Probab. Comput., 15(1-2):229-251, 2006.

[24] Vojtěch Rödl, Andrzej Ruciński, and Endre Szemerédi. An approximate Dirac-type theorem for $k$-uniform hypergraphs. Combinatorica, 28(2):229-260, 2008.

[25] Wojciech Samotij and Benny Sudakov. On the number of monotone sequences. arXiv preprint arXiv:1405.1623, 2014.

[26] Endre Szemerédi. Regular partitions of graphs. In Problèmes combinatoires et théorie des graphes (Colloq. Internat. CNRS, Univ. Orsay, Orsay, 1976), volume 260 of Colloq. Internat. CNRS, pages 399-401. CNRS, Paris, 1978.

[27] Dan Warren. Optimal packing behavior of some 2-block patterns. Ann. Comb., 8(3):355-367, 2004. 